\title{
Dependence of magnetic field generation by thermal convection on the rotation rate: a case study
}

\author{
R. Chertovskiha, ${ }^{\mathrm{a}, \mathrm{b}, *}$, S.M.A. Gama ${ }^{\mathrm{a}}$, O. Podvigina ${ }^{\mathrm{b}, \mathrm{c}}$, V. Zheligovsky ${ }^{\mathrm{b}, \mathrm{c}}$ \\ ${ }^{a}$ University of Porto, Faculty of Sciences, Department of Applied Mathematics, \\ Rua Campo Alegre 687, 4169-007 Porto, Portugal \\ ${ }^{b}$ International Institute of Earthquake Prediction Theory and Mathematical Geophysics, \\ 84/32 Profsoyuznaya St, 117997 Moscow, Russian Federation \\ ${ }^{c}$ Observatoire de la Côte d'Azur, BP 4229, 06304 Nice Cedex 4, France
}

\begin{abstract}
Dependence of magnetic field generation on the rotation rate is explored by direct numerical simulation of magnetohydrodynamic convective attractors in a plane layer of conducting fluid with square periodicity cells for the Taylor number varied from zero to 2000, for which the convective fluid motion halts (other parameters of the system are fixed). We observe 5 types of hydrodynamic (amagnetic) attractors: two families of two-dimensional (i.e. depending on two spatial variables) rolls parallel to sides of periodicity boxes of different widths and parallel to the diagonal, travelling waves and three-dimensional "wavy" rolls. All types of attractors, except for one family of rolls, are capable of kinematic magnetic field generation. We have found 21 distinct nonlinear convective MHD attractors (13 steady states and 8 periodic regimes) and identified bifurcations in which they emerge. In addition, we have observed a family of periodic, twofrequency quasiperiodic and chaotic regimes, as well as an incomplete Feigenbaum period doubling sequence of bifurcations of a torus followed by a chaotic regime and subsequently by a torus with $1 / 3$ of the cascade frequency. The system is highly symmetric. We have found two novel global bifurcations reminiscent of the SNIC bifurcation, which are only possible in the presence of symmetries. The universally accepted paradigm, whereby an increase of the rotation rate below a certain level is beneficial for magnetic field generation, while a further increase inhibits it (and halts the motion of fluid on continuing the increase) remains unaltered, but we demonstrate that this "large-scale" picture lacks many significant details.
\end{abstract}

Keywords: Rayleigh-Bénard convection, convection in rotating fluid, kinematic dynamo, nonlinear magnetohydrodynamic regimes, bifurcations

PACS: 47.20.Bp, 47.20.Ky, 91.25.Cw

\section{Introduction}

Magnetic field of stars, planets and other astrophysical objects is often attributed to the motion of electrically conducting melted substance in their interior (Parker, 1979; Priest, 1984; Soward et al., 2005; Hughes et al., 2007; Dormy and Soward 2007), which is usually sustained by compositional and thermal convection. Convective flows in a plane layer, ranging from very simple (Matthews, 1999) to turbulent (Meneguzzi and Pouquet, 1989; Cattaneo et al. 2003) ones, are capable of magnetic field generation. In simulations of the dynamo in the Earth's liquid core (Glatzmaier and Roberts, 1995; Roberts and Glatzmaier, 2000), a magnetic field of the approximately correct strength was produced, which had the dipole structure and exhibited reversals similar to the natural ones. Dynamos in spherical shells were also simulated by Grote and Busse (2001), Ishinara and Kida (2002), Takahashi and Matsushima (2005) and other authors.

\footnotetext{
* Corresponding author

Email addresses: roman@fc.up.pt, tel.: +351 220100 858, fax.: +351 220402209 (R. Chertovskih), smgama@fc.up.pt (S.M.A. Gama), olgap@mitp.ru (O. Podvigina), vlad@mitp.ru (V. Zheligovsky)
} 
Magnetohydrodynamic thermal convection is characterised in the dimensionless form by the Rayleigh number, $R$ (indicating the magnitude of thermal buoyancy forces); the Prandtl number, $P$ (the ratio of kinematic viscosity to thermal diffusivity), the magnetic Prandtl number, $P_{m}$ (the ratio of kinematic viscosity to magnetic diffusivity), and the Taylor number, $\mathrm{Ta}$ (measuring the speed of rotation). Usually no-slip or stress-free boundary conditions for the flow velocity and perfectly conducting or insulating boundaries for magnetic field are considered.

How magnetic field generation by convection depends on parameter values, is not yet explored in full. Large Rayleigh numbers are beneficial for generation (the critical $P_{m}$ decreases monotonically with the increasing $R$ ) in spherical shells (Busse, 2000). Podvigina (2006) found a similar dependence in plane layer dynamos on increasing $R$ over the critical value, but for $R$ over a certain threshold the behaviour of the critical $P_{m}$ ceased to be monotonic; Podvigina (2008) also examined the influence of the Prandtl number.

Rotation, which is a common feature of the majority of astrophysical bodies, can also assist magnetic field generation. Rotation of the Earth is relatively rapid; it is believed, that geodynamo operates in the outer core in the magnetostrophic regime, in which the strength of the Coriolis force is comparable to that of other primary forces - the Lorentz force, pressure and buoyancy. Rotation is also an important factor in physics of the Solar tachocline (Christensen-Dalsgaard and Thompson, 2007), where apparently the solar dynamo is located. Differential rotation gives rise to $\omega$-effect dynamos (Moffatt, 1978). Although they are slow, as opposed to fast ones, which are believed to operate in stars, this mechanism is regarded as a key element of the dynamo of the Sun (Tobias and Weiss, 2007). Boundary layers and shear flows developing in rapidly rotating fluids are the structures controlling the dynamics of fluid and planetary dynamo processes (Busse et al., 2007). Therefore, how the processes of generation are affected by the rate of rotation is an interesting question for astrophysical applications.

Dependence of magnetic field generation on the rate of rotation was explored in a number of papers. Meneguzzi and Pouquet (1989) observed that rotation could be beneficial for nonlinear dynamos - the critical magnetic Reynolds number decreased and the ratio of magnetic to kinetic energies significantly increased, when rotation was on. By contrast, Cattaneo and Hughes (2006) found that rotation was not a significant factor: they reported "similar growth rates and similar saturation levels" in rotating and nonrotating systems. Only several runs were presented in each of the two papers, and hence the observations were inconclusive. Whilst turbulent convective nonlinear dynamos do not require rotation, near the onset of convection in a plane layer rotation is essential for both kinematic (Matthews, 1999) and nonlinear dynamo action (Demircan and Seehafer, 2002) - in both regimes considered by these authors dynamos failed in the absence of rotation.

Our paper is devoted to investigation of the dependence of magnetic field generation on Ta. A fluid heated from below in a plane horizontal layer rotating about the vertical axis is considered in the Boussinesq approximation, whereby the buoyancy depends linearly on temperature, density variation is neglected in the mass conservation equation and the flow is incompressible. Perfectly electrically conducting stress-free horizontal boundaries of the layer are held at constant temperatures; periodicity in horizontal directions with the same period $L$ (measured in the units of the layer depth) is assumed.

We fix all parameter values except for the Taylor number and investigate numerically attractors of the system for $T a$ increasing from zero, and bifurcations delimiting branches of the attractors. Along the branches we trace average magnetic, $E_{m}$, and kinetic, $E_{k}$, energies, as well as their ratio, $E_{m} / E_{k}$, in saturated regimes; the latter quantity is a measure of dynamo efficiency of prime concern in astrophysics (although we are clearly not in the astrophysical range of parameter values). The system is also of interest from the point of view of equivariant bifurcation theory, because it has a large symmetry group.

Which parameter values are optimal for such an investigation? Naturally, we need to employ the values, for which the structure of attractors of the dynamical system and the geometry of their branches in the parameter space are relatively simple. Behaviour of the dynamical system, which one encounters in the geoand astrophysical environments or in experimental dynamos, results from a large number of bifurcations of the trivial steady state, and it is prohibitively complex to serve as a starting point for such a study. These difficult cases can be approached later by continuation in parameters starting at the regimes determined in the initial study. Also, in the initial investigation we cannot employ relatively small values of $P_{m}$, because for them only vigorous turbulent flows would yield magnetic dynamos: Many hydrodynamic bifurcations 
would be required to bring the convective MHD system into the turbulent state and, consequently, for such parameter values the system will also be prohibitively complex. By contrast, for relatively large $P_{m}$ already laminar convective flows of simple structure can act as dynamos; moreover, if $P_{m}$ is not too large, magnetic field will not be too strong to enable the Lorentz force to destabilise the convective flows. This suits ideally our purposes.

Following these considerations, we employed the same values as in Podvigina (2006): $P=1, P_{m}=8$, $R=2300$ and $L=2 \sqrt{2}$. The Rayleigh number is not far from the critical value for the onset of convection; hence, convective attractors have a simple roll structure, which simplifies a detailed investigation of the attractors and bifurcations. The $P_{m}$ value is not far from its critical values for the kinematic dynamo problem for convective attractors, hence flows in convective MHD attractors (when dynamos operate) are qualitatively similar to flows in non-magnetic ones. (Note, that in steady MHD states flows can be reconstructed from the structure of the generated magnetic field, see Zheligovsky, 2009a.) The assumed aspect ratio, $L$, is equal to the spatial period (measured in the units of the layer width) of the hydrodynamic mode becoming unstable the first when the onset of convective motion occurs in a non-rotating layer on increasing the Rayleigh number.

Although these values are chosen on the basis of mathematical convenience, they are not unphysical: $P_{m}$ are of the order of unity in accretion disks (von Rekowski et al., 2001); magnetic Prandtl numbers $P_{m}$ in excess of unity are typical for intergalactic and interstellar gases (Shukurov and Sokoloff, 2008; see also Table 1 in Brandenburg and Subramanian, 2005). A plasma experiment for the study of astrophysical dynamos, where $3 \cdot 10^{-4} \leq P_{m} \leq 5$, was proposed by Spence et al.(2009). We also note that typically galaxies are thin disks (see Ruzmaikin et al., 1988), and hence some features of the problem at hand (an infinite plane layer with stress-free boundaries) are appropriate to model galactic dynamos. We stress nevertheless, that we solve the problem in an abstract setting and do not pretend to simulate any specific physical system.

\section{Statement of the problem}

The system is governed by the Navier-Stokes equation

$$
\frac{\partial \mathbf{v}}{\partial t}=\mathbf{v} \times(\nabla \times \mathbf{v})+P \Delta \mathbf{v}+P R \theta \mathbf{e}_{z}+P \tau \mathbf{v} \times \mathbf{e}_{z}-\nabla p-\mathbf{b} \times(\nabla \times \mathbf{b}),
$$

the magnetic induction equation

$$
\frac{\partial \mathbf{b}}{\partial t}=\nabla \times(\mathbf{v} \times \mathbf{b})+P P_{m}^{-1} \Delta \mathbf{b}
$$

for solenoidal fields

$$
\nabla \cdot \mathbf{v}=0, \nabla \cdot \mathbf{b}=0
$$

and the heat transfer equation

$$
\frac{\partial \theta}{\partial t}=-(\mathbf{v} \cdot \nabla) \theta+v_{z}+\Delta \theta
$$

Here $\mathbf{v}$ denotes the flow velocity, $\mathbf{b}$ the magnetic field, $\theta$ the difference between the temperature of fluid and the linear temperature profile, and $\tau=\sqrt{T a}$ is twice the angular speed of rotation of the fluid.

The following boundary conditions on the horizontal boundaries:

$$
\begin{gathered}
\frac{\partial v_{x}}{\partial z}=\frac{\partial v_{y}}{\partial z}=v_{z}=0, \quad \theta=0 \quad \text { at } z=0,1 \\
\frac{\partial b_{x}}{\partial z}=\frac{\partial b_{y}}{\partial z}=b_{z}=0 \quad \text { at } z=0,1
\end{gathered}
$$

and periodicity in horizontal directions with the same period

$$
\begin{gathered}
\mathbf{v}(x, y, z)=\mathbf{v}(x+m L, y+n L, z), \quad \theta(x, y, z)=\theta(x+m L, y+n L, z), \\
\mathbf{b}(x, y, z)=\mathbf{b}(x+m L, y+n L, z) \\
\forall m, n \in \mathbf{Z}
\end{gathered}
$$

are assumed. 
The equations are solved numerically by the standard pseudospectral methods (Boyd, 2001; Peyret, 2002). Fields are represented as Fourier series satisfying the boundary conditions (2):

$$
\begin{gathered}
\mathbf{v}=\sum_{\mathbf{n}}\left(\begin{array}{c}
\hat{v}_{\mathbf{n}}^{x} \cos \left(\pi n_{3} z\right) \\
\hat{v}_{\mathbf{n}}^{y} \cos \left(\pi n_{3} z\right) \\
\hat{v}_{\mathbf{n}}^{z} \sin \left(\pi n_{3} z\right)
\end{array}\right) \mathrm{e}^{\frac{2 \pi \mathrm{i}}{L}\left(n_{1} x+n_{2} y\right)}, \\
\mathbf{b}=\sum_{\mathbf{n}}\left(\begin{array}{c}
\hat{b}_{\mathbf{n}}^{x} \cos \left(\pi n_{3} z\right) \\
\hat{b}_{\mathbf{n}}^{y} \cos \left(\pi n_{3} z\right) \\
\hat{b}_{\mathbf{n}}^{z} \sin \left(\pi n_{3} z\right)
\end{array}\right) \mathrm{e}^{\frac{2 \pi \mathrm{i}}{L}\left(n_{1} x+n_{2} y\right)}, \\
\theta=\sum_{\mathbf{n}} \hat{\theta}_{\mathbf{n}} \sin \left(\pi n_{3} z\right) \mathrm{e}^{\frac{2 \pi \mathrm{i}}{L}\left(n_{1} x+n_{2} y\right)} .
\end{gathered}
$$

The resolution of $31 \times 31 \times 17$ Fourier harmonics has been employed for computation of hydrodynamic convective attractors, and $63 \times 63 \times 33$ in simulations of kinematic dynamos (without dealiasing in both cases). These simulations have been checked against runs with the double resolution without dealiasing, and computations of kinematic magnetic modes also against runs with the resolution of $41 \times 41 \times 21$ harmonics with dealiasing. We have been employing the standard method of dealiasing in computation of all products in (1) (requiring to evaluate fields on a uniform $64 \times 64 \times 33$ mesh in the physical space in order to compute $41 \times 41 \times 21$ Fourier harmonics of the products).

Simulation of nonlinear hydromagnetic convective regimes has been performed with the resolution of $41 \times 41 \times 21$ Fourier harmonics with dealiasing. We note that computation with a coarser resolution of $31 \times 31 \times 16$ Fourier harmonics without dealiasing in earlier MHD convection simulations by Gertsenshtein et al. $(2007,2008)$ yielded a wrong classification of some regimes (a typical error consisted of obtaining a periodic regime instead of a convective MHD steady state). Although from the conservative point of view it thus may be also unsafe to employ the resolution of $41 \times 41 \times 21$ harmonics, the huge amount of runs has forced us to use it in the main bulk of computations. For this resolution magnetic energy spectrum decreases 1 by at least four orders of magnitude and the flow and temperature energy spectra decrease by about nine orders of magnitude. Some computations of nonlinear hydromagnetic regimes (including at least one attractor on each MHD branch) have been checked against runs with the resolution of $127 \times 127 \times 65$ Fourier harmonics without dealiasing, the results remaining visibly unaffected (the energies being reproduced with the accuracy better than $0.001 \%$ ).

By $E_{k}$ and $E_{m}$ we denote kinetic and magnetic energies, respectively, averaged over the fluid layer:

$$
E_{k}=\frac{1}{L^{2}} \int_{0}^{1} \int_{0}^{L} \int_{0}^{L} \frac{|\mathbf{v}|^{2}}{2} d x d y d z, \quad E_{m}=\frac{1}{L^{2}} \int_{0}^{1} \int_{0}^{L} \int_{0}^{L} \frac{|\mathbf{b}|^{2}}{2} d x d y d z .
$$

For non-steady convective MHD regimes, time averaging is also performed.

Branches of attractors are traced by continuation in parameter: computations are done for initial conditions, which are a point (in the phase space) on the attractor for a "nearby" Ta. Typically the distances between such "nearby" $T a$ vary between 0.1 and 100. Also, for some Ta runs have been performed for "random" initial conditions, comprised of fields with pseudorandomly generated Fourier coefficients and an exponentially decaying spectrum, with either small $\left(\sim 10^{-6}\right)$, or large $\left(\sim 10^{2}-10^{4}\right)$ initial kinetic, magnetic and thermal energies, in order to check whether multiple attractors coexist for the value of the Taylor number under consideration. Bifurcations of steady states are located by solving the eigenvalue problem near the endpoints of the branches and extrapolating the eigenvalues or their real parts to zero.

\footnotetext{
${ }^{1}$ The ratio of energy in the spherical shell of width one in the Fourier space with the largest energy content to the energy in the last considered spherical shell is reported.
} 


\section{Symmetries}

The symmetries of the rotating hydromagnetic convective system under consideration constitute a subgroup of the group of symmetries of the system in the absence of rotation. We list them here for reader's convenience, using the notation of Podvigina (2006).

The symmetry group of the convective system (1) with the boundary conditions (2), (3) is $\mathbf{Z}_{4} \ltimes \mathbf{T}^{2} \times \mathbf{Z}_{2}$. The group $\mathbf{Z}_{4}$ consists of rotations

$$
\begin{aligned}
& s_{1}:(x, y, z) \mapsto(y,-x, z), \\
& s_{2}:(x, y, z) \mapsto(-x,-y, z), \\
& s_{3}:(x, y, z) \mapsto(-y, x, z)
\end{aligned}
$$

and the identity $s_{0}=e . \mathbf{T}_{x}$ and $\mathbf{T}_{y}$ are the groups of translations in the $x$ and $y$ directions, respectively:

$$
\begin{aligned}
& \gamma_{\alpha}^{x}:(x, y, z) \mapsto(x+\alpha, y, z), \\
& \gamma_{\alpha}^{y}:(x, y, z) \mapsto(x, y+\alpha, z)
\end{aligned}
$$

where $0 \leq \alpha<L\left(\gamma_{L}^{x}=\gamma_{L}^{y}=e\right) . \mathbf{T}_{x y}$ is the group of translations along the diagonal:

$$
\gamma_{\alpha}^{x y}:(x, y, z) \mapsto(x+\alpha, y+\alpha, z) .
$$

The group $\mathbf{Z}_{2}$ is generated by reflections about the horizontal midplane:

$$
r:(x, y, z) \mapsto(x, y, 1-z) .
$$

If magnetic field is present, the group of symmetries of the system is augmented by the symmetry reversing magnetic field

$$
q:(\mathbf{v}, \theta, \mathbf{b}) \mapsto(\mathbf{v}, \theta,-\mathbf{b}) .
$$

The symmetry about a vertical axis, $s_{2}$, and parity invariance about a centre (located on the midplane) which is a composition of $s_{2}$ and $r$, are of special interest, since in such MHD states the global $\alpha$-effect is zero: In the presence of the global $\alpha$-effect the system is inherently unstable to large-scale perturbations, while in its absence the instability, if present, develops on time scales of a higher order (see Zheligovsky, 2009b). Although we have not studied spatio-temporal symmetries of non-steady attractors in detail, we note that we have encountered periodic orbits possessing the symmetry about a vertical axis or parity invariance with a time shift (equal to a half of the temporal period). A spatio-temporal symmetry with a time shift differs from the respective spatial symmetry in that it relates fields at two time instances, separated by an interval whose length is equal to the time shift; for instance, the symmetry $s_{2}$ about a vertical axis through the point $\left(a_{x}, a_{y}, 0\right)$ with a time shift $T$ of a vector field $\mathbf{f}$ is defined by the conditions

$$
\begin{aligned}
& f_{x}\left(a_{x}-x, a_{y}-y, z, t\right)=-f_{x}\left(a_{x}+x, a_{y}+y, z, t+T\right), \\
& f_{y}\left(a_{x}-x, a_{y}-y, z, t\right)=-f_{y}\left(a_{x}+x, a_{y}+y, z, t+T\right), \\
& f_{z}\left(a_{x}-x, a_{y}-y, z, t\right)=f_{z}\left(a_{x}+x, a_{y}+y, z, t+T\right) .
\end{aligned}
$$

Note that compositions $s_{2} \gamma_{\alpha}^{d}$ and $s_{2} r \gamma_{\alpha}^{d}$ are the symmetry about a vertical axis and parity invariance, in which the axis (the centre, respectively) of symmetry is displaced in the direction $d$ by $-\alpha / 2$; changing the order of factors in these compositions to the opposite one results in the displacement of the axis and the centre, respectively, in the reverse direction. $s_{2}$ is used as a generic notation for the symmetry about a vertical axis with no specific axis indicated; consequently, we do not distinguish notationally $s_{2} \gamma_{\alpha}^{d}$ from $s_{2}$ (except for in Table 5). In what follows, for construction of figures displaying vector fields which possess the symmetry $s_{2}$ we employ a coordinate system with the origin lying on the axis of $s_{2}$. 


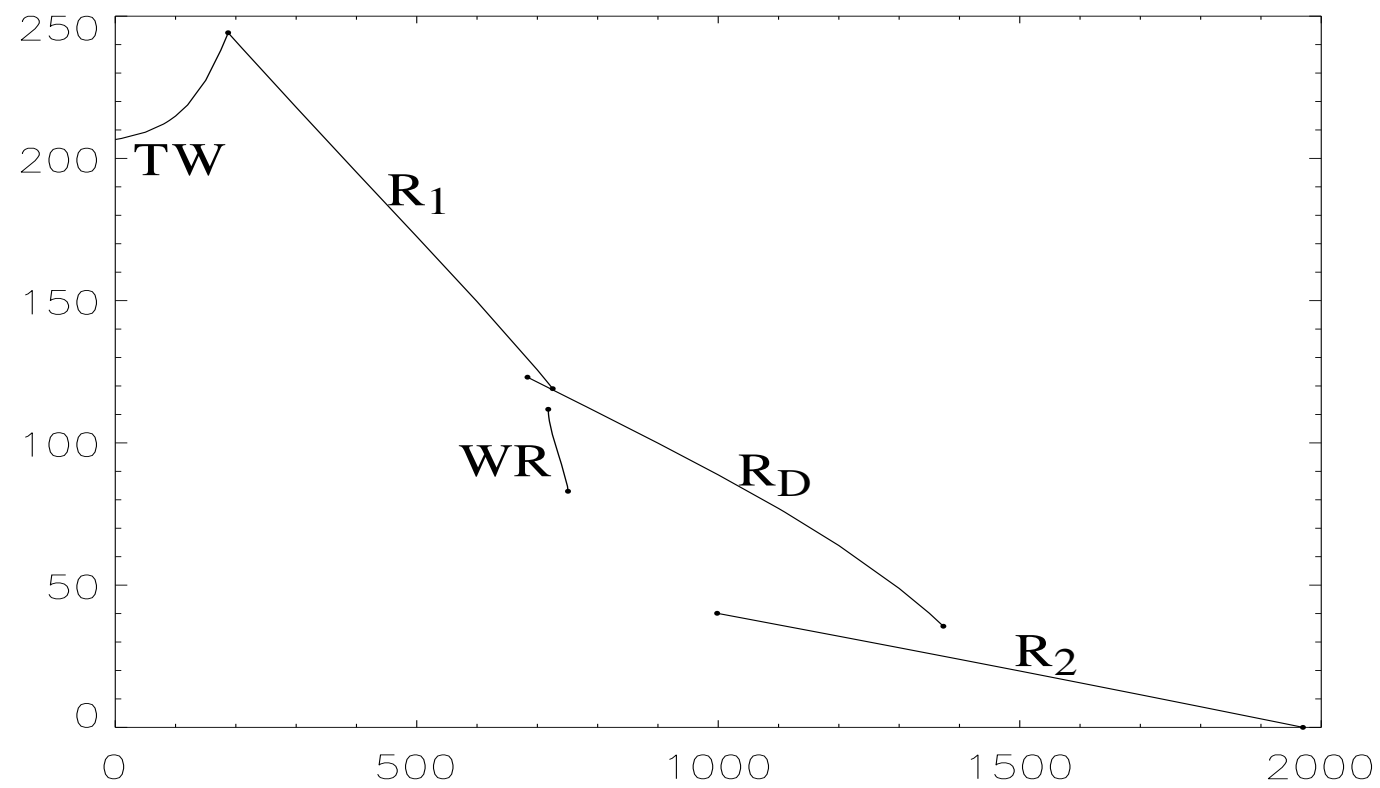

Figure 1: Kinetic energy (vertical axis) of hydrodynamic attractors for $0<T a \leq 2000$ (horizontal axis). Labelling of attractors is explained in Section 4 (see also Table 1). Note that $R_{1}$ does not bifurcate from $R_{D}$ : energies of the two flows at the point, where $R_{1}$ becomes unstable, differ by about 0.5 , which is not seen in the scale of the figure.

\section{Hydrodynamic convective attractors}

For a small Rayleigh number, i.e. a small temperature difference between the upper and lower boundaries, the fluid is not moving and heat is transported by thermal diffusion only. When $R$ exceeds the critical value (which increases with $T a$ ), a fluid motion sets in. For $P=1$ considered here, the instability is monotonic, the primary motion has the form of rolls. The wave number (in the horizontal direction) of the most unstable mode monotonically increases with $T a$. If periodicity in horizontal directions is imposed, the horizontal wave number must be compatible with the periodicity cell size and it can take only discrete values, and for an increasing $T a$ the wave numbers of the emerging rolls constitute a piecewise constant growing function. At very high rotation rates an array of very thin rolls emerges (Bassom and Zhang, 1994).

Five types of attractors of hydrodynamic convection (governed by the system $(1)-(3)$ for $\mathbf{b}=0$ ), that we have found in computations, are shown on the bifurcation diagram Fig. 1 (see also Table 11). Since the Rayleigh number is not far from the critical value for the onset of convection, the flows are of a simple spatial structure (see Fig. 2).

Three types of attractors are steady rolls of different spatial periods. In agreement with the linear stability theory, their wave numbers increase with $T a$ (remaining the same within each branch). The rolls obtained in computations are parallel to a side of the periodicity cell with either the same period as the periodicity cell size, or a half of it; or they are parallel to the diagonal of the cell. The branches are labelled $\mathrm{R}_{1}, \mathrm{R}_{2}$ and $\mathrm{R}_{\mathrm{D}}$, respectively.

In a non-rotating layer, bifurcations of rolls are well documented. However, these results cannot be directly applied to convection in a rotating layer, even if the rotation rate is small - this can only be done after unfolding of bifurcations of rolls is performed, i.e. after it is determined how the bifurcations alter, if small terms breaking the reflection symmetry are added. This is an interesting problem in its own right, but it is beyond the scope of the paper.

On increasing $R$, in a non-rotating layer rolls can bifurcate to travelling waves in a Hopf bifurcation for $P \lesssim 1$ (Getling, 1998). This bifurcation, with the $\mathbf{O}(2)$ symmetry group, was studied in detail in Golubitsky et al. (1988). In it, branches of standing and travelling waves emerge; if both branches bifurcate supercritically, one of them is stable. A travelling wave, TW, is time-periodic in a coordinate frame at 
Table 1: Attractors found numerically for hydrodynamic $(\mathbf{b}=0)$ convection. Column 2 presents the interval, where existence of branches of attractors is confirmed numerically, column 3 presents the symmetry group for which an attractor is pointwise invariant, column 4 generators of the group (for an appropriately chosen Cartesian coordinate system); if a group is a product of several subgroups, generators of the subgroups are separated by semicolons. Column 5 presents locations ( $T a$ ) and types of bifurcations in which a steady state becomes unstable ( $\mathrm{S}$ denotes a steady-state bifurcation, $\mathrm{H}$ a Hopf bifurcation), column 6 the dimension of the respective center eigenspace, column 7 the action of the symmetry group on the null eigenspace, and the last column elements of the group which act trivially. 1 denotes a trivial group of symmetries.

\begin{tabular}{|c|c|c|c|c|c|c|c|}
\hline Type & $\begin{array}{c}\text { Interval of } \\
\text { existence }(T a)\end{array}$ & $\begin{array}{c}\text { Symmetry } \\
\text { group }\end{array}$ & Generators & Bifurcation & D & Action & Kernel \\
\hline $\mathrm{TW}$ & {$[1,187]$} & $\mathbf{Z}_{2}$ & $r \gamma_{L / 2}^{x}$ & & & & \\
\hline $\mathrm{R}_{1}$ & {$[188,725]$} & $\mathbf{D}_{2} \ltimes \mathbf{T}$ & $r \gamma_{L / 2}^{x}, s_{2} ; \gamma^{y}$ & $187.19, \mathrm{H}$ & 4 & $\mathbf{O}(2)$ & $r \gamma_{L / 2}^{x}$ \\
& & & & $725.45, \mathrm{~S}$ & 2 & $\mathbf{O}(2)$ & $r \gamma_{L / 2}^{x}$ \\
\hline $\mathrm{WR}$ & {$[719,750]$} & $\mathbf{D}_{2}$ & $r \gamma_{L / 2}^{x}, s_{2}$ & $718.16, \mathrm{~S}$ & 1 & $\mathbf{1}$ & $r \gamma_{L / 2}^{x}, s_{2}$ \\
& & & & $750.82, \mathrm{~S}$ & 1 & $\mathbf{Z}_{2}$ & $s_{2} r \gamma_{L / 2}^{x}$ \\
\hline $\mathrm{R}_{\mathrm{D}}$ & {$[684,1373]$} & $\mathbf{D}_{2} \ltimes \mathbf{T}$ & $r \gamma_{L / 2}^{x}, s_{2} ; \gamma^{x y}$ & $683.64, \mathrm{~S}$ & 2 & $\mathbf{O}(2)$ & $r \gamma_{L / 2}^{x}$ \\
& & & & $1373.32, \mathrm{~S}$ & 2 & $\mathbf{O}(2)$ & $r \gamma_{L / 4}^{x,-y}$ \\
\hline $\mathrm{R}_{2}$ & {$[999,1969]$} & $\mathbf{D}_{4} \ltimes \mathbf{T}$ & $r \gamma_{L / 4}^{x}, s_{2} ; \gamma^{y}$ & $998.18, \mathrm{~S}$ & 2 & $\mathbf{O}(2)$ & $r \gamma_{L / 4}^{x y}$ \\
\hline
\end{tabular}

rest and it is steady in a frame moving with the speed of the pattern. In a non-rotating convection such bifurcation from $\mathrm{R}_{1}$ to a stable travelling wave takes place near $R \approx 1755$ (Podvigina, 2006). The instability causes a sinusoidal bending of rolls, the pattern travelling along the axis of a roll.

We also observe the TW: On decreasing $T a, \mathrm{R}_{1}$ loses stability and the stable travelling wave emerges in a similar supercritical Hopf bifurcation with the $\mathbf{O}(2)$ symmetry group. Due to rotation, no reflection symmetries are present, enabling the travelling wave to drift in the direction perpendicular to axes of rolls. All other bifurcations of rolls of the three types are subcritical pitchfork bifurcations, with no stable branches emerging. On increasing $T a$, the branch $\mathrm{R}_{2}$ terminates at $T a=1969.67$ (this number is in a good agreement with the value of the Taylor number, obtained from the Chandrasekhar's (1961) formula for the critical value of the Rayleigh number for the onset of convection in a layer with free boundaries) on the trivial steady state; no non-trivial convective regimes exist for higher rotation rates.

Another attractor, distinct from rolls, is wavy rolls, WR. In a non-rotating layer emergence of WR from $\mathrm{R}_{1}$ is a consequence of the $1: \sqrt{2}$ mode interaction (Podvigina and Ashwin, 2007). Similar arguments show that in a rotating layer WR can also bifurcate from rolls. On decreasing Ta, WR disappear in a saddle-node bifurcation - the branch turns back and becomes unstable. Apparently, it bifurcates from $\mathrm{R}_{1}$ at $T a=725.45$ : WR and $\mathrm{R}_{1}$ have similar shapes and close energy values, and by the equivariant branching lemma (Golubitsky et al., 1988) the action of the symmetry group of $\mathrm{R}_{1}$ (see Table 1) implies that a branch with the symmetries of WR bifurcates from $R_{1}$.

\section{Magnetic field generation}

We have investigated whether the flows that are convective hydrodynamic attractors are capable of kinematic magnetic field generation. For steady hydrodynamic states we have been computing (applying the algorithm of Zheligovsky, 1993a,b) dominant eigenvalues of the magnetic induction operator. For travelling waves, equation (2.b) in the co-moving reference frame yields a similar eigenvalue problem (see Podvigina, 2006).

All the hydrodynamic attractors have non-trivial symmetry groups and magnetic modes can be classified in the terms of the action of their symmetries. Symmetry groups of rolls are continuous, they include shifts along the axis of rolls, and bifurcating modes can have arbitrary periods in this direction. We restrict our attention to magnetic modes that have the periodicity of the hydrodynamic convective attractors. The computed growth rates are shown on Fig. 3, and symmetries of dominant modes are presented in Table 2 It 


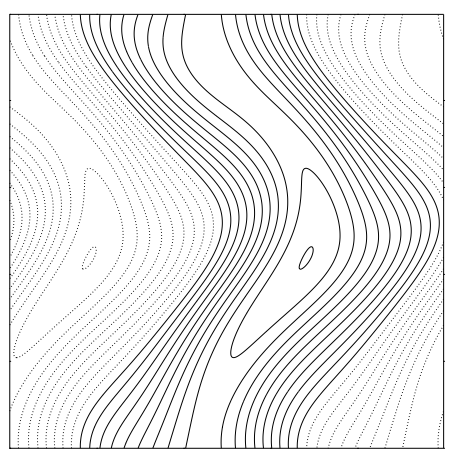

(a)

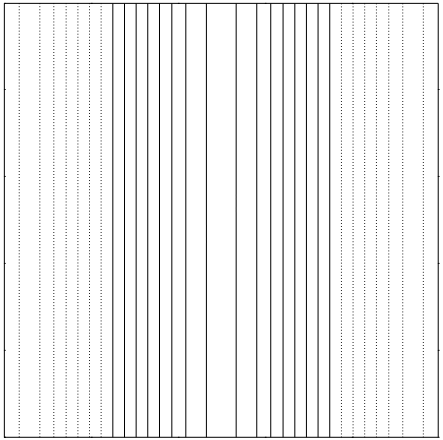

(b)

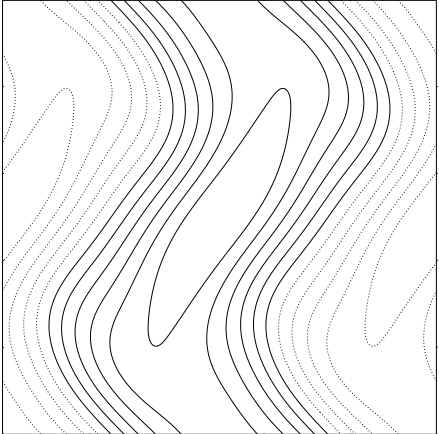

(c)

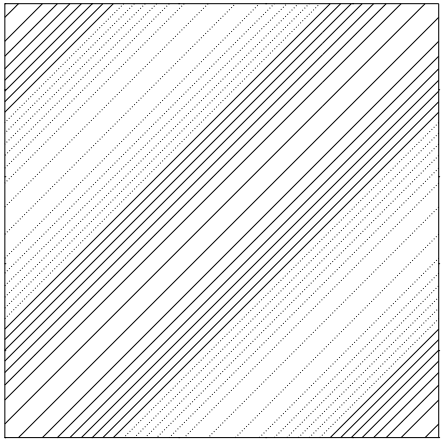

(d)

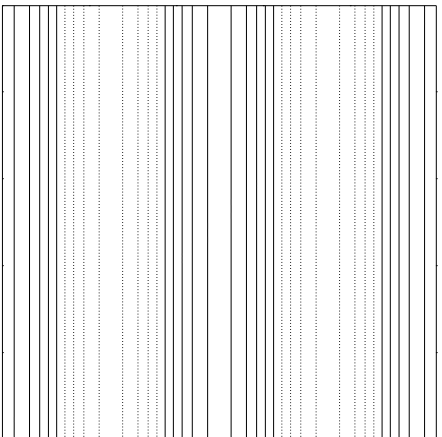

(e)

Figure 2: Isolines (step 2) of $v_{z}$ on the horizontal midplane $z=1 / 2$ for hydrodynamic attractors TW, Ta=50 (a); $\mathrm{R}_{1}$, $T a=500$ (b); WR, $T a=720$ (c); $\mathrm{R}_{\mathrm{D}}, T a=685$ (d); $\mathrm{R}_{2}, T a=1100$ (e). Solid lines indicate non-negative, dashed lines negative values. $x$ : horizontal axis, $y$ : vertical axis.

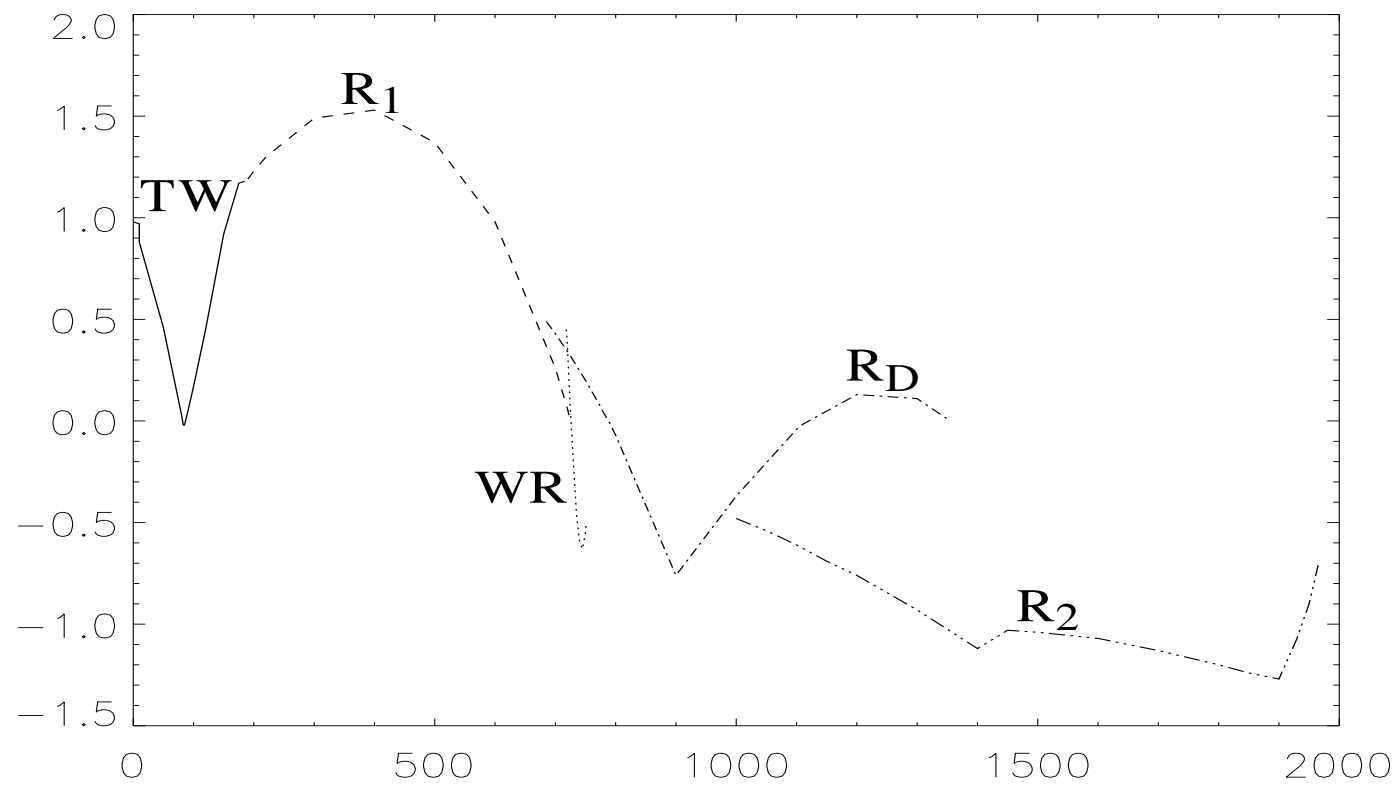

Figure 3: Growth rates (vertical axis) of magnetic field generated kinematically by hydrodynamic attractors as a function of Ta (horizontal axis). 
Table 2: Dominant kinematic magnetic modes of the hydrodynamic convective attractors. Column 2 presents generators for symmetry groups of the hydrodynamic attractors, column 3 the interval of $T a$ where a magnetic mode is dominant, column 4 dimension of the eigenspace associated with the dominant eigenvalue, column 5 the action of the symmetry group on the eigenspace, column 6 the symmetries which act trivially, the last column the eigenvalue $\lambda$ of the magnetic induction operator for which the maximal growth rate is attained in the interval of Ta specified in column 3 .

\begin{tabular}{|c|c|c|c|c|c|c|}
\hline Flow & Generators & $T a$ & $\mathrm{D}$ & Action & Kernel & $\lambda$ \\
\hline TW & $r \gamma_{L / 2}^{x}$ & {$[1,84]$} & 2 & $\mathbf{Z}_{2}$ & $q r \gamma_{L / 2}^{x}$ & $0.97 \pm 28 \mathrm{i}$ \\
& & {$[85,187]$} & 2 & $\mathbf{Z}_{2}$ & $r \gamma_{L / 2}^{x}$ & $1.18 \pm 19 \mathrm{i}$ \\
\hline $\mathrm{R}_{1}$ & $s_{2}, r \gamma_{L / 2}^{x} ; \gamma^{y}$ & {$[188,725]$} & 2 & $\mathbf{O}(2)$ & $r \gamma_{L / 2}^{x}, q \gamma_{L / 2}^{y}$ & 1.53 \\
\hline $\mathrm{WR}$ & $s_{2}, r \gamma_{L / 2}^{x}$ & {$[719,750]$} & 1 & $\mathbf{Z}_{2}$ & $q s_{2}, r \gamma_{L / 2}^{x}$ & 0.45 \\
\hline $\mathrm{R}_{\mathrm{D}}$ & $s_{2}, r \gamma_{L / 2}^{x} ; \gamma^{x y}$ & {$[684,925]$} & 2 & $\mathbf{O}(2)$ & $r q \gamma_{L / 2}^{x}, q \gamma_{L / 4}^{x y}$ & 0.49 \\
& & {$[930,1373]$} & 2 & $\mathbf{O}(2)$ & $r q \gamma_{L / 2}^{x}, q \gamma_{L / 2}^{x y}$ & 0.13 \\
\hline $\mathrm{R}_{2}$ & $s_{2}, r \gamma_{L / 4}^{x} ; \gamma^{y}$ & {$[999,1420]$} & 2 & $\mathbf{O}(2)$ & $r \gamma_{L / 4}^{x}, q \gamma_{L / 2}^{y}$ & -0.48 \\
& & {$[1430,1969]$} & 2 & $\mathbf{O}(2)$ & $r q \gamma_{L / 4}^{x y}, q \gamma_{L / 2}^{y}$ & -0.62 \\
\hline
\end{tabular}

turns out that all the attractors, except for $\mathrm{R}_{2}$, can generate magnetic field. Note, that since TW bifurcates from $\mathrm{R}_{1}$, the steady magnetic mode of $\mathrm{R}_{1}$ becomes a time-periodic mode of $\mathrm{TW}$, whose frequency at the point of bifurcation coincides with the one of TW and the eigenvalue becomes the real part of the TW magnetic eigenvalue.

There is no obvious relation between the structure of magnetic field generated by TW and stagnation (in the co-moving reference frame) points of the flow (Podvigina, 2006, found that the same was true for convective dynamos without rotation). The spatial structure of growing magnetic modes is shown on Fig. 4 and 5. For all convective flows capable of magnetic field generation, in dominant magnetic modes the field concentrates near horizontal boundaries in flattened half-ropes. A plausible underlying physical mechanism for this kind of behaviour in the case of perfectly conducting boundaries was proposed by St Pierre (1993). For steady flows, in agreement with the kinematic dynamo theory (Galloway and Zheligovsky, 1994), each half-rope is centered at a stagnation point of the flow and oriented along the one-dimensional unstable manifold of the flow at this point (see Fig. 6 (a), (c) and (e)). Magnetic field is advected by the flow and, accordingly, the ropes are stretched along the trajectories of fluid particles on the upper boundary; however, advection is affected by magnetic diffusion, which must be responsible for a (rather modest) deviation of the direction of half-ropes near their ends from the direction of the trajectories (see Fig. 6 (a), (c)). The half-ropes extend till they begin to feel the influence of the adjacent stagnation points in the direction of their stretching, which results in termination of the ropes. Magnetic field is advected by the flow into the layer; it is redistributed to form vertical two-dimensional magnetic flux sheets (shown on Fig. 6 (d) and (f) ) in the plane of the unstable/neutral directions of the stagnation points.

Sample trajectories of fluid particles inside the rolls, also shown on Fig. 6 (a), (c), (e), attest that the motion is not planar (and hence the Zeldovich, 1956, antidynamo theorem is unapplicable). In $R_{1}$ and $\mathrm{R}_{2}$ the fluid moves along closed loops, because the flows are two-dimensional (i.e. independent of a horizontal Cartesian coordinate, which in our notation is $y$ ) and possess two symmetries, $s_{2}$ (more precisely, the symmetry about any vertical line on the boundary between two adjacent rolls, rotating in opposite directions) and $r \gamma_{L / 2}^{x}$. (Due to solenoidality and two-dimensionality, $v_{x}$ and $v_{z}$ are associated with a stream function, say, $\psi(x, z)$, and equations of motion for the particles imply that their trajectories are spirals residing on the surfaces $\psi(x, z)=$ const, topologically equivalent to infinite cylinders. The two symmetries of the flow imply $\psi(x, z)=\psi(-x,-z)$ and $v_{y}(x, z)=-v_{y}(-x,-z)$, in a coordinate system with the origin on the axis of the roll; hence the helical trajectories degenerate into closed loops.) The structure of trajectories in WR is by far more complex (two trajectories in the interior of the volume of fluid are shown on Fig. 6 (c) ), however, visibly this does not affect the complexity of the magnetic field - neither at large magnetic energy levels, nor at small ones. 


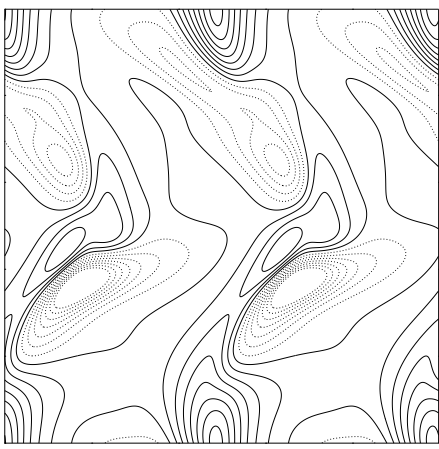

(a)

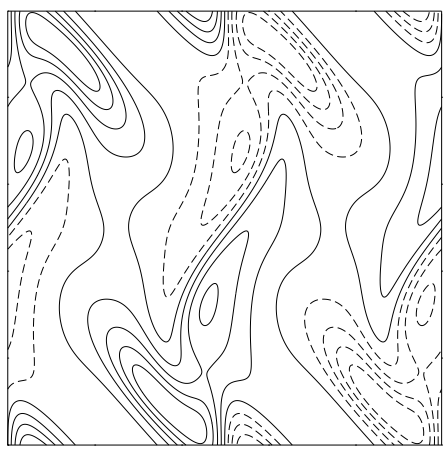

(d)

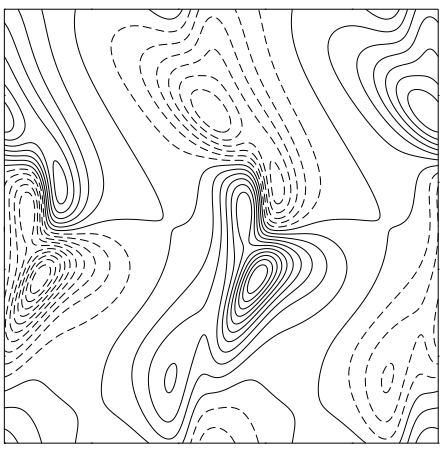

(b)

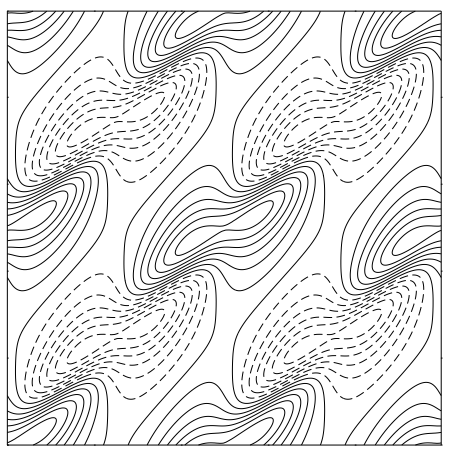

(e)

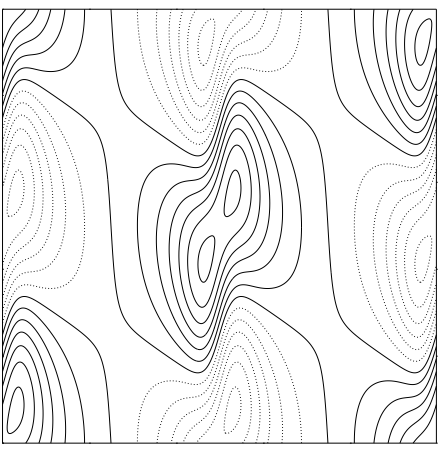

(c)

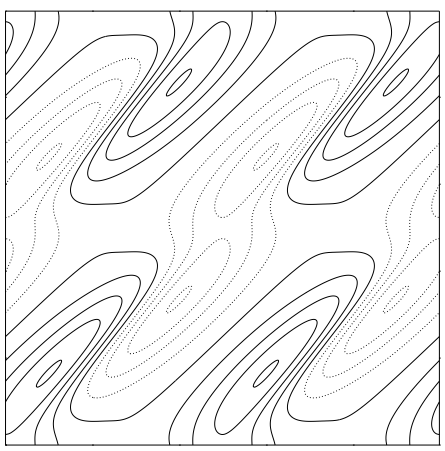

(f)

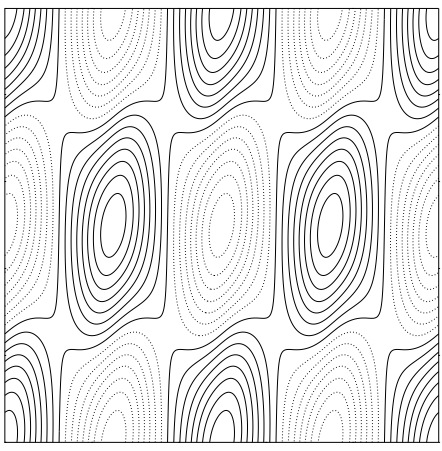

(g)

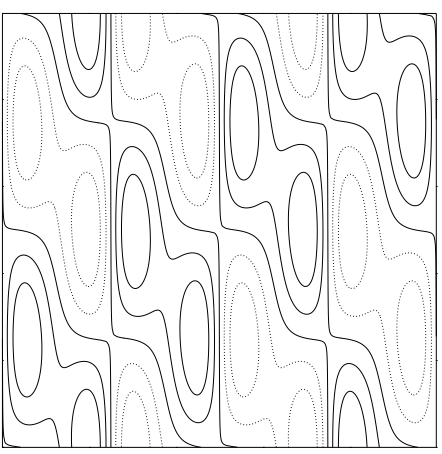

(h)

Figure 4: Isolines step 0.2 of $b_{z}$ on the horizontal midplane $z=1 / 2$ for dominant magnetic modes: $T a=50$, TW (a); Ta=150, TW (b); $T a=500, \mathrm{R}_{1}(\mathrm{c}) ; T a=720, \mathrm{WR}(\mathrm{d}) ; T a=685, \mathrm{R}_{\mathrm{D}}(\mathrm{e}) ; T a=1200, \mathrm{R}_{\mathrm{D}}(\mathrm{f}) ; T a=1100, \mathrm{R}_{2}(\mathrm{~g}) ; T a=1700, \mathrm{R}_{2}(\mathrm{~h})$. Solid lines indicate non-negative, dashed lines negative values. $x$ : horizontal axis, $y$ : vertical axis. 


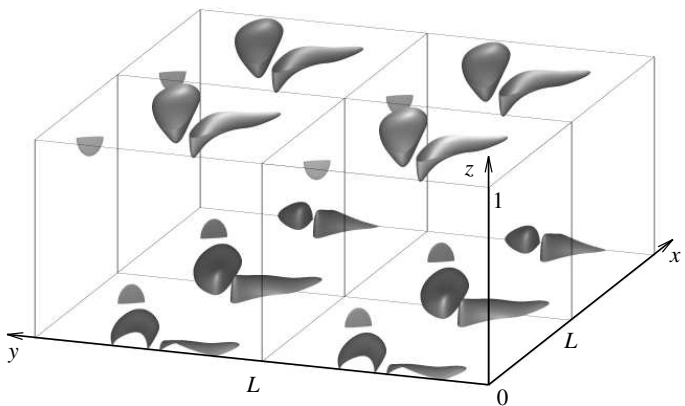

(a)

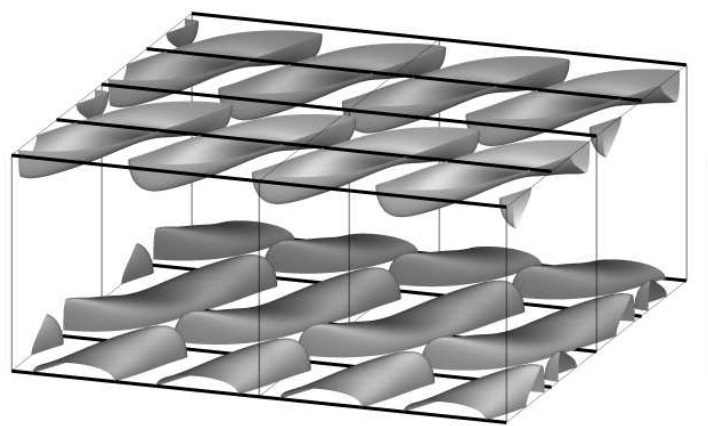

(c)

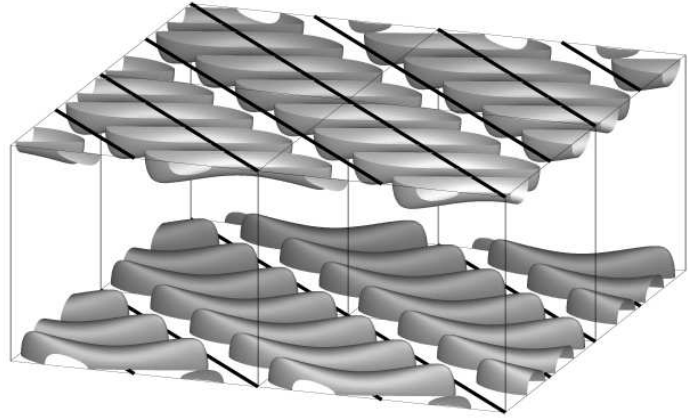

(e)

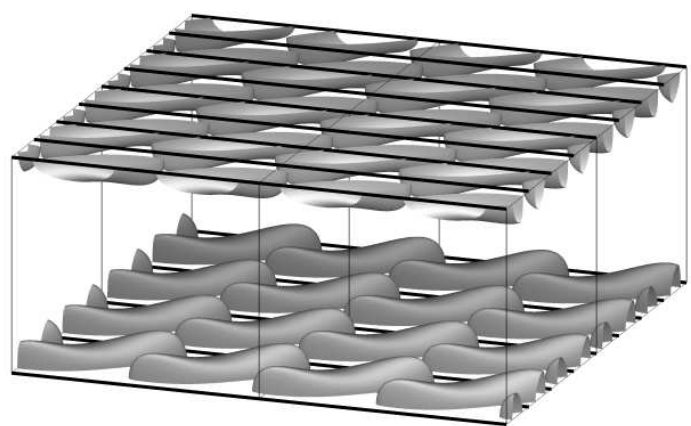

(g)

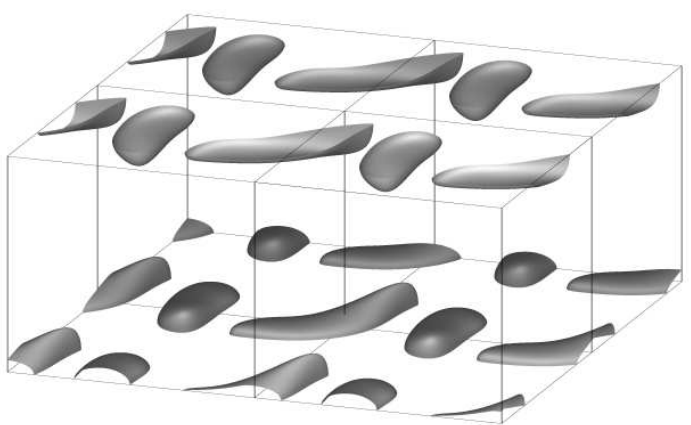

(b)

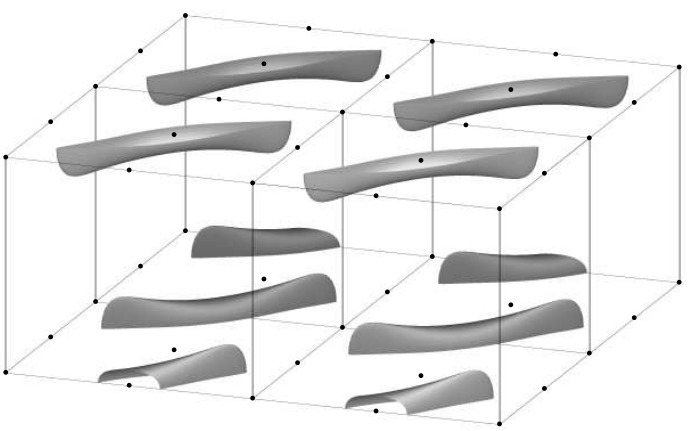

(d)

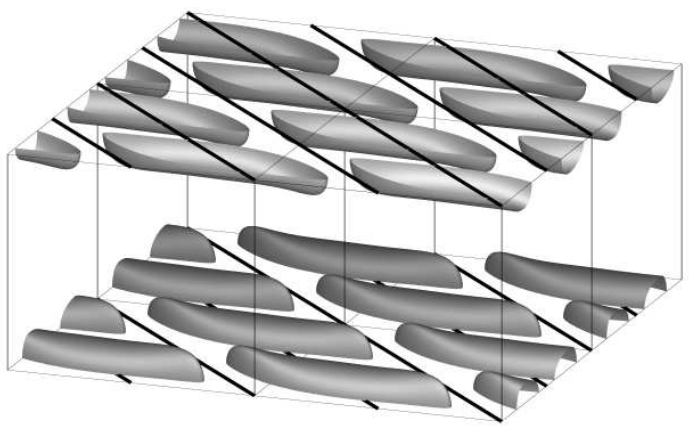

(f)

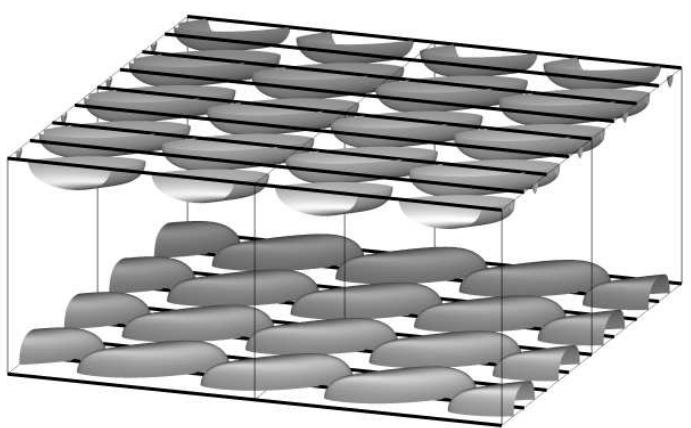

(h)

Figure 5: Isosurfaces of magnetic energy density of the dominant magnetic modes, at the level of a half of the maximum, for $T a=50, \mathrm{TW}(\mathrm{a}) ; T a=150$, TW (b); $T a=500, \mathrm{R}_{1}$ (c); $T a=720$, WR (d); $T a=685, \mathrm{R}_{\mathrm{D}}$ (e); $T a=1200, \mathrm{R}_{\mathrm{D}}(\mathrm{f}) ; T a=1100$, $\mathrm{R}_{2}(\mathrm{~g}) ; T a=1700, \mathrm{R}_{2}(\mathrm{~h})$. Isolated stagnation points and lines of stagnation points of the flow on the horizontal boundaries are shown by dots and bold lines. Four periodicity cells are displayed. On each panel, coordinate axes are as shown on (a). 


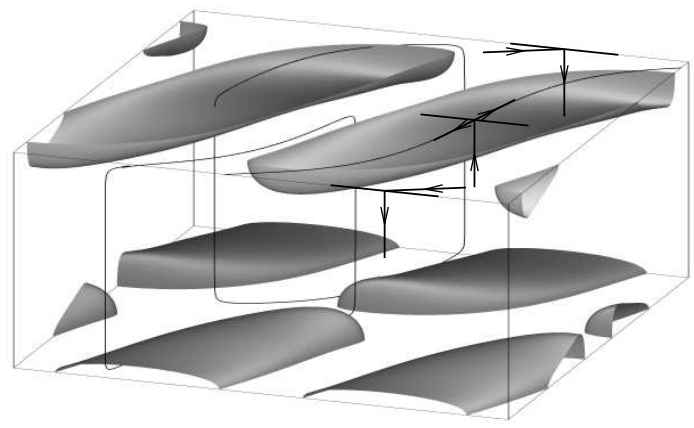

(a)

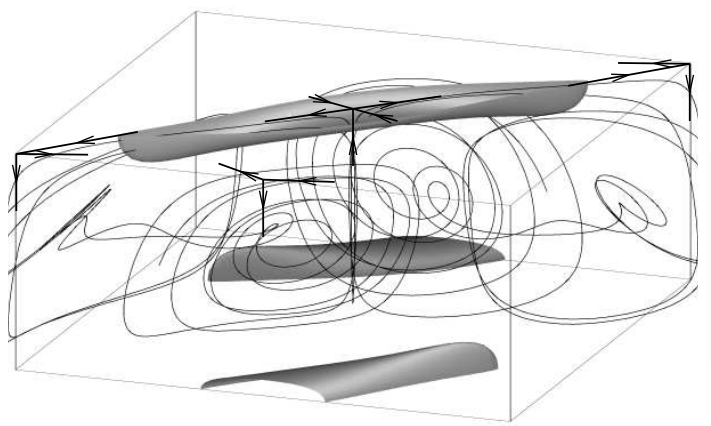

(c)

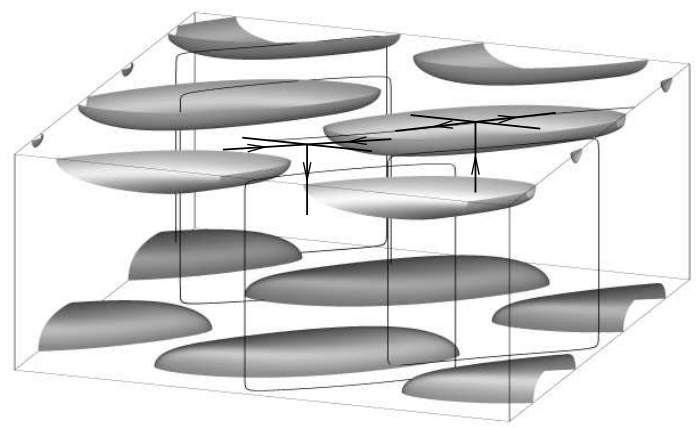

(e)

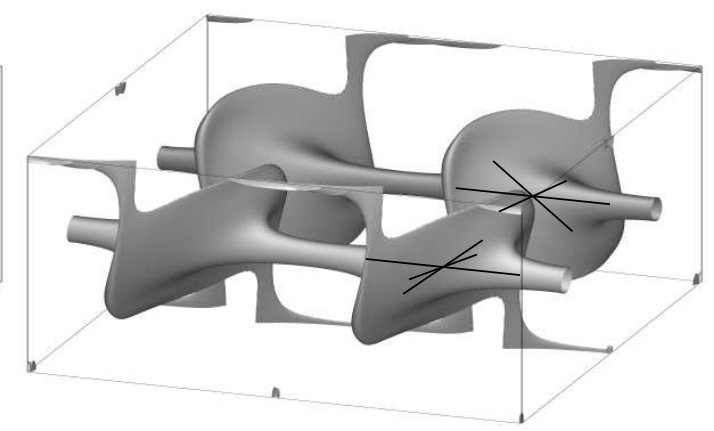

(b)

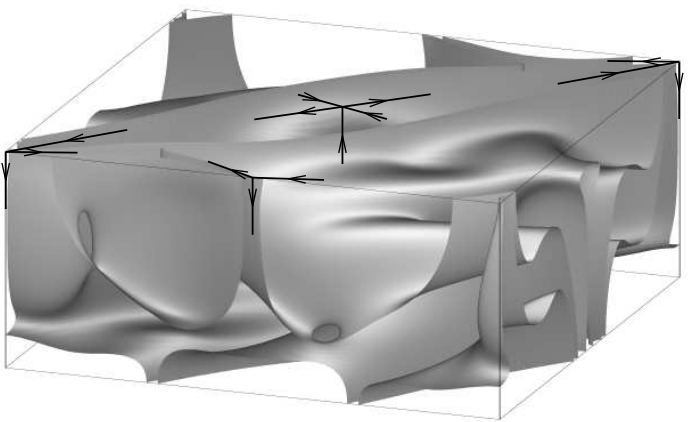

(d)

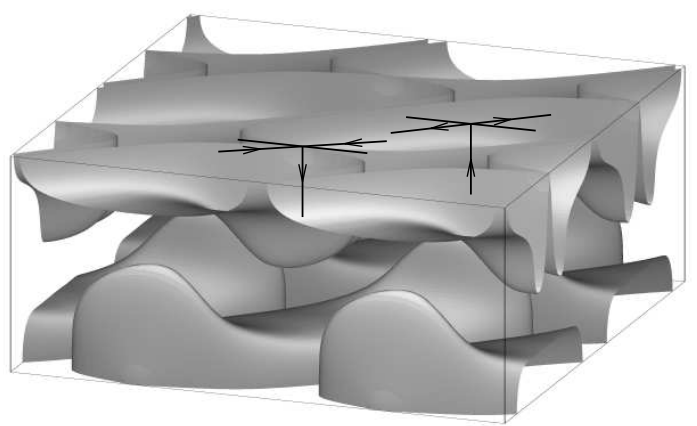

(f)

Figure 6: Isosurfaces of magnetic energy density of the dominant kinematic magnetic modes generated by $\mathrm{R}_{1}$ for $T a=500$ at the $50 \%$ level of the maximum (a) and at $3.5 \%$ (b); by WR for $T a=720$ at $50 \%$ (c) and $10 \%$ (d); by $\mathrm{R}_{2}$ at $T a=1700$ at $50 \%$ (e) and $12 \%$ (f). Stable and unstable directions of some stagnation points are shown by lines with arrows, neutral directions associated with zero and imaginary eigenvalues by lines without arrows. (See location of isolated stagnation points of the flow on the horizontal boundaries and lines of stagnation points on Fig. 5 and note that axes of rolls $\mathrm{R}_{1}$ and $\mathrm{R}_{2}$ are also comprised of stagnation points.) Sample trajectories of fluid particles on the upper boundary and in the interior of the fluid layer are shown by thin lines. One periodicity cell is displayed. On each panel, coordinate axes are as shown on Fig. 5 (a). 
Fig. 6 (b), (d), (f), showing isosurfaces of magnetic energy density at low levels, expose two-dimensional structures of magnetic field apparently related to stagnation points (for which eigenvectors of the stress tensor $\left\|\partial v_{x, y, z} / \partial\{x, y, z\}\right\|$ are also shown) of the flows. Formation of a magnetic flux sheet tangent to the two-dimensional unstable manifold of a stagnation point of a flow was investigated analytically by Childress (1979) and Childress and Soward (1985). The structures that we observe are peculiar in that magnetic energy density increases not in between the isosurfaces, as it does in magnetic flux sheets, but, on the contrary, outside them, i.e. the isosurfaces reveal two-dimensional "magnetic flux sheet gaps".

At first glance, emergence of such "antistructures" can be linked to the fact that in most cases the respective stagnation points have neutral eigendirections along lines of stagnation points (note that lines of

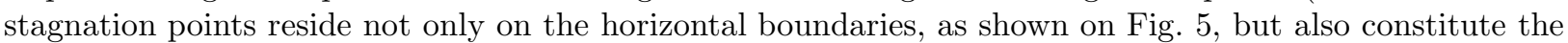
axes of the rolls $R_{1}$ and $R_{2}$ ) - at such stagnation points the two-dimensional antistructures are associated with the unstable and neutral eigendirection. In the presence of a neutral direction the theory of Childress (1979) and Childress and Soward (1985) may be unapplicable. This argument, however, breaks for isolated stagnation points of WR in the middle of upper edges parallel to the $y$ axis (see Fig. 6 (d)), which do not possess neutral eigendirections, but have genuine two-dimensional unstable manifolds. By contrast, formation of "bells of trombones", the antistructures on Fig. 6 (b), is not related to any unstable direction - they are oriented along the eigenplane associated with two imaginary eigenvalues of the stress tensor. Dynamics of fluid in this case is significantly different from that considered ibid.: the trajectories are closed oval loops centered at the axis of the roll. Fig. 目 (c) reveals, that the "bells of trombones" constitute boundaries between blobs of magnetic field of the opposite orientation.

\section{Nonlinear magnetic field generation}

Kinematic magnetic growth rates are not large (see Fig. 31). In agreement with this, for all convective MHD attractors that we have computed, magnetic energy, $E_{m}$, is smaller (at least seven times) than the kinetic one, $E_{k}$ (see Figs. 7 9). Consequently, all the MHD attractors can be regarded as perturbations of hydrodynamic attractors (note that the plot of the ratio $E_{m} / E_{k}$ is quite similar in shape to the plot of magnetic energy, cf. Figs. 9 and 8 also suggesting that we are close to the onset of both convective motions and of magnetic field generation) and the spatial structure of the flows is similar to that in the absence of magnetic field (cf. Figs. 2 and 10). Hence, we label branches of MHD attractors as follows: The main label denotes the type of an attractor: $\mathrm{S}$ for a steady state, $\mathrm{P}$ for periodic, $\mathrm{Q}$ for quasiperiodic (in assigning these labels we disregard temporal periodicities due to drifts along the $x$ and $y$ axes). The superscript denotes the hydrodynamic attractor, the MHD attractor is genetically related to. The subscript is the consecutive number within the collection of attractors of the specified morphology (e.g., there are eight different MHD steady states with spatial structure similar to that of $R_{1}$ ). The numbering of branches is in the order of increasing $T a$.

Despite hydrodynamic attractors of just four types generate magnetic field in the kinematic regime and magnetic field growth rates are not large, we have observed a large variety of MHD attractors (see Table 3 and bifurcation diagrams Figs. 7 and (8). In the description of branches of convective MHD attractors and bifurcations bounding them we follow the ordering of hydrodynamic attractors in Table 1, and for each of them we start with unstable magnetic modes. When on increasing $P_{m}$ a magnetic mode eigenvalue crosses the imaginary axis, an MHD steady state or periodic orbit appears; for supercritical bifurcations these objects are stable. We refer to them as primary magnetic attractors. For $P_{m}=8$, for which the problem is solved here, these objects are not necessarily stable; for a varying $T a$ they constitute a branch, and somewhere along the branch can gain stability. Because of emergence of the branches of unstable convective MHD states, in the present problem identification of hydrodynamic attractors with neutral kinematic magnetic modes, giving rise to the branches of primary convective MHD attractors, is not straightforward. We perform this identification by comparing the spatial structure of the fields in the attractor with the respective hydrodynamic attractor and the magnetic mode, including their symmetry groups.

To identify bifurcations of steady states we calculate eigenvalues and the associated eigenspaces of the operator of linearisation of the system (1)-(3). Hence for each bifurcation of a steady state we know the dimension and the action of the steady state symmetry group on the eigenspace (see Table 4), which is a 


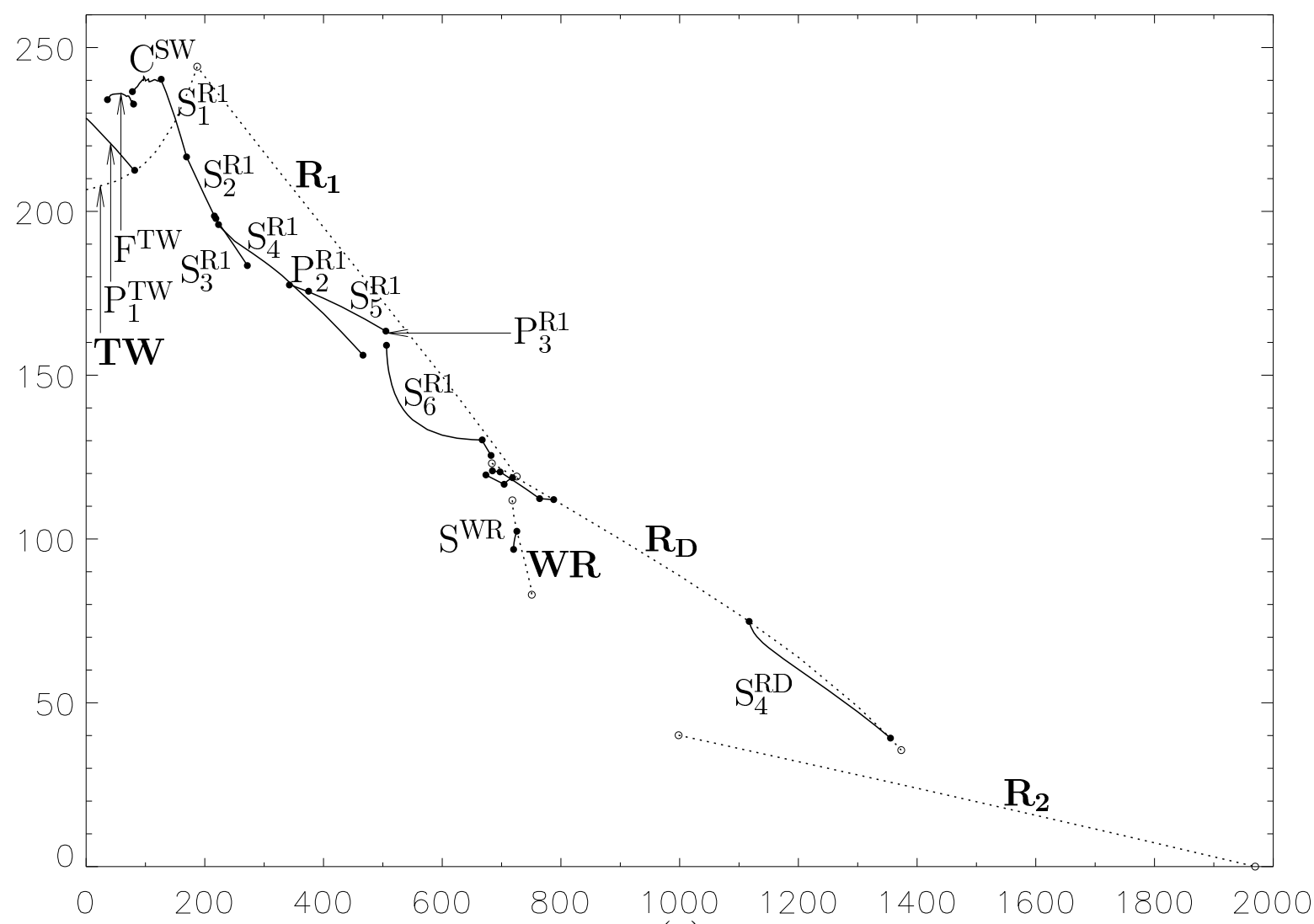

(a)

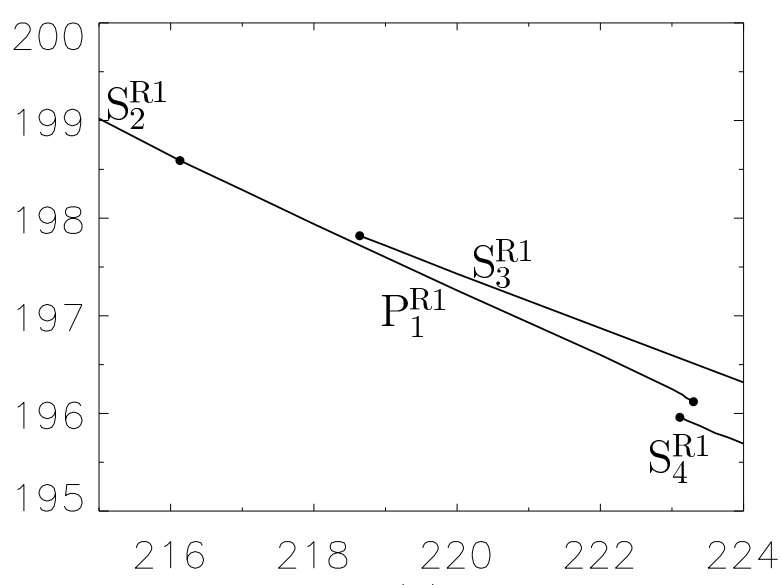

(b)

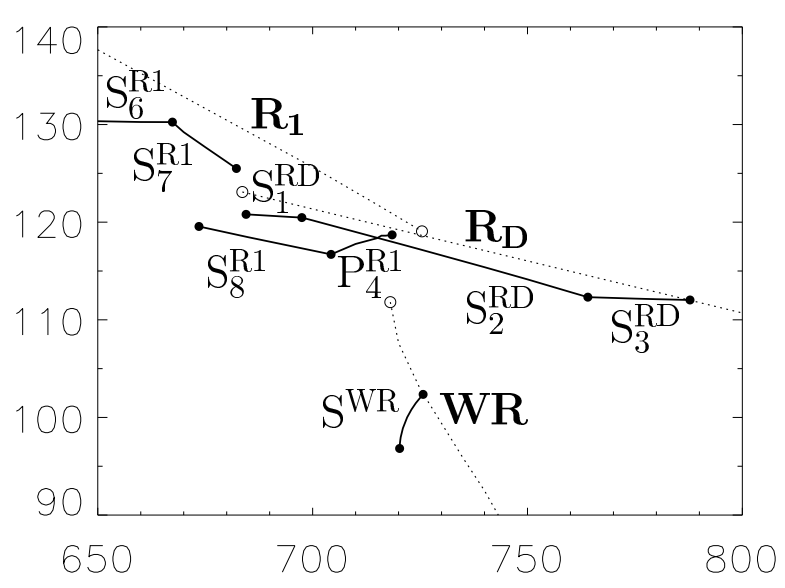

(c)

Figure 7: Kinetic $E_{k}$ energy (vertical axis) of convective MHD attractors (solid line) and of hydrodynamic attractors (dashed line) for $0<T a \leq 2000$ (horizontal axis). Labelling of attractors is explained in Sections 4 and 6 (see also Table 22). 


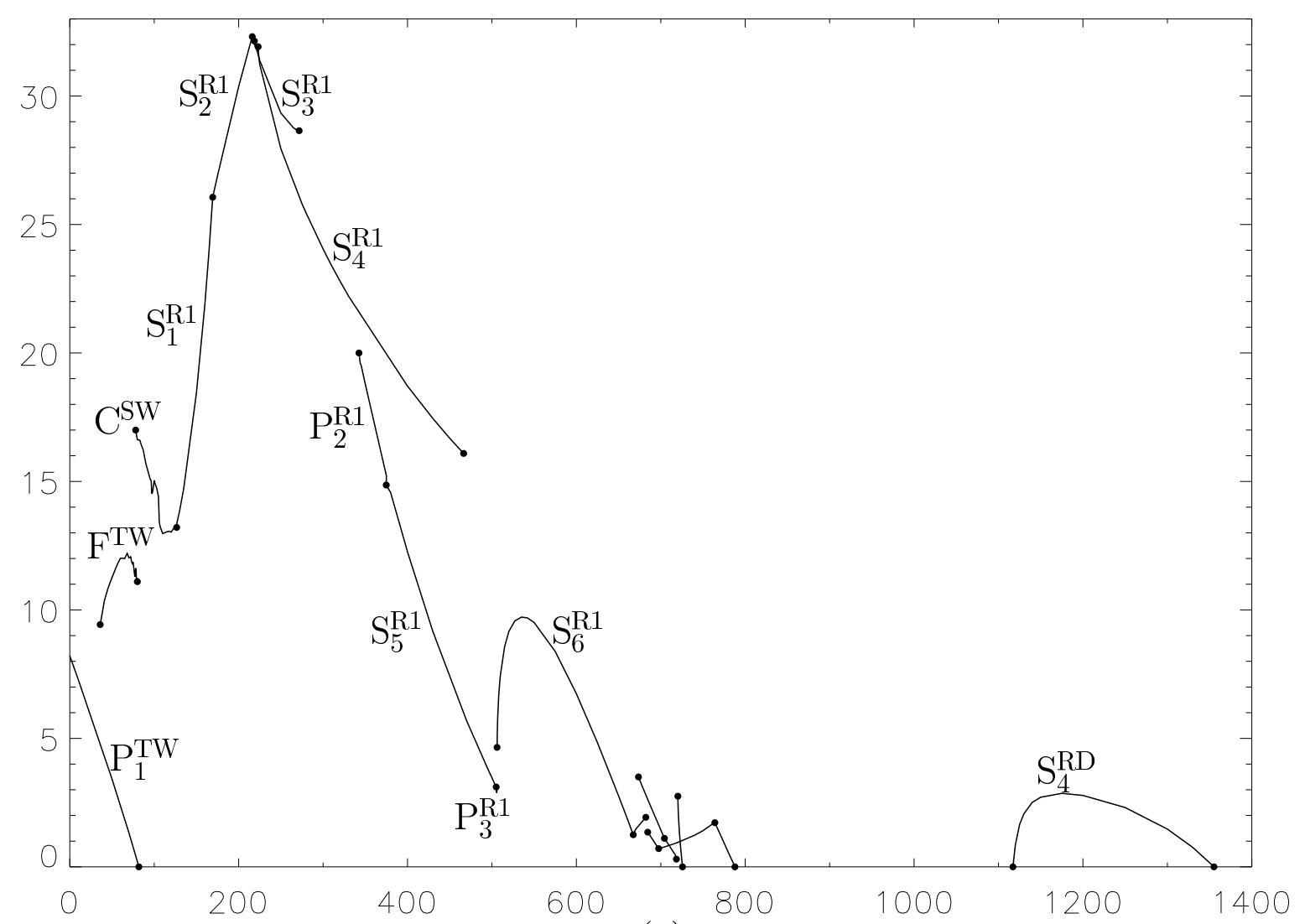

(a)

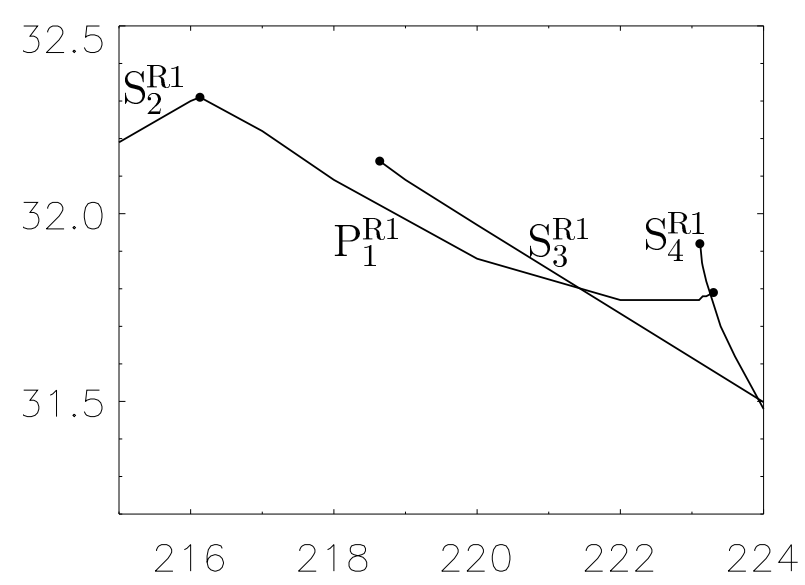

(b)

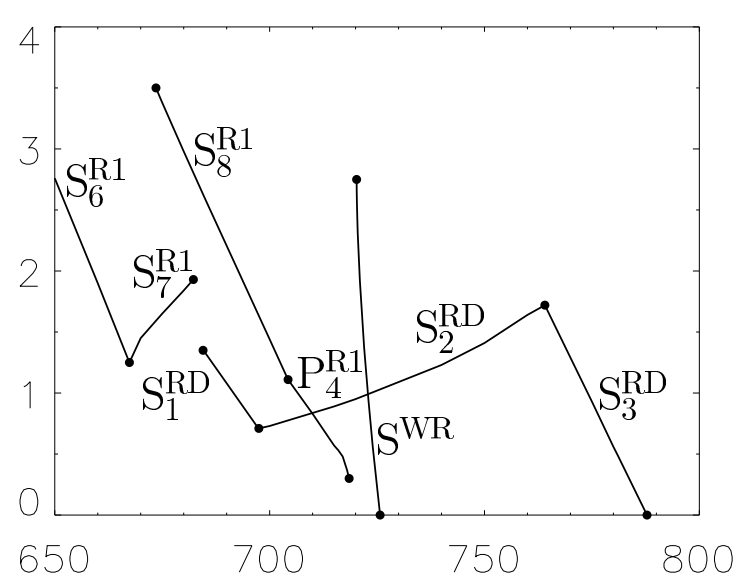

(c)

Figure 8: Magnetic $E_{m}$ energy (vertical axis) of convective MHD attractors for $0<T a \leq 2000$ (horizontal axis). Labelling of attractors is explained in Section 6 (see also Table 2). 


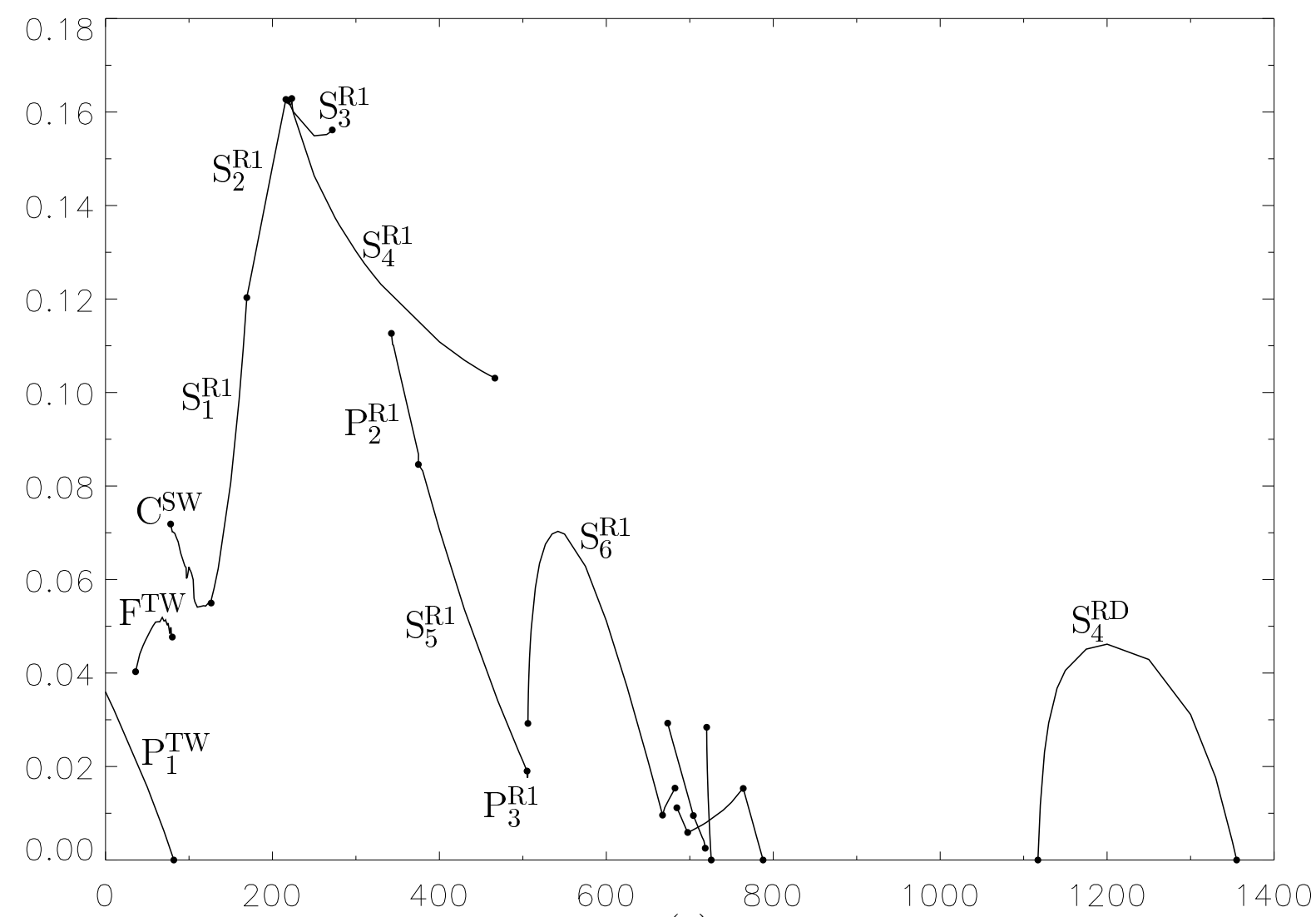

(a)

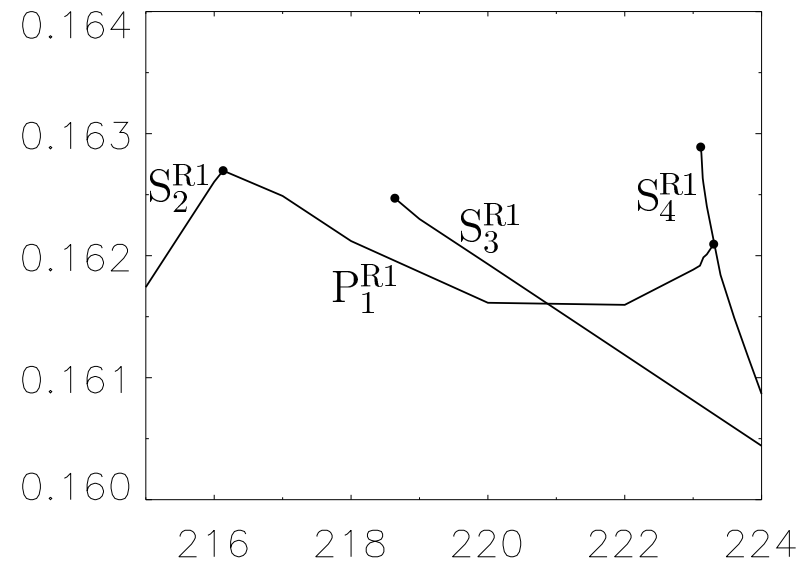

(b)

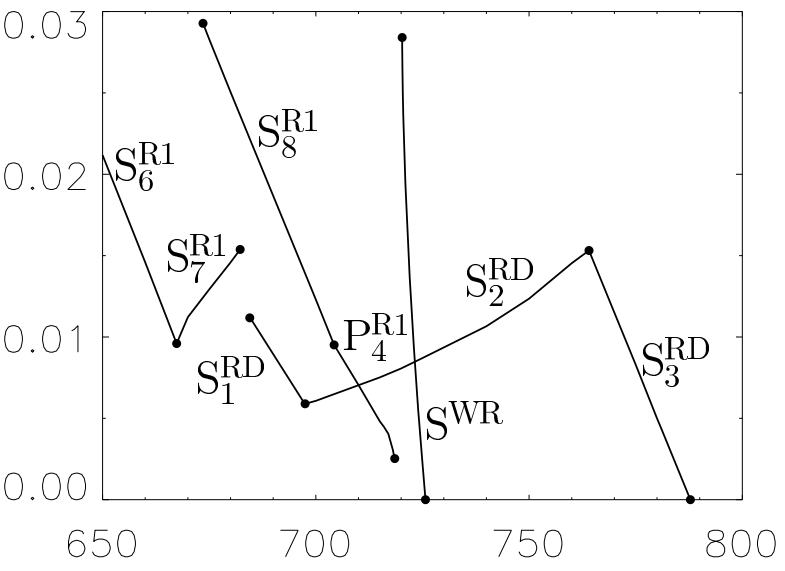

(c)

Figure 9: The ratio of energies $E_{m} / E_{k}$ (vertical axis) of MHD attractors for $0<T a \leq 2000$ (horizontal axis). 
Table 3: MHD attractors found in computations. Column 3 presents the symmetry group for which an attractor is pointwise invariant, column 4 generators of the group (for appropriately chosen location of the origin of the coordinate system); if a group is a product of several subgroups, generators of the subgroups are separated by semicolons. Columns 5 and 6 present time-averaged kinetic and magnetic energies, respectively.

\begin{tabular}{|c|c|c|c|c|c|}
\hline Type & $\begin{array}{c}\text { Interval of } \\
\text { existence }(T a) \\
\text { (frequency) }\end{array}$ & $\begin{array}{c}\text { Group of } \\
\text { symmetries }\end{array}$ & Generators & $E_{k}$ & $E_{m}$ \\
\hline $\mathrm{P}_{1}^{\mathrm{TW}}$ & $\begin{aligned} & {[1,81] } \\
f= & 4.29-4.19\end{aligned}$ & $\mathbf{Z}_{2}$ & $r q \gamma_{L / 2}^{x}$ & $228.3-212.5$ & $8.1-0$ \\
\hline $\mathrm{F}^{\mathrm{TW}}$ & {$[36,80]$} & $\mathbf{Z}_{2}$ & $r \gamma_{L / 2}^{x}$ & $234.1-232.7$ & $9.4-11.3$ \\
\hline $\mathrm{C}^{\mathrm{SW}}$ & {$[78,126]$} & $\mathbf{Z}_{2}$ & $r \gamma_{L / 2}^{x}$ & $236.6-239.9$ & $17.0-13.3$ \\
\hline $\mathrm{S}_{1}^{\mathrm{R} 1}$ & {$[127,169]$} & $\mathbf{D}_{2}$ & $r \gamma_{L / 2}^{x}, q s_{2}$ & $239.8-217.0$ & $13.4-26.0$ \\
\hline $\mathrm{S}_{2}^{\mathrm{R} 1}$ & {$[170,216]$} & $\overline{\mathbf{D}_{2} \ltimes \mathbf{Z}_{2}}$ & $r \gamma_{L / 2}^{x}, s_{2} ; q \gamma_{L / 2}^{y}$ & $216.3-198.7$ & $26.2-32.3$ \\
\hline $\mathrm{P}_{1}^{\mathrm{R} 1}$ & $\begin{array}{c}{[217,223.3]} \\
f=0.056-0.041 \\
\end{array}$ & $\mathbf{D}_{2}$ & $q \gamma_{L / 2}^{y}, r s_{2}$ & 198.3-196.1 & $32.2-31.8$ \\
\hline $\mathrm{S}_{3}^{\mathrm{RI}}$ & {$[219,271]$} & $\overline{\mathbf{D}_{2}}$ & $q \gamma_{L / 2}^{y}, s_{2}$ & $197.7-183.6$ & $32.1-28.6$ \\
\hline $\mathrm{S}_{4}^{\mathrm{R} 1}$ & {$[223.11,466]$} & $\mathbf{D}_{2}$ & $q \gamma_{L / 2}^{y}, r s_{2}$ & $196.0-156.2$ & $31.9-16.1$ \\
\hline $\mathrm{P}_{2}^{\mathrm{RI}}$ & $\begin{array}{c}{[343,377]} \\
f=0.44-0.45\end{array}$ & $\overline{\mathbf{D}_{2}}$ & $r q \gamma_{L / 2}^{x y}, q s_{2}$ & $177.6-175.4$ & $19.9-15.2$ \\
\hline $\mathrm{S}_{5}^{\mathrm{RL}}$ & {$[378,505]$} & $\overline{\mathbf{D}_{2}}$ & $r q \gamma_{L / 2}^{x y}, q s_{2}$ & $175.5-163.5$ & $15.0-3.1$ \\
\hline $\mathrm{P}_{3}^{\mathrm{RI}}$ & $\begin{array}{c}{[505.1,506.0]} \\
f=0.05-0.004\end{array}$ & $\mathbf{Z}_{2}$ & $r q \gamma_{L / 2}^{x y}$ & $163.5-163.0$ & $3.1-3.2$ \\
\hline $\mathrm{S}_{6}^{\mathrm{R} 1}$ & {$[506.1,667]$} & $\overline{\mathbf{D}_{4} \ltimes \mathbf{Z}_{2}}$ & $q \gamma_{L / 4}^{y}, s_{2} ; r q \gamma_{L / 2}^{x}$ & $159.2-130.3$ & $4.7-1.4$ \\
\hline $\mathrm{S}_{7}^{\mathrm{R} 1}$ & {$[668,682]$} & $\overline{\mathbf{D}_{2}}$ & $r q \gamma_{L / 2}^{x y}, s_{2}$ & $129.9-125.7$ & $1.3-1.9$ \\
\hline $\mathrm{S}_{8}^{\mathrm{R} 1}$ & {$[674,704]$} & $\mathbf{D}_{2}$ & $r \gamma_{L / 2}^{x}, s_{2}$ & $119.5-116.8$ & $3.4-1.1$ \\
\hline $\mathrm{P}_{4}^{\mathrm{R} 1}$ & $\begin{array}{c}{[705,718]} \\
f=0.06-0.01\end{array}$ & $\overline{\mathbf{D}_{2}}$ & $r \gamma_{L / 2}^{x}, s_{2}$ & $116.5-118.7$ & $0.9-0.4$ \\
\hline $\mathrm{S}^{\mathrm{WR}}$ & {$[721,725]$} & $\mathbf{D}_{2}$ & $r \gamma_{L / 2}^{x}, q s_{2}$ & $98.9-102.0$ & $1.9-0.2$ \\
\hline $\mathrm{S}_{1}^{\mathrm{RD}}$ & {$[685,697]$} & $\overline{\mathbf{D}_{6} \ltimes \mathbf{Z}_{2}}$ & $q \gamma_{L / 6}^{x y}, s_{2} ; r \gamma_{L / 2}^{x}$ & $120.8-120.5$ & $1.3-0.7$ \\
\hline $\mathrm{S}_{2}^{\mathrm{RD}}$ & {$[698,764]$} & $\mathbf{D}_{2}$ & $r q \gamma_{L / 2}^{y}, s_{2}$ & $120.4-112.7$ & $0.7-1.7$ \\
\hline $\mathrm{S}_{3}^{\mathrm{RD}}$ & {$[765,787]$} & $\mathbf{D}_{4} \ltimes \mathbf{Z}_{2}$ & $q \gamma_{L / 4}^{x y}, s_{2} ; r q \gamma_{L / 2}^{x}$ & $112.6-112.0$ & $1.6-0$. \\
\hline $\mathrm{S}_{4}^{\mathrm{RD}}$ & {$[1118,1355]$} & $\overline{\mathbf{D}_{2} \ltimes \mathbf{Z}_{2}}$ & $r \gamma_{L / 2}^{y}, s_{2} ; r q \gamma_{L / 2}^{x}$ & $74.3-39.2$ & $0 .-2.9$ \\
\hline
\end{tabular}


Table 4: Bifurcations of MHD steady states. Column 2 presents the symmetry group of a steady state, column 3 the generators of the symmetry group, column 4 the critical $T a$, column 5 the type of a bifurcation (S.P. denotes subcritical pitchfork, S.H. subcritical Hopf and S. saddle-node bifurcations), column 6 the dimension of the respective center eigenspace, column 7 the action of the system symmetry group on the eigenspace, and the last column elements of the group which act trivially. Note that $s_{2}$ mentioned in columns 3 and 8 have the same axis.

\begin{tabular}{|c|c|c|c|c|c|c|c|}
\hline Type & $\begin{array}{l}\text { Symmetry } \\
\text { group }\end{array}$ & Generators & $T a$ & $\overline{\mathrm{B}}$ & $\mathrm{D}$ & Action & Kernel \\
\hline $\mathrm{S}_{1}^{\mathrm{R} 1}$ & $\overline{\mathbf{D}_{2}}$ & $r \gamma_{L / 2}^{x}, q s_{2}$ & $\begin{array}{l}126.55 \\
169.27\end{array}$ & $\begin{array}{l}\text { to } \mathrm{P}^{\mathrm{SW}} \\
\text { from } \mathrm{S}_{2}^{\mathrm{R} 1}\end{array}$ & 2 & $\overline{\mathbf{Z}_{2}}$ & $r \gamma_{L / 2}^{x}$ \\
\hline $\mathrm{S}_{2}^{\mathrm{R} 1}$ & $\overline{\mathbf{D}_{2} \ltimes \mathbf{Z}_{2}}$ & $r \gamma_{L / 2}^{x}, s_{2} ; q \gamma_{L / 2}^{y}$ & $\begin{array}{l}169.27 \\
216.13\end{array}$ & $\begin{array}{l}\text { to } \mathrm{S}_{1}^{\mathrm{R} 1} \\
\text { to } \mathrm{P}_{1}^{\mathrm{R} 1}\end{array}$ & $\begin{array}{l}1 \\
2\end{array}$ & $\begin{array}{l}\mathbf{Z}_{2} \\
\mathbf{Z}_{2}\end{array}$ & $\begin{array}{l}r \gamma_{L / 2}^{x}, q s_{2} \gamma_{L / 2}^{y} \\
r s_{2} \gamma_{L / 2}^{x}, q \gamma_{L / 2}^{y}\end{array}$ \\
\hline $\mathrm{S}_{3}^{\mathrm{RI}}$ & $\overline{\mathbf{D}_{2}}$ & $q \gamma_{L / 2}^{y}, s_{2}$ & $\begin{array}{l}218.64 \\
271.66\end{array}$ & $\begin{array}{l}\text { S.H. } \\
\text { S.P. }\end{array}$ & $\begin{array}{l}2 \\
1\end{array}$ & $\begin{array}{l}\mathbf{Z}_{2} \\
\mathbf{Z}_{2}\end{array}$ & $\begin{array}{l}q \gamma_{L / 2}^{y} \\
q \gamma_{L / 2}^{y}\end{array}$ \\
\hline $\mathrm{S}_{4}^{\mathrm{RT}}$ & $\overline{\mathbf{D}_{2}}$ & $q \gamma_{L / 2}^{y}, r s_{2}$ & $\begin{array}{l}223.10 \\
466.50\end{array}$ & $\begin{array}{l}\text { S. } \\
\text { S.P. }\end{array}$ & $\begin{array}{l}1 \\
1\end{array}$ & $\begin{array}{c}\mathbf{1} \\
\mathbf{Z}_{2}\end{array}$ & $\begin{array}{c}q \gamma_{L / 2}^{y}, r s_{2} \\
q r s_{2} \gamma_{L / 2}^{y}\end{array}$ \\
\hline $\mathrm{S}_{5}^{\mathrm{R} 1}$ & $\overline{\mathbf{D}_{2}}$ & $r q \gamma_{L / 2}^{x y}, q s_{2}$ & $\begin{array}{l}377.89 \\
505.05\end{array}$ & $\begin{array}{l}\text { to } \mathrm{P}_{2}^{\mathrm{R} 1} \\
\text { to } \mathrm{P}_{3}^{\mathrm{R} 1}\end{array}$ & $\begin{array}{l}2 \\
2\end{array}$ & $\begin{array}{c}\mathbf{1} \\
\mathbf{Z}_{2}\end{array}$ & $\begin{array}{c}r q \gamma_{L / 2}^{x y}, q s_{2} \\
r q \gamma_{L / 2}^{x y}\end{array}$ \\
\hline $\mathrm{S}_{6}^{\mathrm{R} 1}$ & $\mathbf{D}_{4} \ltimes \mathbf{Z}_{2}$ & $q \gamma_{L / 4}^{y}, s_{2} ; r q \gamma_{L / 2}^{x}$ & $\begin{array}{l}506.07 \\
667.37\end{array}$ & $\begin{array}{c}\mathrm{S} . \\
\text { to } \mathrm{S}_{7}^{\mathrm{R} 1}\end{array}$ & $\begin{array}{l}1 \\
2\end{array}$ & $\begin{array}{c}1 \\
\mathbf{D}_{4}\end{array}$ & $\begin{array}{c}q \gamma_{L / 4}^{y}, s_{2} ; r q \gamma_{L / 2}^{x} \\
r q \gamma_{L / 2}^{x y}\end{array}$ \\
\hline $\mathrm{S}_{7}^{\mathrm{R} 1}$ & $\mathbf{D}_{2}$ & $r q \gamma_{L / 2}^{x y}, s_{2}$ & $\begin{array}{l}667.37 \\
682.26\end{array}$ & $\begin{array}{c}\text { from } S_{6}^{\mathrm{R} 1} \\
\text { S.P. }\end{array}$ & 1 & $\mathbf{Z}_{2}$ & $s_{2}$ \\
\hline $\mathrm{S}_{8}^{\mathrm{R} 1}$ & $\mathbf{D}_{2}$ & $r \gamma_{L / 2}^{x}, s_{2}$ & $\begin{array}{l}673.55 \\
704.29\end{array}$ & $\begin{array}{c}\text { S.P. } \\
\text { to } \mathrm{P}_{4}^{\mathrm{R} 1}\end{array}$ & $\begin{array}{l}1 \\
2\end{array}$ & $\begin{array}{c}\mathbf{Z}_{2} \\
\mathbf{1}\end{array}$ & $\begin{array}{c}s_{2} \\
r \gamma_{L / 2}^{x}, s_{2}\end{array}$ \\
\hline $\mathrm{S}^{\mathrm{WR}}$ & $\overline{\mathbf{D}_{2}}$ & $r \gamma_{L / 2}^{x}, q s_{2}$ & $\begin{array}{l}720.25 \\
725.71\end{array}$ & $\begin{array}{c}\text { S. } \\
\text { from WR }\end{array}$ & 1 & $\mathbf{1}$ & $r \gamma_{L / 2}^{x}, q s_{2}$ \\
\hline $\mathrm{S}_{1}^{\mathrm{RD}}$ & $\mathbf{D}_{6} \ltimes \mathbf{Z}_{2}$ & $q \gamma_{L / 6}^{x y}, s_{2} ; r \gamma_{L / 2}^{x}$ & $\begin{array}{l}684.51 \\
697.49 \\
\end{array}$ & $\begin{array}{c}\text { S.P. } \\
\text { to } \mathrm{S}_{2}^{\mathrm{RD}}\end{array}$ & $\begin{array}{l}2 \\
2\end{array}$ & $\begin{array}{l}\mathbf{D}_{6} \\
\mathbf{D}_{6} \\
\end{array}$ & $\begin{array}{r}r \gamma_{L / 2}^{x} \\
r q \gamma_{L / 2}^{y}\end{array}$ \\
\hline $\mathrm{S}_{2}^{\mathrm{RD}}$ & $\overline{\mathbf{D}_{2}}$ & $s_{2}, r q \gamma_{L / 2}^{y}$ & $\begin{array}{l}697.49 \\
764.05\end{array}$ & $\begin{array}{l}\text { from } S_{1}^{R D} \\
\text { from } S_{3}^{R D}\end{array}$ & & & \\
\hline $\mathrm{S}_{3}^{\mathrm{RD}}$ & $\overline{\mathbf{D}_{4} \ltimes \mathbf{Z}_{2}}$ & $q \gamma_{L / 4}^{x y}, s_{2} ; r q \gamma_{L / 2}^{x}$ & $\begin{array}{l}764.05 \\
787.82\end{array}$ & $\begin{array}{c}\text { to } \mathrm{S}_{2}^{\mathrm{RD}} \\
\text { from RD }\end{array}$ & 2 & $\overline{\mathbf{D}_{4}}$ & $r q \gamma_{L / 2}^{y}$ \\
\hline $\mathrm{S}_{4}^{\mathrm{RD}}$ & $\overline{\mathbf{D}_{2} \ltimes \mathbf{Z}_{2}}$ & $r \gamma_{L / 2}^{y}, s_{2} ; r q \gamma_{L / 2}^{x}$ & $\begin{array}{l}1117.47 \\
1355.20\end{array}$ & $\begin{array}{l}\text { from RD } \\
\text { from RD }\end{array}$ & & & \\
\hline
\end{tabular}




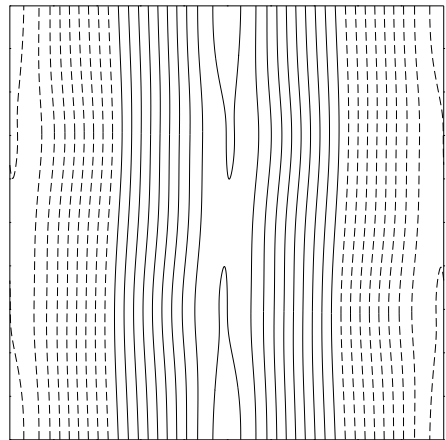

(a)

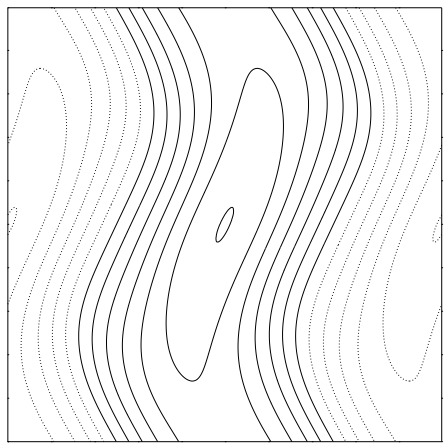

(c)

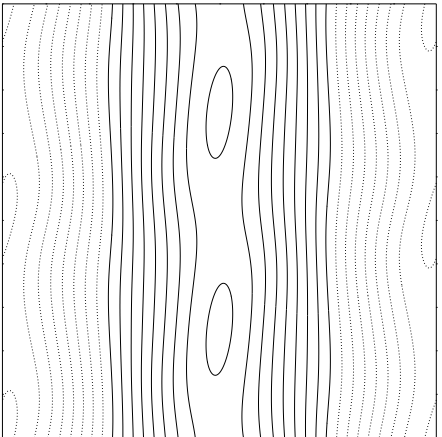

(b)

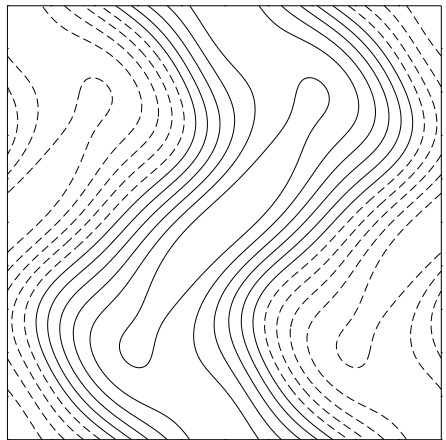

(d)

Figure 10: Isolines (step 2) of $v_{z}$ on the horizontal midplane $z=1 / 2$ for some steady convective MHD attractors: $\mathrm{S}_{1}^{\mathrm{R} 1}, T a=150$ (a); $\mathrm{S}_{3}^{\mathrm{R} 1}, T a=250$ (b); $\mathrm{S}_{8}^{\mathrm{R} 1}, T a=680$ (c); $\mathrm{S}^{\mathrm{WR}}, T a=721$ (d). Solid lines indicate non-negative, dashed lines negative values. $x$ : horizontal axis, $y$ : vertical axis.

necessary information for application of the general theory of bifurcations for symmetric systems (Golubitsky et al., 1988).

For each growing magnetic mode there exists a primary MHD attractor. At points of bifurcations from rolls, dimension of the kernel of the magnetic induction operator is two due to the presence of translation symmetries (see Table 2). The action of the symmetry group on the eigenspace is $\mathbf{O}(2)$ generated by $s_{2}$ and translations. Along axes of rolls eigenmodes can have periods $\ell / n$ with an integer $n>0$, where for rolls parallel to coordinate axes $\ell=L$ and for rolls parallel to a diagonal $\ell=\sqrt{2} L$. If a magnetic mode has a period $\ell / n$, the shift by $\ell /(2 n)$ acts as $-I$, hence the superposition of the shift by $\ell /(2 n)$ and $q$ maps the mode into itself. Bifurcations from rolls are pitchfork with the symmetry group $\mathbf{O}(2)$. Thus a continuum of steady states emerges, the symmetry group of each of them is a product of $\mathbf{Z}_{2}$ generated by $s_{2}$ and the subgroup which acts trivially. Bifurcations from TW are Hopf ones, and for WR it is pitchfork.

The spatial structure of magnetic fields is shown on Fig. 11] A common dominant feature in the majority of nonlinear convective hydromagnetic regimes is concentration of magnetic field flux in half-ropes located near the horizontal boundaries. In the primary attractors this structure is inherited from the respective kinematic dynamo modes. Concentration of magnetic field near boundaries of the layer was observed by St Pierre (1993) in simulations of subcritical magnetic field generation by thermal convection of rapidly rotating fluid (for the same boundary conditions, as employed here). Such behaviour of magnetic field was observed in many computations (see a discussion and references in Zheligovsky, 2010) and it is usually expected for electrically perfectly conducting boundaries (although Zheligovsky, 2010, found for these boundary conditions an example of a time-periodic nonlinear convective magnetic dynamo, in which magnetic field always remains concentrated inside the layer of fluid).

In what follows we overview the MHD attractors found in computations and their bifurcations. 


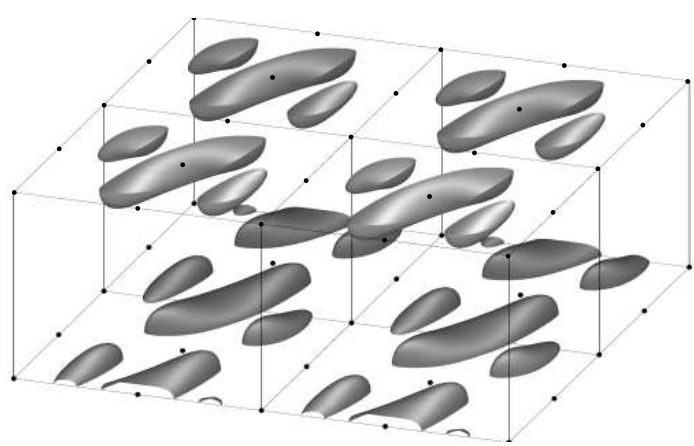

(a)

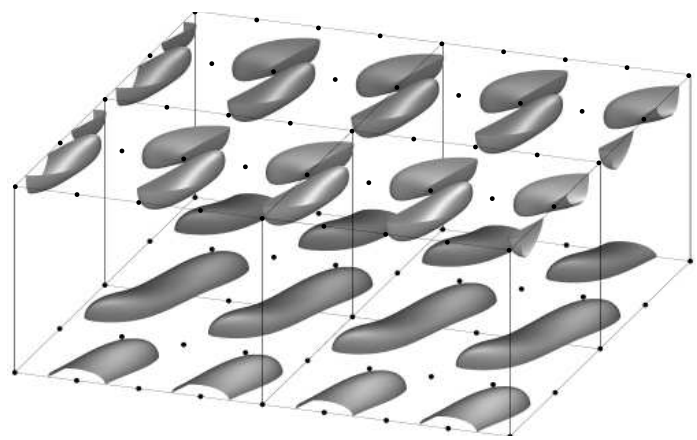

(c)

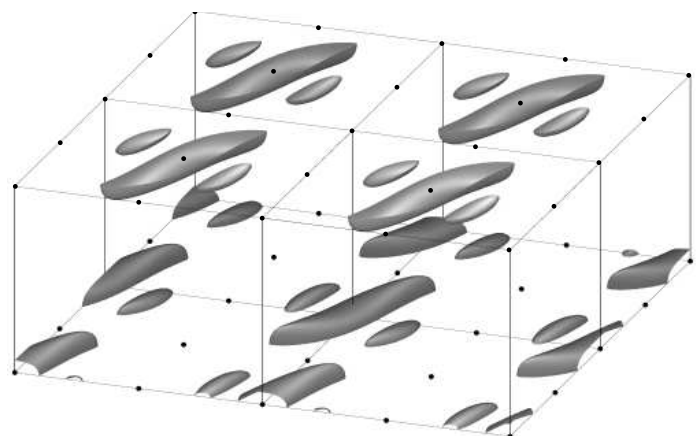

(e)

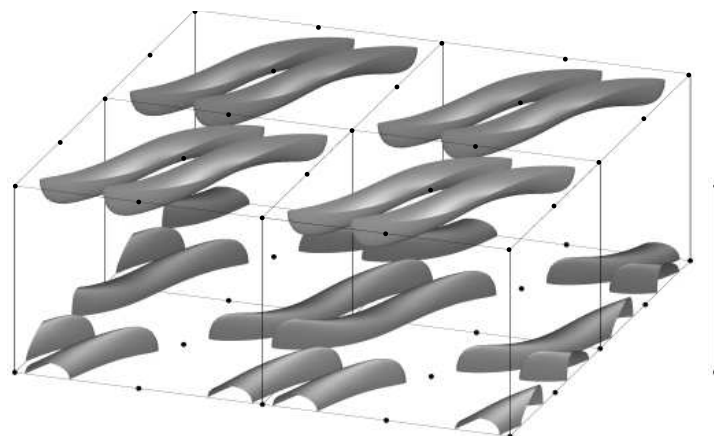

(g)

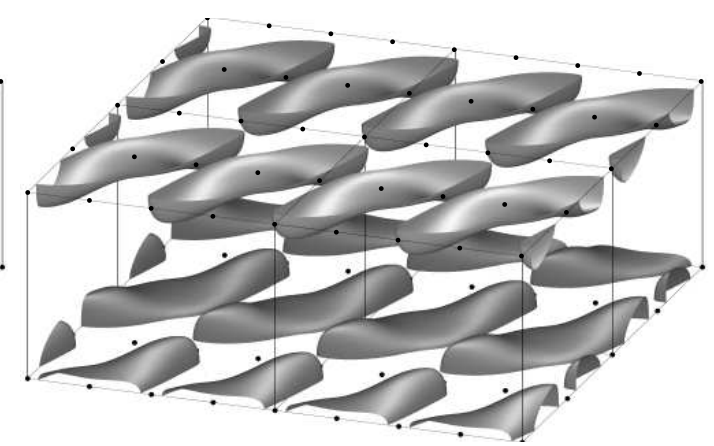

(b)

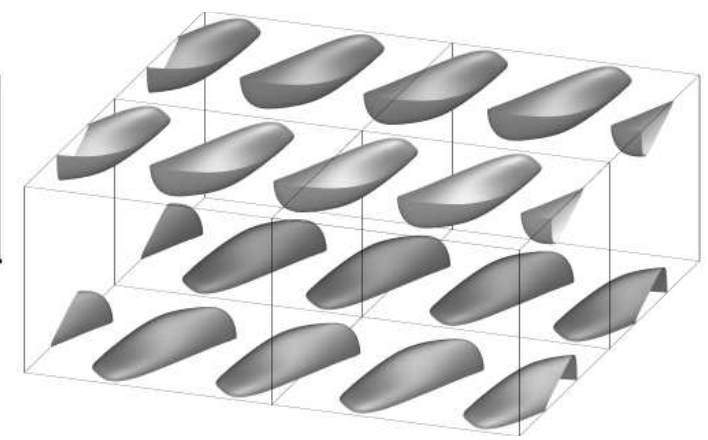

(d)

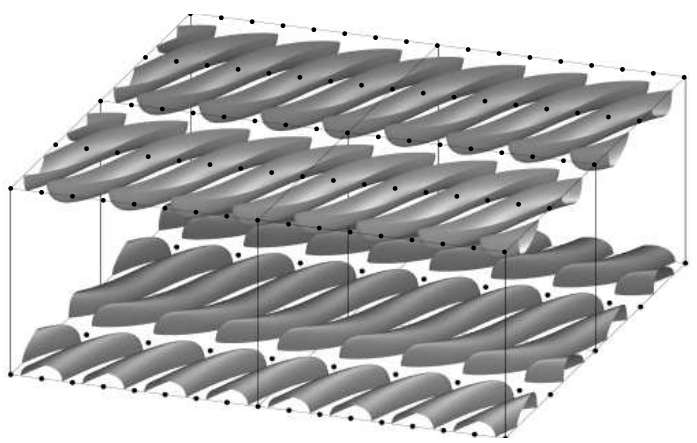

(f)

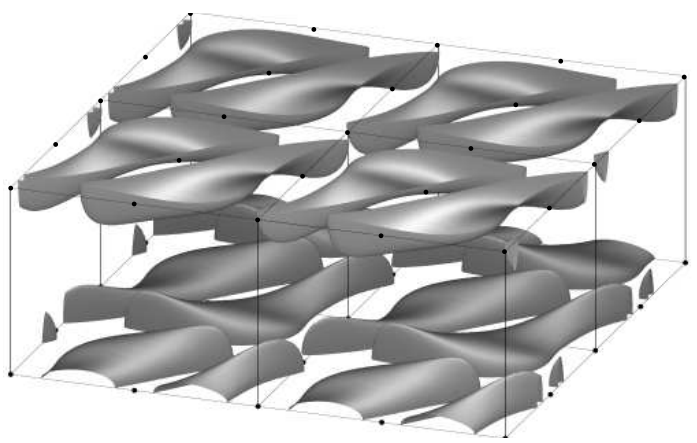

(h)

Figure 11: Isosurfaces of magnetic energy density of magnetic fields, at the level of a half of the maximum, in steady convective MHD attractors: $T a=150, \mathrm{~S}_{1}^{\mathrm{R} 1}(\mathrm{a}) ; T a=200, \mathrm{~S}_{2}^{\mathrm{R} 1}(\mathrm{~b}) ; T a=250, \mathrm{~S}_{3}^{\mathrm{R} 1}(\mathrm{c}) ; T a=300, \mathrm{~S}_{4}^{\mathrm{R} 1}(\mathrm{~d}) ; T a=430, \mathrm{~S}_{5}^{\mathrm{R} 1}(\mathrm{e}) ; T a=600$, $\mathrm{S}_{6}^{\mathrm{R} 1}(\mathrm{f}) ; T a=675, \mathrm{~S}_{7}^{\mathrm{R} 1}(\mathrm{~g}) ; T a=680, \mathrm{~S}_{8}^{\mathrm{R} 1}(\mathrm{~h}) ; T a=721, \mathrm{~S}^{\mathrm{WR}}(\mathrm{i}) ; T a=690, \mathrm{~S}_{1}^{\mathrm{RD}}(\mathrm{k}) ; T a=740, \mathrm{~S}_{2}^{\mathrm{RD}}(\mathrm{l}) ; T a=775, \mathrm{~S}_{3}^{\mathrm{RD}}$ $(\mathrm{m}) ; \mathrm{Ta}=1175, \mathrm{~S}_{4}^{\mathrm{RD}}{ }^{(\mathrm{n})}$. Stagnation points of the flow on the horizontal boundaries are shown by dots. Four periodicity cells are displayed. 


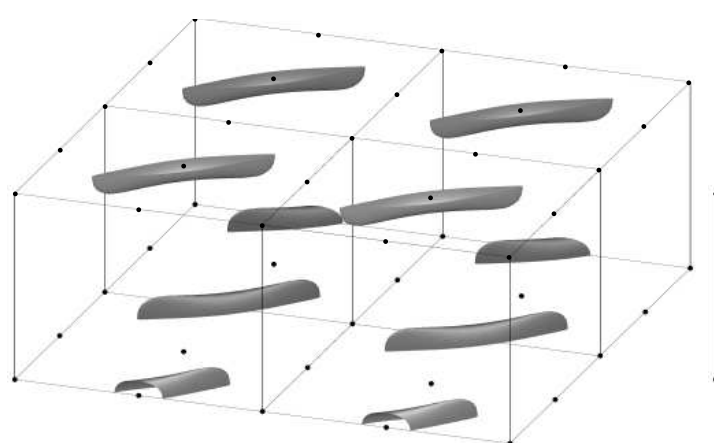

(i)

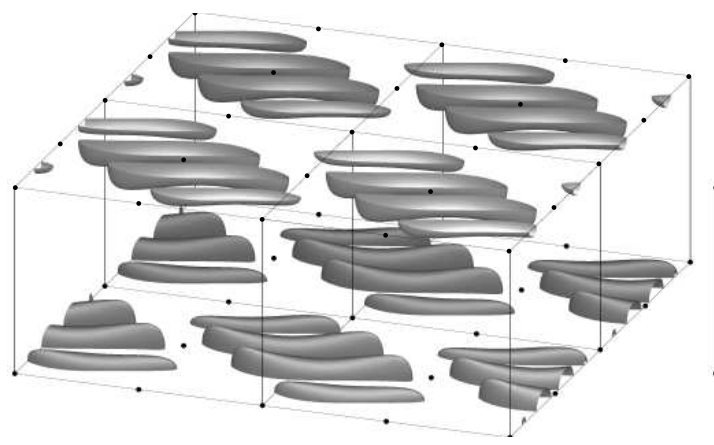

(l)

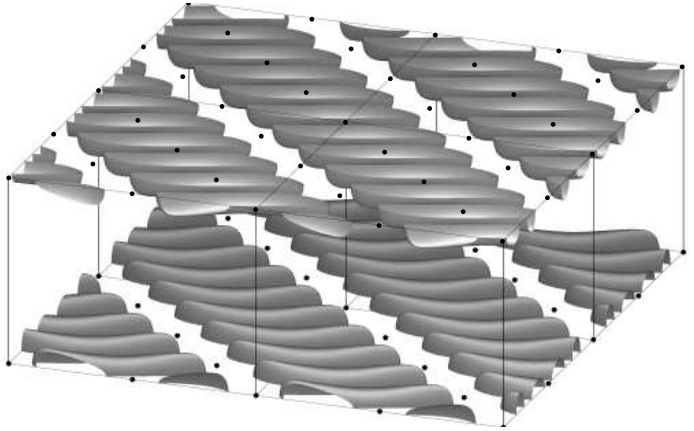

(k)

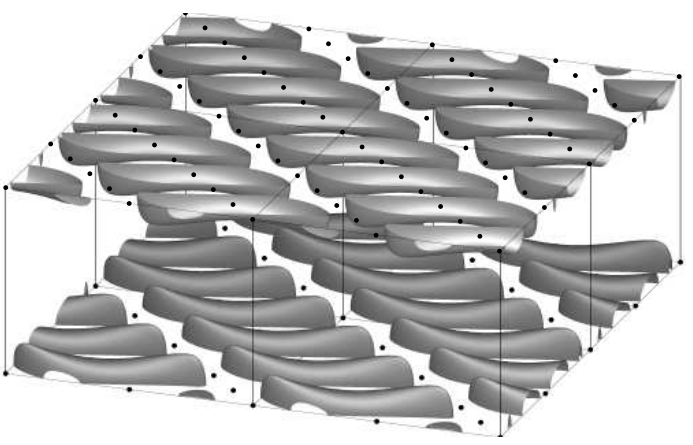

(m)

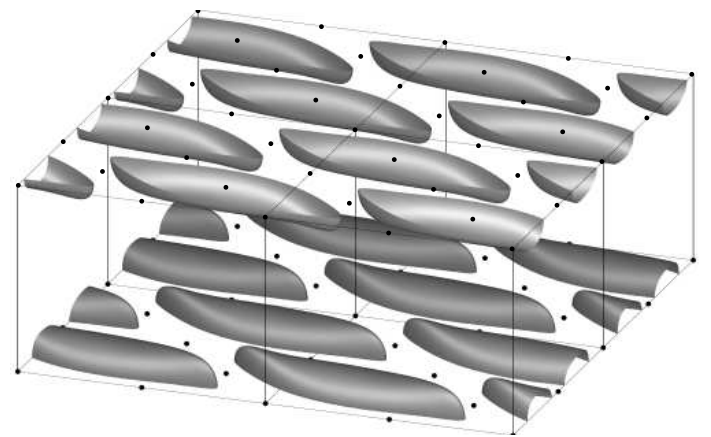

(n)

Fig. 11] continuation. 


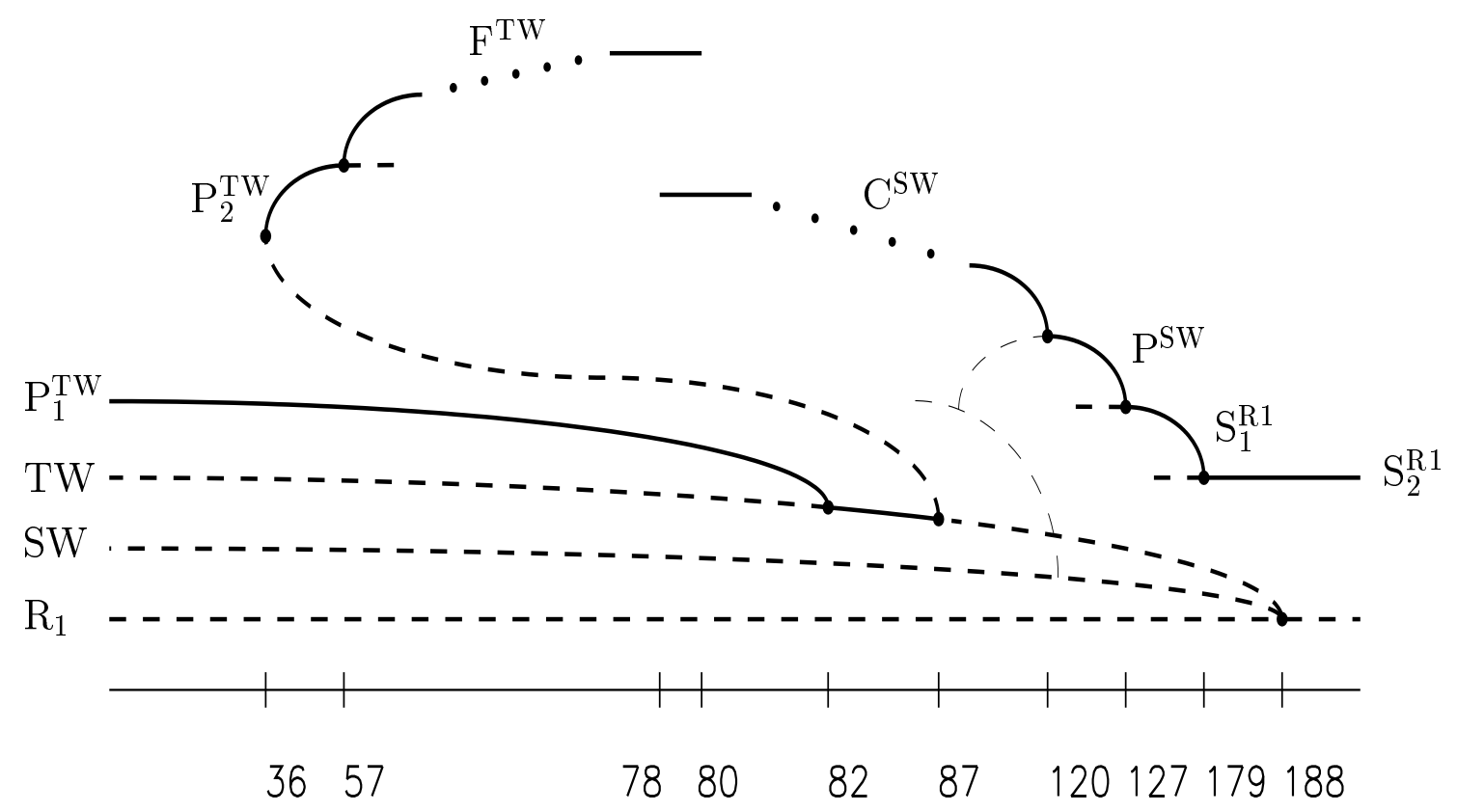

Figure 12: Bifurcation diagram of the MHD system for $0<T a<200$. Solid lines denote stable branches, dashed unstable, thin dashed conjectured.

\subsection{MHD attractors emerging from $\mathrm{TW}, 0<T a \leq 81$}

The bifurcation diagram of MHD regimes at the interval $0<T a<200$ is shown on Fig. 12 .

The interval of $T a$, where TW exists, consists of two subintervals of kinematic dynamo action, separated by a window $82 \leq T a \leq 86$ of non-generating TW regimes. Growing magnetic modes generated in the two subintervals differ, for instance, in their symmetries (see Table 21). This results in emergence of MHD attractors of different types. At the lower subinterval of Ta the primary MHD attractor is $\mathrm{P}_{1}^{\mathrm{TW}}$, similarly to TW drifting along both horizontal axes, $x$ and $y$, and periodic in the co-moving reference frame. At the right end the MHD branch $\mathrm{P}_{1}^{\mathrm{TW}}$ terminates on $\mathrm{TW}$ at $T a=81.80$, where the respective magnetic eigenvalue of TW crosses the imaginary axis; to the left it continues to $T a=0$.

\section{2. $M H D$ attractors emerging from $\mathrm{TW}$, mode interaction}

As noted in Section 3, TW bifurcates supercritically in a Hopf bifurcation from $\mathrm{R}_{1}$ as $T a$ decreases below 188. For the employed value of $P_{m}$, at the point of bifurcation $\mathrm{R}_{1}$ generates magnetic field kinematically. The respective magnetic eigenvalue is real and not large, hence attractors observed for $T a$ not far from 188 can be related to interaction of two instability modes of $\mathrm{R}_{1}$, hydrodynamic and magnetic ones.

The symmetry group of $\mathrm{R}_{1}$ is generated by $s_{2}, r \gamma_{L / 2}^{x}, \gamma^{y}$ and $q$. In the hydrodynamic eigenspace the symmetries $s_{2}$ and $\gamma^{y}$ act non-trivially, and in the magnetic eigenspace the action of the symmetries $s_{2}, q$ and $\gamma^{y}$ is non-trivial. Hence, the results of Golubitsky et al. (1988) (Section XX §2) on Hopf / steady-state mode interaction with the $\mathbf{O}(2)$ symmetry group are applicable. (The symmetry $q$ plays no rôle, since, in the notation ibid., $q=(\pi, \pi)$ is a superposition of two shifts, in $\phi=\pi$ and $\theta=\pi$, which are elements of $\mathbf{S O}(2)$ and $\mathbf{S}^{1}$, respectively. In our problem, the action of $\mathbf{S O}(2)$ is generated by the shifts $\gamma^{y}$ along the direction of the rolls.) According to Golubitsky et al. (1988), the trivial steady state $\left(\mathrm{R}_{1}\right.$ in our case) bifurcates with emergence of an $s_{2}$-symmetric steady state, when a real eigenvalue becomes positive, and with a simultaneous emergence of rotating (i.e. travelling, in our parlance), TW, and standing waves, SW, when a complex pair of eigenvalues crosses the imaginary axis. The rotating wave can further bifurcate to a modulated rotating wave (which in fact is a 2 -torus).

In our system, in agreement with this general theory, $\mathrm{R}_{1}$ bifurcates to TW (in the hydrodynamic subspace) and to $\mathrm{S}_{2}^{\mathrm{R} 1}$. TW further bifurcates to a modulated travelling wave, $\mathrm{P}_{2}^{\mathrm{TW}}$ (which we classify as a 
Table 5: Families of attractors $\mathrm{F}^{\mathrm{TW}}$ and $\mathrm{C}^{\mathrm{SW}}$. Column 2 presents the type of attractors (disregarding drift frequencies): $\mathrm{P}$ periodic, Q quasiperiodic, $\mathrm{C}$ chaotic, column 3 basic frequencies of periodic and quasiperiodic regimes, columns 5 and 6 time-averaged kinetic and magnetic energies, respectively, the last column the list of individual runs as pairs $T a($ integration time).

\begin{tabular}{|c|c|c|c|c|c|c|c|}
\hline $\begin{array}{l}\text { Fa- } \\
\text { mily }\end{array}$ & Type & \multicolumn{2}{|c|}{$\begin{array}{c}\text { Basic } \\
\text { frequencies }\end{array}$} & $\begin{array}{c}\text { Interval of } \\
\text { existence }\end{array}$ & $\overline{E_{k}}$ & $E_{m}$ & $\begin{array}{l}\text { Individual } \\
\text { runs }\end{array}$ \\
\hline \multirow[t]{9}{*}{$\mathrm{F}^{\mathrm{TW}}$} & $\mathrm{P}_{2}^{\mathrm{TW}}$ & $2.86-2.87$ & & {$[36,57]$} & $234.10-235.93$ & $9.43-11.82$ & $\begin{array}{c}36(351), 41(22), \\
45(29), 48(59), \\
54(251), 57(1274)\end{array}$ \\
\hline & $\mathrm{Q}$ & $2.87-2.94$ & $0.209-0.151$ & {$[60,68]$} & $235.95-235.11$ & $12.01-12.20$ & $\begin{array}{l}60(337), 63(370), \\
65(2378), 68(352)\end{array}$ \\
\hline & $\mathrm{Q}$ & 2.93 & 0.071 & 70 & 235.24 & 12.03 & $70(1646)$ \\
\hline & Q & 2.93 & 0.034 & 71.5 & 235.20 & 12.05 & $71.5(2022)$ \\
\hline & $\mathrm{Q}$ & 2.93 & 0.016 & 72 & 235.16 & 12.08 & $72(1696)$ \\
\hline & $\mathrm{C}$ & & & {$[73,74]$} & $234.76-234.60$ & $11.91-11.81$ & $73(1032), 74(2620)$ \\
\hline & $\mathrm{Q}$ & $2.92-2.94$ & $0.035-0.030$ & $75,77]$ & $234.05-233.31$ & $11.86-11.30$ & $75(457), 77(2413)$ \\
\hline & Q & 2.91 & 0.015 & $78,78.5]$ & $233.90-233.62$ & $11.62-11.60$ & $78(2602), 78.5(1427)$ \\
\hline & $\mathrm{Q}$ & 2.91 & $0.069-0.061$ & {$[79,80]$} & $232.62-232.74$ & $11.04-11.25$ & $79(223), 80(1784)$ \\
\hline \multirow[t]{11}{*}{$\mathrm{C}^{\mathrm{SW}}$} & $\mathrm{C}$ & & & 78 & 236.55 & 17.00 & $78(2796)$ \\
\hline & $\mathrm{Q}$ & 2.86 & $0.048-0.051$ & {$[80,81]$} & $236.76-236.86$ & $16.63-16.62$ & $80(1681), 81(1379)$ \\
\hline & $\mathrm{C}$ & & & {$[82,84]$} & $237.35-237.91$ & $16.68-16.49$ & $\begin{array}{c}82(2558), 83(3174), \\
84(1950)\end{array}$ \\
\hline & $\mathrm{Q}$ & 2.90 & 0.017 & 85 & 237.83 & 16.39 & $85(4052)$ \\
\hline & $\mathrm{C}$ & & & {$[87,96.5]$} & $238.27-240.01$ & $16.22-15.01$ & $\begin{array}{c}87(1812), 90(2851), \\
95(3582), 96(953) \\
96.5(1379)\end{array}$ \\
\hline & $\mathrm{Q}$ & 2.93 & $0.016-0.021$ & {$[97,98]$} & $241.13-240.94$ & $14.52-14.58$ & $97(928), 98(2511)$ \\
\hline & $\mathrm{C}$ & & & {$[99,100]$} & $239.86-239.85$ & $15.02-15.05$ & $99(1682), 100(1999)$ \\
\hline & $\mathrm{Q}$ & 2.88 & 0.026 & {$[101,102]$} & $239.98-240.05$ & $14.91-14.83$ & $101(979), 102(3607)$ \\
\hline & Q & 2.88 & $0.052-0.056$ & {$[103,105]$} & $240.12-240.38$ & $14.73-14.41$ & $103(1339), 105(3847)$ \\
\hline & $\mathrm{P}$ & $2.90-2.89$ & & {$[106,119]$} & $239.59-239.93$ & $13.42-13.04$ & $\begin{array}{c}106(1177), 107(199), \\
110(511), 115(571), \\
117.5(3768), 119(1181)\end{array}$ \\
\hline & $\mathrm{P}^{\mathrm{SW}}$ & $2.89-2.88$ & & {$[120,126]$} & $239.82-239.90$ & $13.03-13.31$ & $\begin{array}{c}120(675), 121.5(517) \\
123(411), 126(1002)\end{array}$ \\
\hline
\end{tabular}


periodic orbit, since we omit drift frequencies in the description of attractors). At $T a=87$ the bifurcation where $\mathrm{P}_{2}^{\mathrm{TW}}$ emerges is subcritical, and close to the point of bifurcation the periodic orbit is unstable. It gains stability as it turns back in a saddle-node bifurcation at $T a=36$. We classify the stable periodic orbit observed in computations as the modulated rotating wave predicted by the theory, because 1) of the similarity of the spatial structure of TW and the flow in $\mathrm{P}_{2}^{\mathrm{TW}}$, as well as of the dominant magnetic mode of TW and magnetic field in $\mathrm{P}_{2}^{\mathrm{TW}} ; 2$ ) the symmetry group of $\mathrm{P}_{2}^{\mathrm{TW}}$ is the one expected on the theoretical grounds for the branch emerging at $T a=87$ from TW due to the mode interaction (note that it includes a spatio-temporal symmetry: the symmetry about a vertical axis with a shift in time by a half of a period); and 3) the temporal frequency of $\mathrm{P}_{2}^{\mathrm{TW}}$ is close to the Hopf frequency of TW.

The steady state $\mathrm{S}_{2}^{\mathrm{R} 1}$ bifurcates as $T a$ is decreased to a less symmetric steady state $\mathrm{S}_{1}^{\mathrm{R} 1}$, which is outside the Hopf / steady-state mode interaction center manifold. As Ta is decreased further, $\mathrm{S}_{1}^{\mathrm{R} 1}$ becomes unstable in a Hopf bifurcation with a stable periodic orbit emerging. We label it $\mathrm{P}^{\mathrm{SW}}$, because its spatial structure and temporal frequency are similar to those of the unstable standing wave $\mathrm{SW}$ emerging from $\mathrm{R}_{1}$ simultaneously with the travelling wave TW, and its symmetry group is a subgroup of the one of SW. The general theory of Hopf / steady-state mode interaction predicts two types of periodic orbits bifurcating from SW, but judging by its symmetries none of them is our $\mathrm{P}^{\mathrm{SW}}$. The conjectured relation of $\mathrm{P}^{\mathrm{SW}}$ with $\mathrm{SW}$ is shown by thin dashed lines on Fig. 12 .

\subsection{MHD attractors emerging from TW, $36<T a \leq 127$}

Both periodic orbits, $\mathrm{P}_{2}^{\mathrm{TW}}$ and $\mathrm{P}^{\mathrm{SW}}$, give rise to complex families of attractors, $\mathrm{F}^{\mathrm{TW}}$ and $\mathrm{C}^{\mathrm{SW}}$, discussed in this subsection (see Table 5). Like $\mathrm{P}_{1}^{\mathrm{TW}}$, all attractors constituting $\mathrm{F}^{\mathrm{TW}}$ drift along both horizontal axes and thus have two drift frequencies. The family $\mathrm{F}^{\mathrm{TW}}$ starts from the primary periodic MHD attractor $\mathrm{P}_{2}^{\mathrm{TW}}$ at $T a=36$.

As $T a$ is increased beyond $T a \approx 60$, the second frequency, $f_{2}$, appears in a Hopf bifurcation, i.e. a stable torus emerges. Afterwards, a sequence of bifurcations of halving of $f_{2}$ takes place (often such a sequence is called a cascade of period doubling bifurcations for a torus; we prefer to abstain from the use of this terminology, since the concept of a period applied to a torus is not all too transparent). This sequence of bifurcations is analogous to the Feigenbaum (1978) scenario of period doublings for a logistic map, and we label this family of attractors by $\mathrm{F}$.

Logistic map is defined by the recurrent relation $x_{n+1}=r x_{n}\left(1-x_{n}\right), 0<x<1$. When $r$ is increased over 3, a stable 2-cycle is created in a flip bifurcation. Subsequent flip bifurcations result in emergence of cycles of lengths $4,8, \ldots$ The bifurcations accumulate at $r=3.57$. For larger $r$, chaotic behaviour sets in, alternating with windows of periodic orbits of periods $(2 m+1) 2^{k}$. For $r>4, x_{n}$ escapes from the interval $0<x<1$ and diverges.

In the convective hydromagnetic system, on increasing $T a$ we have detected three halvings of the frequency $f_{2} \approx 0.15$, followed by a chaotic behaviour. For larger $T a$, lower basic frequencies set in, $f_{2} / 3$ for $75 \leq T a \leq 77$ and $f_{2} / 6$ for $78 \leq T a \leq 78.5$; their appearance agrees with the general theory. No more halvings have been observed. This is consistent with the fact that a complete sequence of period doubling bifurcations of tori is a structurally unstable scenario (see Coullet, 1984), and generically it is interrupted by the onset of chaos. (We could also miss some halvings just having considered not enough values of $T a$.) For still larger $T a$, a frequency $\tilde{f}_{2} \approx f_{2} / 2$ is observed. On the one hand, emergence of this quasiperiodic regime is clearly outside the framework of the Feigenbaum period doubling scenario (cf. Fig. 13 (b) and (h) ), suggesting that attractors for $79 \leq T a \leq 80$ do not belong to our Feigenbaum family. On the other, the standard theory takes into account only two first terms in the Taylor expansion of the dynamical system on the central manifold, and terms of higher order may be responsible for the birth of this new regime.

Plots of the energies (Fig. 13), not affected by drift frequencies, clearly display the frequency halving. By contrast, plots of the time dependencies of real or imaginary parts of individual Fourier coefficients (not shown) are marred by the extra drift frequencies, making these plots by far more obscure. A similar effect is observed in Poincaré sections. Frequency halving for tori usually shows itself unambiguously on Poincaré sections, however, the standard Poincaré sections are not particularly enlightening when additional drift frequencies are present. Their influence is eliminated in Poincaré sections, which are constructed with the use of absolute values of Fourier coefficients (cf. Figs. 14] and 15). 


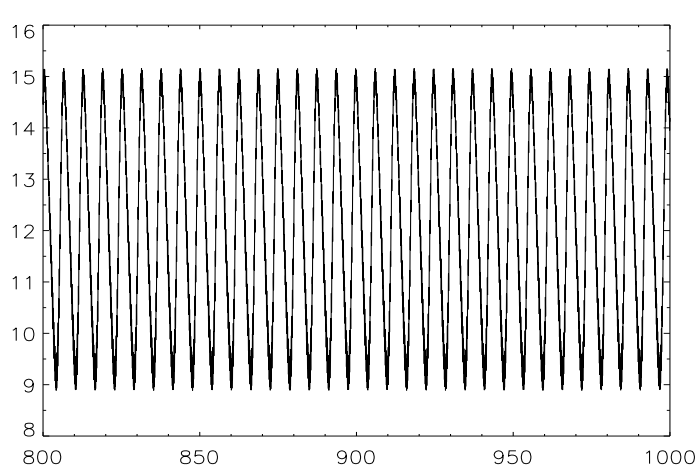

(a)

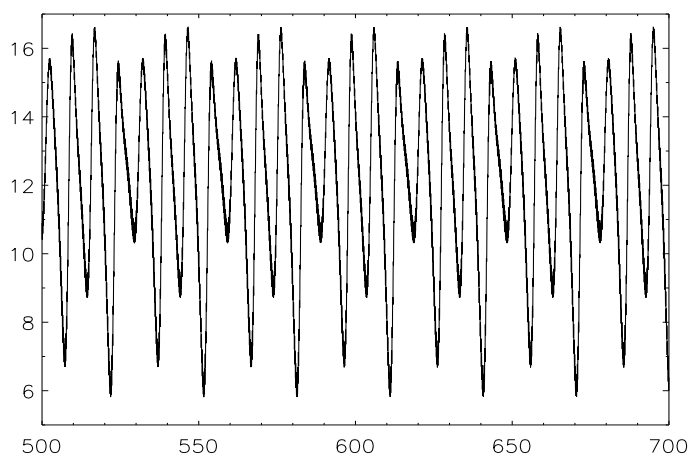

(c)

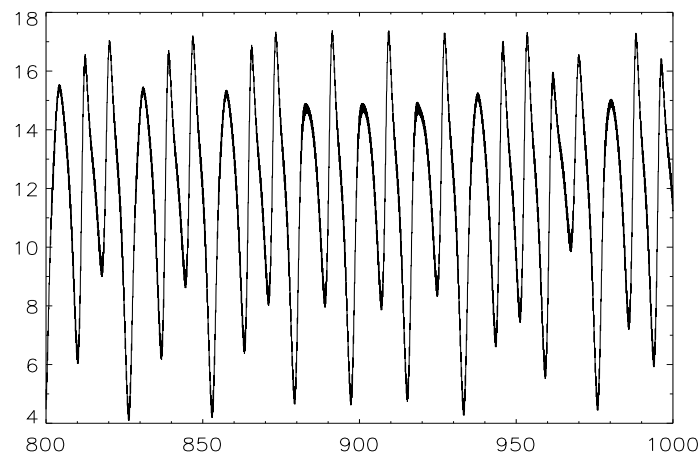

(e)

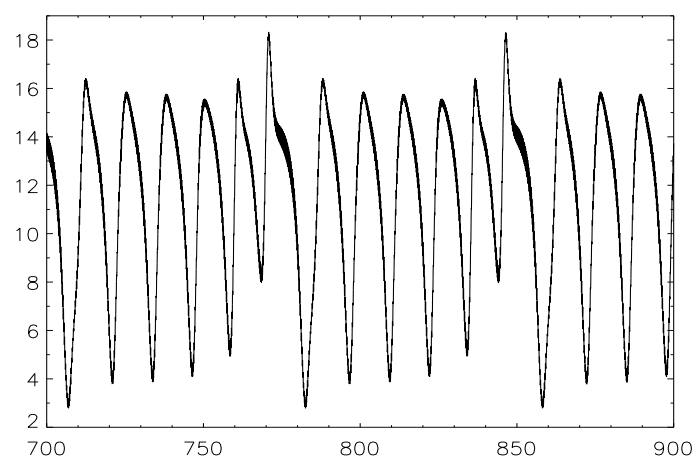

(g)

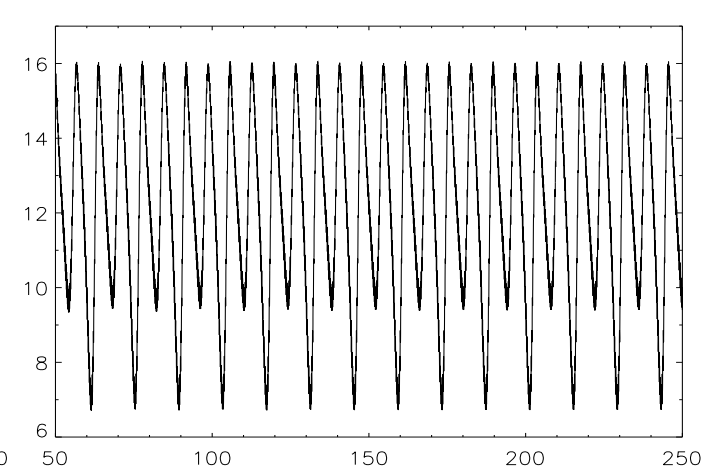

(b)

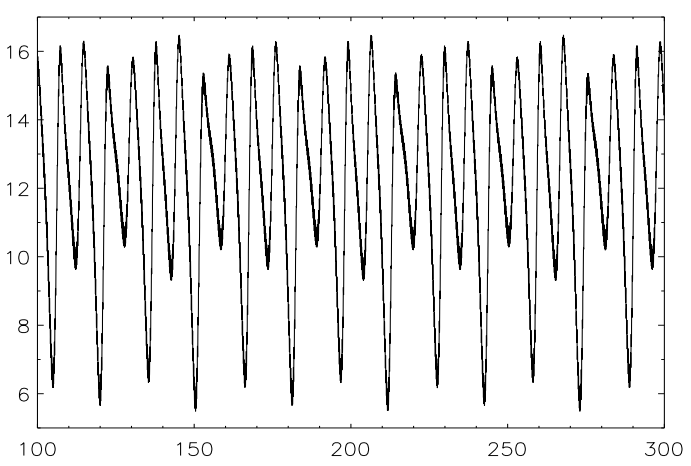

(d)

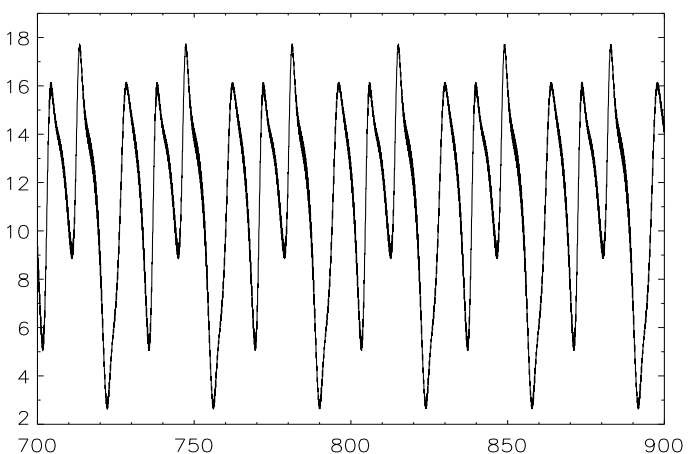

(f)

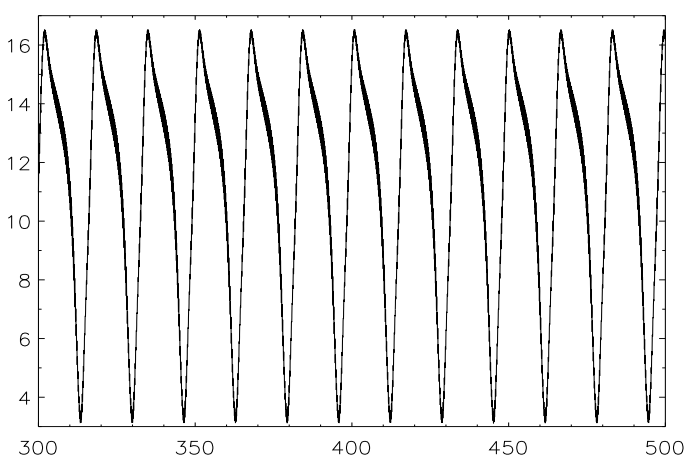

(h)

Figure 13: Magnetic energy (vertical axis) versus time (horizontal axis) for $\mathrm{F}^{\mathrm{TW}}$ : $T a=65$ (a), $\mathrm{Ta}=70$ (b), $T a=71.5$ (c), $T a=72(\mathrm{~d}) T a=74(\mathrm{e}), T a=77$ (f), $T a=78.5(\mathrm{~g}), T a=80(\mathrm{~h})$. 


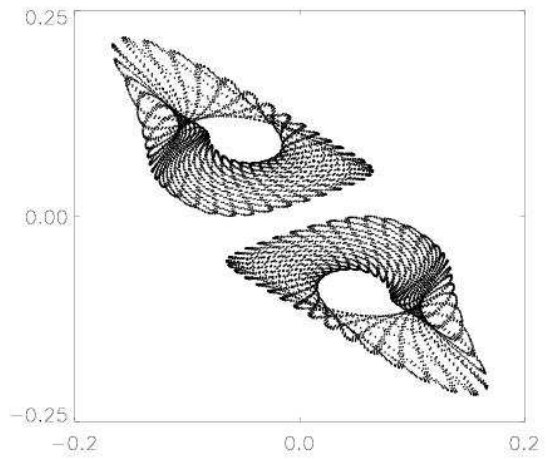

(a)

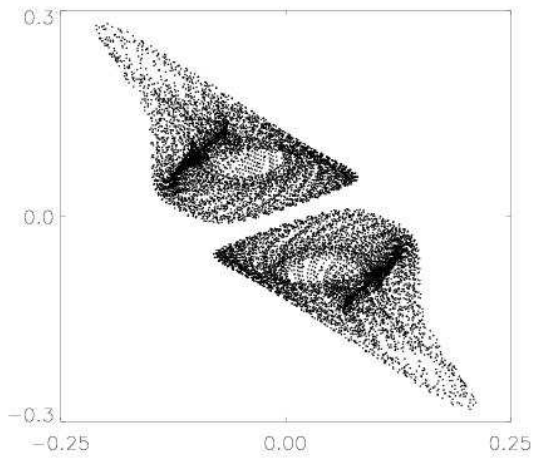

(d)

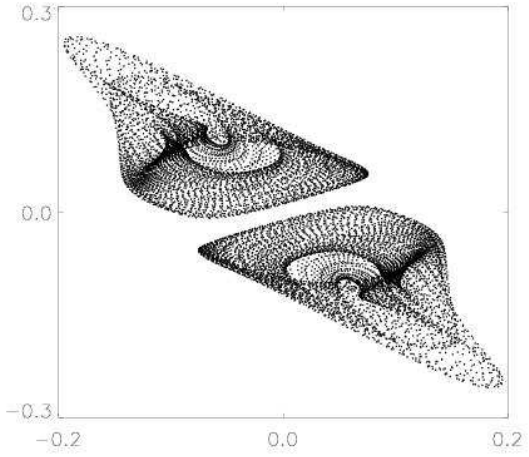

(b)

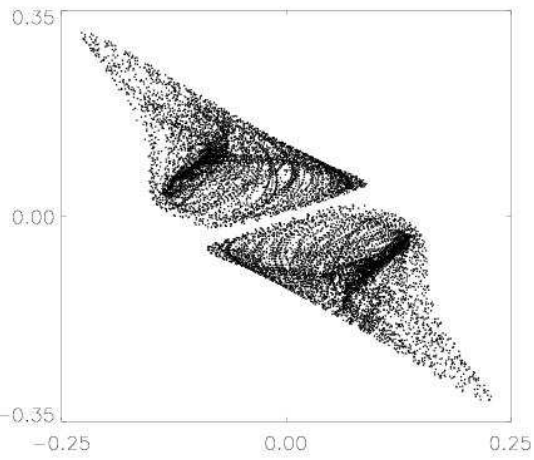

(e)

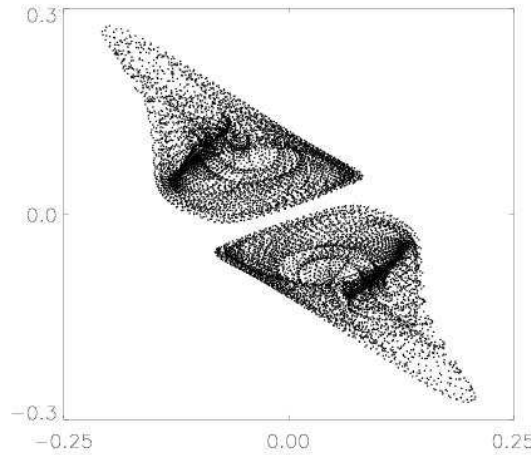

(c)

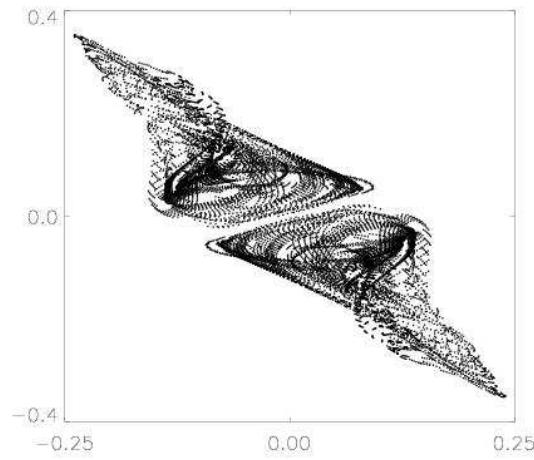

(f)

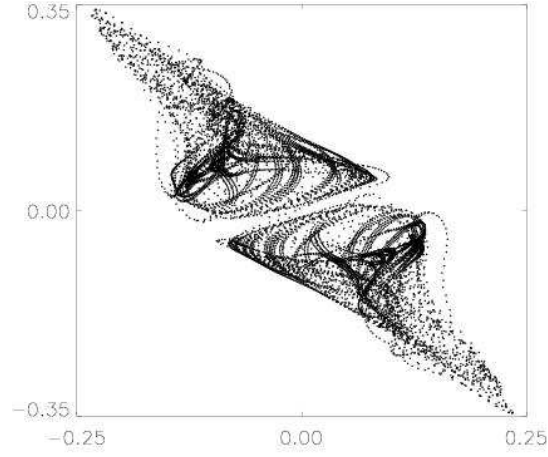

(g)

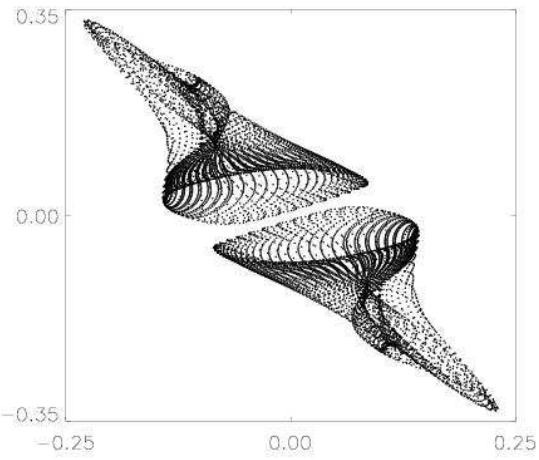

(h)

Figure 14: Poincaré sections of regimes in the family $\mathrm{F}^{\mathrm{TW}}$ on the $\left(\operatorname{Re} \hat{v}_{-1,2,1}^{x}, \operatorname{Re} \hat{v}_{-1,2,1}^{y}\right)$ plane (horizontal and vertical axes, respectively) defined by the condition $\operatorname{Re} \hat{v}_{-1,2,1}^{z}=0: T a=65$ (a), $T a=70$ (b), $T a=71.5$ (c), $T a=72$ (d), $T a=74$ (e), $T a=77$ (f), $T a=78.5$ (g), $T a=80$ (h). 


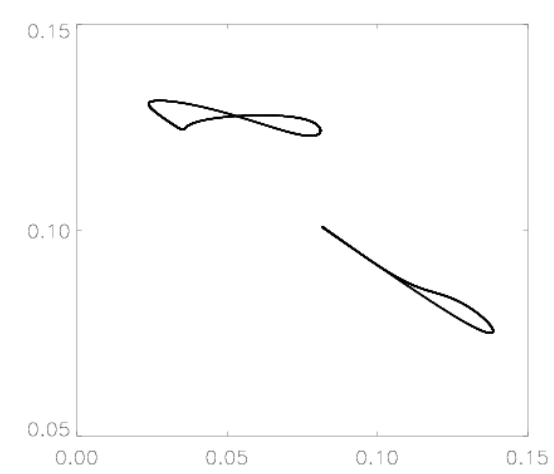

(a)

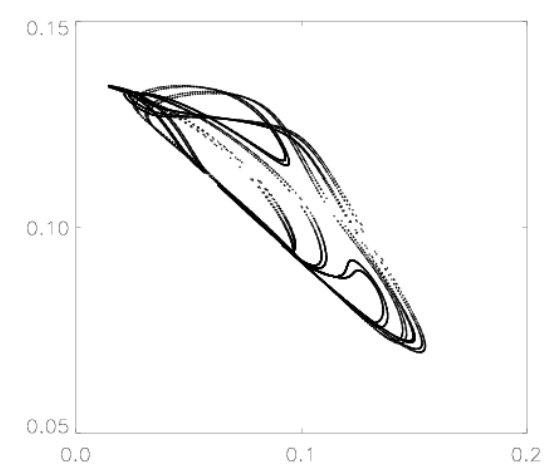

(d)

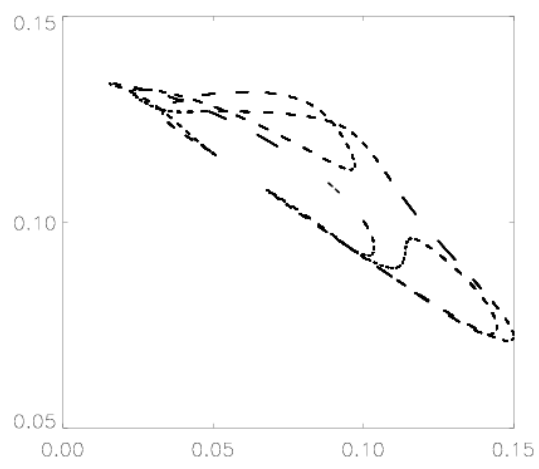

(b)

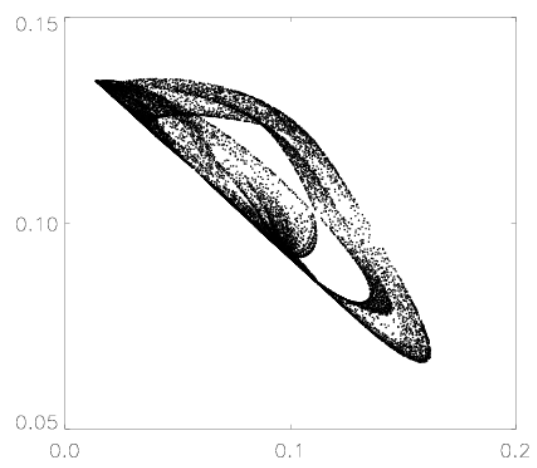

(e)

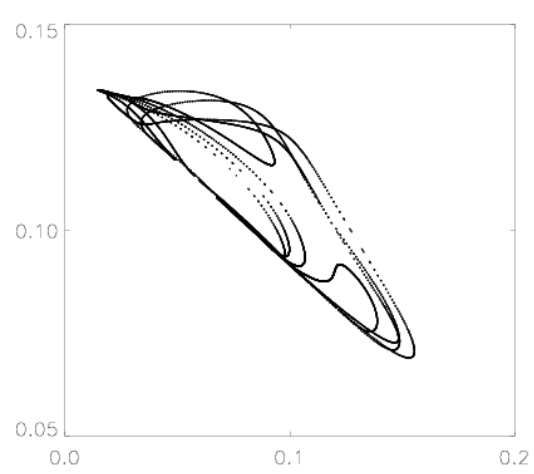

(c)

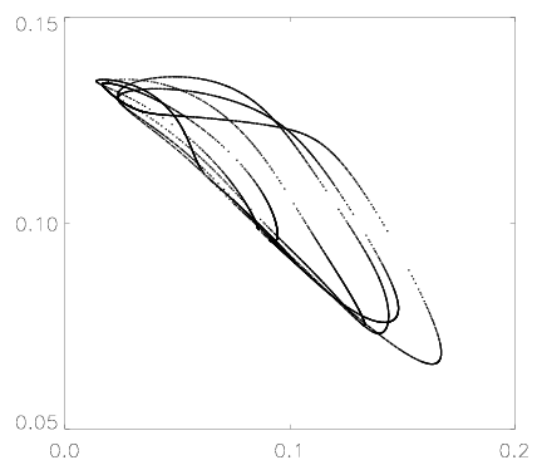

(f)

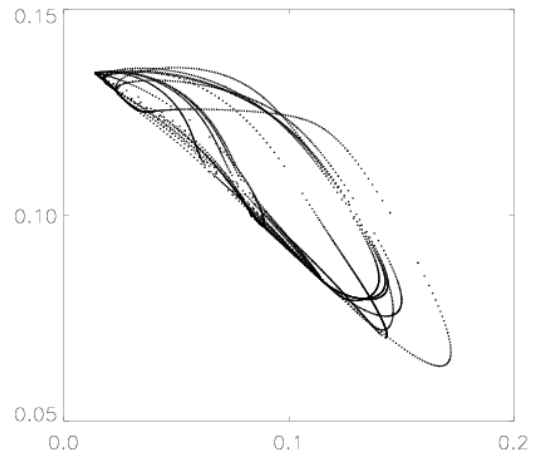

(g)

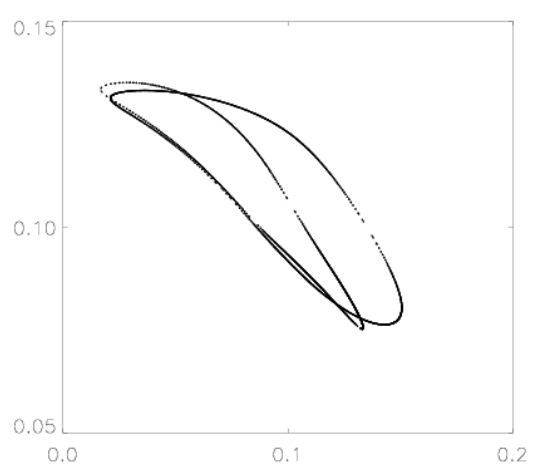

(h)

Figure 15: Poincaré sections for regimes in the family $\mathrm{F}^{\mathrm{TW}}$ on the $\left(\left|\hat{v}_{-1,2,1}^{x}\right|,\left|\hat{v}_{-1,2,1}^{y}\right|\right)$ quadrant (horizontal and vertical axes, respectively) defined by the condition $\left|\hat{v}_{-1,2,1}^{z}\right|=0.2: T a=65$ (a); Ta=70 (b); Ta=71.5 (c); Ta=72(d); Ta=74 (e); $T a=77$ (f); $T a=78.5$ (g); $T a=80$ (f). 


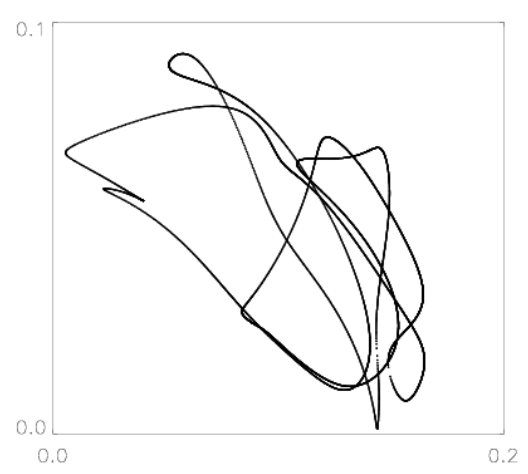

(a)

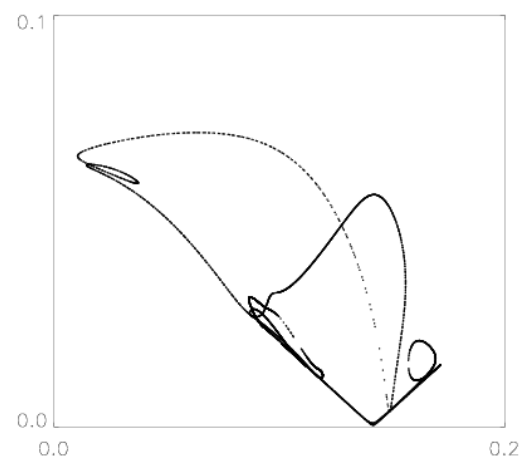

(d)

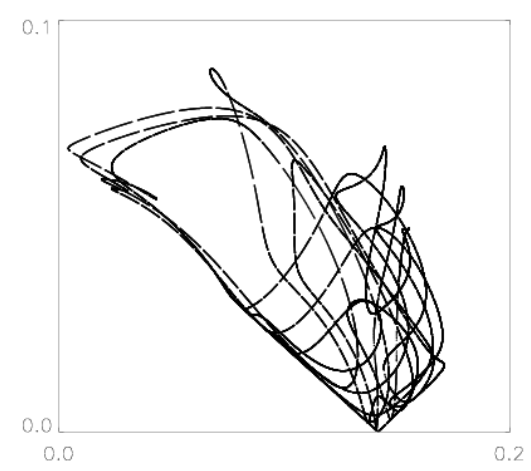

(b)

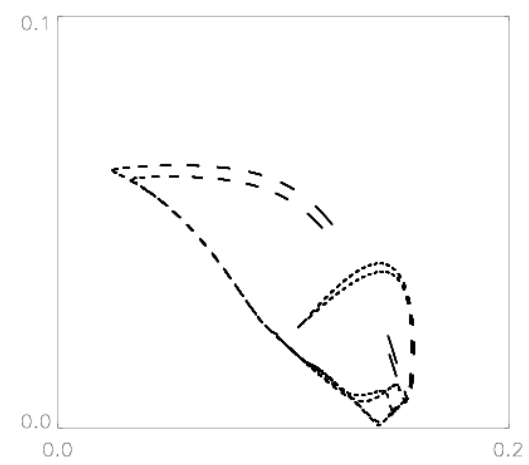

(e)

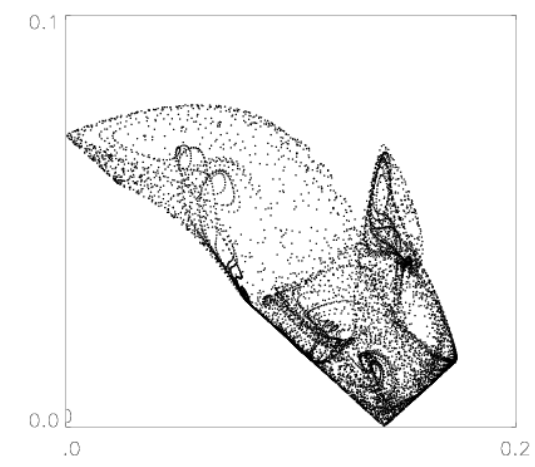

(c)

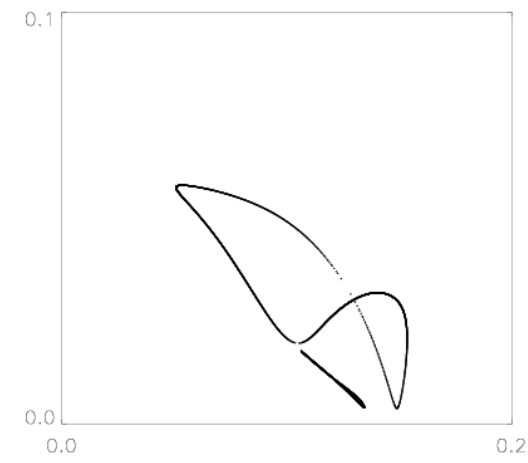

(f)

Figure 16: Poincaré sections for regimes in the family $\mathrm{C}^{\mathrm{SW}}$ on the $\left(\left|\hat{v}_{-1,2,1}^{x}\right|,\left|\hat{v}_{-1,2,1}^{y}\right|\right)$ quadrant (horizontal and vertical axes, respectively) defined by the condition $\left|\hat{v}_{-1,2,1}^{z}\right|=0.1: T a=80$ (a); $T a=85$ (b); $T a=90$ (c); $T a=98$ (d); $T a=102$ (e); $T a=105$ (f).

The family $\mathrm{C}^{\mathrm{SW}}$ exists for $78 \leq T a \leq 126$. It is comprised of chaotic attractors, alternating with windows of periodic and quasiperiodic regimes (Fig. 16). The family starts with the periodic regime $\mathrm{P}^{\mathrm{SW}}$, which is stable for $120 \leq T a \leq 126$. The orbit possesses a symmetry, which is a combination of $s_{2}$ with a shift by a half of its temporal period, hence, in agreement with Krupa (1990), the periodic orbit does not drift. As $T a$ is decreased, the symmetry is lost, the emerging non-symmetric orbit and subsequent attractors of the family have two drifting frequencies (not discussed here). Subsequently, a second frequency appears in a Hopf bifurcation, which is halved afterwards. On a further decrease of $T a$, we observe an intermittency of chaotic and quasiperiodic attractors. The sequence "a periodic orbit, a torus, chaos" is standard; it is usually explained by appearance of the third basic frequency, which makes the system structurally unstable and results in the onset of a chaotic behaviour (Ruelle and Takens, 1971). The observed windows of quasiperiodicity can be attributed to frequency locking (see, e.g., Ott, 2002).

Notably, coexistence of attractors of different types is observed: two types of MHD attractors $\left(\mathrm{F}^{\mathrm{TW}}\right.$ and $\mathrm{P}_{1}^{\mathrm{TW}}$ ) in the interval $36 \leq T a \leq 81$, and a (magnetically stable) hydrodynamic attractor (TW) with an MHD attractor $\left(\mathrm{C}^{\mathrm{SW}}\right)$ in the interval $81.80<T a<87$. Coexistence of a hydrodynamic and MHD attractors can be described in physical terms, as stiff excitation of a magnetic field: small magnetic perturbations of TW decay to the hydrodynamic state, while large magnetic perturbations give rise to MHD regimes. Furthermore, in the interval $78 \leq T a \leq 80$ three MHD attractors coexist: $\mathrm{P}_{1}^{\mathrm{TW}}, \mathrm{F}^{\mathrm{TW}}$ and $\mathrm{C}^{\mathrm{SW}}$. From the point of view of the theory of dynamical systems there is nothing extraordinary in coexistence of attractors in a nonlinear system, however, to the best of our knowledge coexistence of three convective MHD attractors was never observed before. 


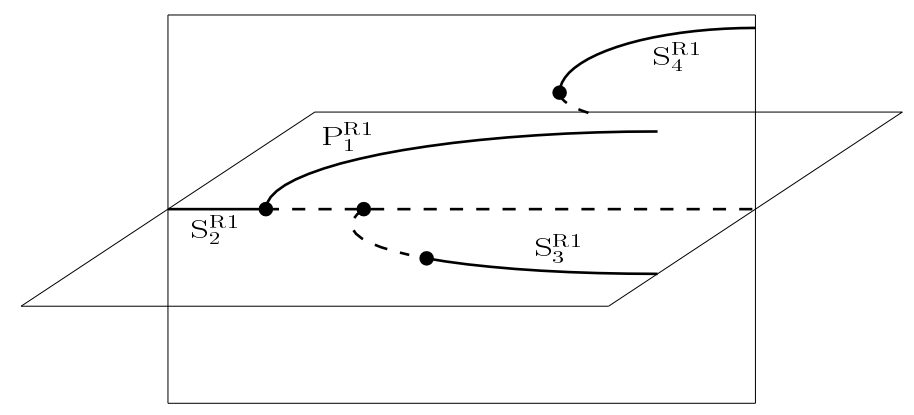

Figure 17: Phase portrait of the MHD system on the interval $216<T a<224$.

\subsection{MHD attractors emerging from $\mathrm{R}_{1}, 127 \leq \mathrm{Ta}<506$}

Dominant magnetic modes of $\mathrm{R}_{1}$ are of the same type for all $T a$. As attested by symmetries, the primary MHD attractor is the steady state $\mathrm{S}_{2}^{\mathrm{R} 1}$ detaching from $\mathrm{R}_{1}$ at $T a=725.3$ in a subcritical pitchfork bifurcation. It exists (i.e. the MHD states are stable) for $170 \leq T a \leq 216$. On decreasing $T a$, a steady state $\mathrm{S}_{1}^{\mathrm{R} 1}$ with a smaller group of symmetries emanates in a pitchfork bifurcation; it becomes unstable and bifurcates to $\mathrm{P}_{2}^{\mathrm{TW}}$ in a Hopf bifurcation.

On increasing $T a$, in a small interval $216<T a<224$ four bifurcations occurring in the vicinity of $\mathrm{S}_{2}^{\mathrm{R} 1}$ (in the phase space) are observed (see Fig. $7(\mathrm{~b})$ ). They take place in two distinct invariant subspaces, Fix $\left(r s_{2}\right)$ and $\operatorname{Fix}\left(s_{2}\right)(\operatorname{Fix}(s)$ denotes the set of fixed points under the action of a symmetry $s)$. Developments in each invariant subspace are mutually independent (see a sketch of geometry of the phase space shown on Fig. 17 the vertical plane represents $\operatorname{Fix}\left(r s_{2}\right)$ and the horizontal one $\left.\operatorname{Fix}\left(s_{2}\right)\right)$.

The first (on increasing $T a$ ) one is a Hopf bifurcation in $\operatorname{Fix}\left(r s_{2}\right)$ at $T a=216.13$, in which a periodic orbit, $\mathrm{P}_{1}^{\mathrm{R} 1}$, emanates. Another one is a saddle-node bifurcation at $T a=223.1$ in the same invariant subspace, in which a branch of steady states, $\mathrm{S}_{4}^{\mathrm{R} 1}$, terminates. Near the point of bifurcation the basin of attraction of the steady states becomes vanishingly small, requiring short steps when continuing the branch in $T a$ so that computed trajectories were not attracted by $\mathrm{P}_{1}^{\mathrm{R} 1}$. On decreasing $T a$ from, say, 224, eigenvalues of stability modes are in the beginning imaginary, but both the real and imaginary parts decrease in magnitude, apparently tending to zero. At $T a=T a_{c}, 223.101<T a_{c}<223.10075$, the discriminant of the quadratic characteristic equation, defining the two eigenvalues, changes its sign. As a result, the dominant eigenvalue exhibits a counterintuitive behaviour: Coefficients of the characteristic equation depend almost linearly on $T a$ near this value $T a_{c}$, but the change of sign of the discriminant implies, that the dominant real eigenvalue is continuous, but non-smooth (see Fig. 18). The graph of the growth rate of the dominant mode bends at $T a_{c}$ and for $T a<T a_{c}$ behaves like $\sqrt{T a_{c}-T a}$. The largest real eigenvalue starts to grow much faster, and a saddle-node bifurcation occurs between $T a=223.09999$ and 223.1. An almost simultaneous vanishing of both real and imaginary parts of eigenvalues implies, that two parameters are necessary to describe this bifurcation, i.e. we are in a vicinity of a codimension two bifurcation. Variation of $T a$ near the left end of the branch $\mathrm{S}_{4}^{\mathrm{R} 1}$ is equivalent to a motion along an one-dimensional curve on the plane of the parameters.

Vanishing of a pair of complex eigenvalues of linearisation of a dynamical system is an attribute of the Takens-Bogdanov bifurcation (see Guckenheimer and Holmes, 1988). There are further indications that the bifurcation occurring in $\operatorname{Fix}\left(r s_{2}\right)$ close (in the parameter space) to the values, which are fixed in our simulations, might be a Takens-Bogdanov bifurcation: in this interval, $216<T a<224$, a periodic orbit emerges and afterwards becomes unstable or disappears and a steady state emerges, and they are close neighbours in the same symmetric subspace. However, a more attentive inspection of the system convinces that this conjecture is wrong.

Only $\mathrm{S}_{2}^{\mathrm{R} 1}$ might be the trivial steady state suffering the bifurcation (although the behaviour of the eigenvalues reminiscent of the Takens-Bogdanov bifurcation is registered for $\mathrm{S}_{4}^{\mathrm{R} 1}$, this branch turns back in a saddle-node bifurcation and thus can not serve as the trivial steady state, existing in the case of TakensBogdanov bifurcation for all parameter values in the vicinity of the point of bifurcation). It is quite possible that a pair of complex eigenvalues of linearisation of $\mathrm{S}_{2}^{\mathrm{R} 1}$ simultaneously become zero upon a variation 


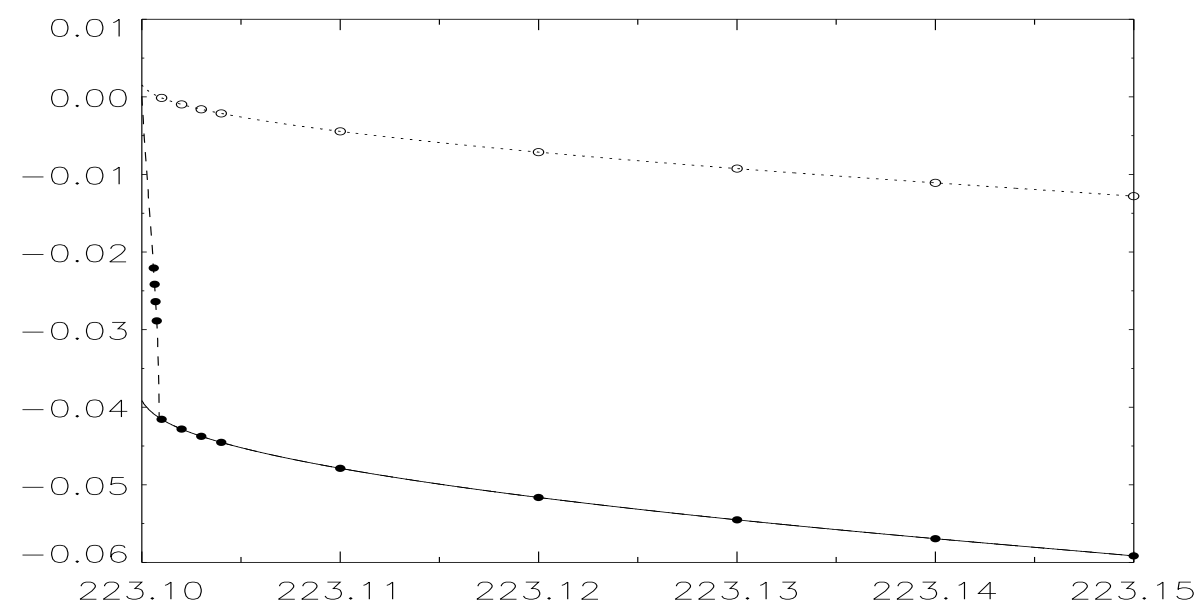

Figure 18: Computed mean of the two dominant eigenvalues (black circles; coincides with the real part of the eigenvalues for $T a>T a_{c} \approx 223.101$, where the two eigenvalues are complex conjugate) and the discriminant of the quadratic characteristic equation (open circles) for the dominant eigenvalues of the operator of linearisation versus Ta (horizontal axis) near the left end of the interval of existence of $\mathrm{S}_{4}^{\mathrm{R} 1}$. Padé extrapolation of the mean of the two eigenvalues (solid line), the discriminant of the characteristic equation (dotted line) and the resultant extrapolated dominant real eigenvalue for $T a<T a_{c}($ dashed line).

of $T a$ and a second parameter: $\mathrm{S}_{2}^{\mathrm{R} 1}$ does have small in magnitude complex eigenvalues in the interval $216 \leq T a \leq 220$ for the parameter values that we have employed; also it is not far from $\mathrm{S}_{4}^{\mathrm{R} 1}$, suggestive of a similar behaviour of eigenvalues of the two branches. The structure of our system is compatible only with the Takens-Bogdanov bifurcation (with the $\mathbf{Z}_{2}$ symmetry group generated by $s_{2}$, which $\mathrm{S}_{4}^{\mathrm{R} 1}$ does not possess) involving a stable periodic orbit and stable steady states, distinct from the trivial one; such a diagram is shown on Figs. 7.3.7 and 7.3.9 ibid. On these Figures, the trivial steady state (the analogue of $\mathrm{S}_{2}^{\mathrm{R} 1}$ ) is stable for $\mu_{1}<0, \mu_{2}<0$ (using the parameter notation ibid.), the other two steady states (the analogues of $\mathrm{S}_{4}^{\mathrm{R} 1}$ and its symmetric counterpart) for $\mu_{1}>\max \left(0, \mu_{2}\right)$, and a periodic orbit for $\mu_{2}>\max \left(0, c \mu_{1}\right), c \approx 0.752$. Hence variation of $T a$ on the interval $216 \leq T a \leq 224$ must be equivalent to a motion along a curve on the $\left(\mu_{1}, \mu_{2}\right)$ plane, beginning in the quadrant $\mu_{1}<\overline{0}, \mu_{2}<0$, following to the quadrant $\mu_{1}<0, \mu_{2}>0$, and passing to the region $\mu_{1}>0, \mu_{2}<c \mu_{1}$. Consequently, the curve must cross the line $\mu_{1}=0$, where in the Takens-Bogdanov bifurcation a branch of steady states (analogues of $\mathrm{S}_{4}^{\mathrm{R} 1}$ ), stable or unstable depending on the sign of $\mu_{1}$, emanates from the trivial steady state in a pitchfork bifurcation. This is inconsistent with the behaviour of eigenvalues of linearisation of $\mathrm{S}_{2}^{\mathrm{R} 1}$ (we have computed these unstable steady states after the Hopf bifurcation, giving rise to $\mathrm{P}_{1}^{\mathrm{R} 1}$, by imposing the symmetries $r \gamma_{L / 2}^{x}$ and $s_{2}$ ) - the dominant and subdominant eigenvalues do become real between $T a=221$ and 221.5 , but they remain positive and do not become small on the interval $221.5 \leq T a \leq 224$. Therefore we conclude, that bifurcations in our system are not induced by a Takens-Bogdanov bifurcation (a finite perturbation of a bifurcation of this type, in which more than two parameters are essential for the description of the bifurcation, is not ruled out). Further analysis is necessary to understand it theoretically.

At $T a=216.75$ an eigenvalue of linearisation of (1) near $\mathrm{S}_{2}^{\mathrm{R} 1}$ with the associated eigenvector in $\operatorname{Fix}\left(s_{2}\right)$ (the dominant eigenvalue of the restriction of the operator of linearisation to this subspace) becomes positive. In this supercritical pitchfork bifurcation a steady state $\mathrm{S}_{3}^{\mathrm{R} 1}$ emerges. Since at the bifurcation $\mathrm{S}_{2}^{\mathrm{R} 1}$ is unstable with respect to perturbations in $\operatorname{Fix}\left(r s_{2}\right)$, the branching steady state is also unstable to such perturbations near the point of bifurcation. $\mathrm{S}_{3}^{\mathrm{R} 1}$ gains stability at $T a=218.64$ in a subcritical Hopf bifurcation. The branch is stable up to a subcritical pitchfork bifurcation at $T a=271.66 . \mathrm{S}_{3}^{\mathrm{R} 1}$ is the only MHD steady state that we have found, whose group of symmetries does not involve superpositions of the symmetry $r$ with any other symmetries. As a result, magnetic patterns on top and bottom of the layer are different (cf. Fig. 11 (c) and other panels on this figure).

We again encounter coexistence of three distinct MHD attractors in the interval $223.11 \leq T a \leq 223.3$ : $\mathrm{S}_{3}^{\mathrm{R} 1}, \mathrm{~S}_{4}^{\mathrm{R} 1}$ and $\mathrm{P}_{1}^{\mathrm{R} 1}$. The steady state $\mathrm{S}_{4}^{\mathrm{R} 1}$ becomes unstable at $\mathrm{Ta}=466.5$ in a subcritical pitchfork bifurca- 


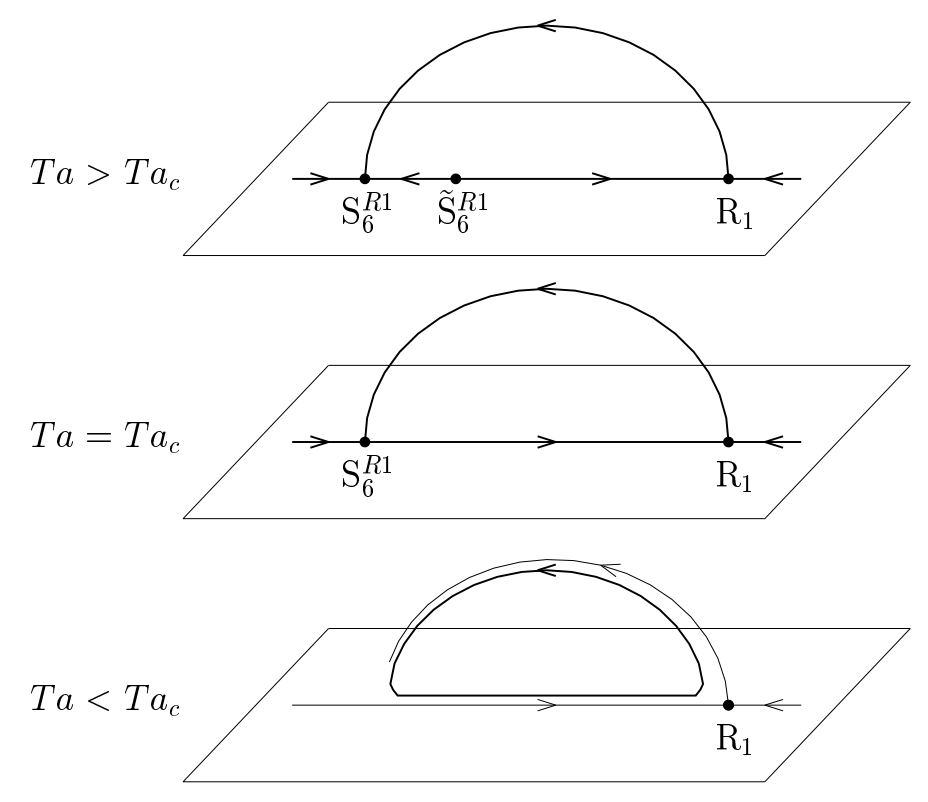

Figure 19: Phase portrait of the MHD system near $T a=T a_{c} \approx 506.07$.

tion.

The symmetry group of $S_{5}^{R 1}$ is a subgroup of the one of $S_{2}^{R 1}$ (see Table 3). That the steady states are related and some symmetries are lacking, is seen on Fig. 11 (b) and (e) displaying magnetic patterns of the steady states. These facts suggest that $\mathrm{S}_{5}^{\mathrm{R} 1}$ bifurcates from the unstable $\mathrm{S}_{2}^{\mathrm{R} 1}$. At both ends of the interval of stability, $\mathrm{S}_{5}^{\mathrm{R} 1}$ undergoes supercritical Hopf bifurcations with stable periodic orbits, $\mathrm{P}_{2}^{\mathrm{R} 1}$ and $\mathrm{P}_{3}^{\mathrm{R} 1}$, emerging. The periodic orbit $\mathrm{P}_{2}^{\mathrm{R} 1}$ with the same group of symmetries as that of $\mathrm{S}_{5}^{\mathrm{R} 1}$ is observed for $343 \leq T a \leq 377$.

The other periodic orbit bifurcating from $\mathrm{S}_{5}^{\mathrm{R} 1}, \mathrm{P}_{3}^{\mathrm{R} 1}$, exists in a small interval $505.1 \leq \mathrm{Ta} \leq 506$ and it has a much smaller symmetry group than $\mathrm{S}_{5}^{\mathrm{R} 1}$.

\subsection{MHD attractors emerging from $\mathrm{R}_{1}$, Ta near 506}

At the right end of the interval of existence of the periodic orbit $\mathrm{P}_{3}^{\mathrm{R} 1}$, it terminates near $T a=506$ on a steady state $\mathrm{S}_{6}^{\mathrm{R} 1}$ in a bifurcation, which appears to be similar to a saddle-node bifurcation on invariant circle (SNIC; see Izhikevich, 2006). However, since the symmetry group of the system is non-trivial, the details of the bifurcation in our case differ from those of the canonical SNIC. In a generic system, a periodic orbit emanates in a SNIC bifurcation subsequent upon a saddle-node bifurcation of a steady state. The SNIC occurs under the condition that for any parameter value before the saddle-node bifurcation (we only consider a small neighbourhood of the critical value of the bifurcation parameter), both parts of the one-dimensional unstable manifold of the unstable steady state terminate on the stable one. At the point of bifurcation, the two steady states collide and a homoclinic trajectory emerges. After the bifurcation, a periodic orbit is formed from this homoclinic trajectory.

SNIC is generic in one-parameter dynamical systems and it is often observed in simulations, where a parameter is varied. At $T a=T a_{c} \approx 506.07$ the periodic orbit $\mathrm{P}_{3}^{\mathrm{R} 1}$ terminates on a heteroclinic cycle; occurrence of this upon variation of just one scalar parameter requires some degeneracy of the system, since a heteroclinic (homoclinic) connection from an equilibrium to an unstable equilibrium in a generic system occurs only for a singular parameter value (such connections are structurally unstable). For formation of a heteroclinic cycle, several such connections must happen simultaneously.

Existence of a heteroclinic orbit at $T a=T a_{c}$ becomes possible due to the presence of a non-trivial symmetry group. Structural stability of homoclinic and heteroclinic connections to an unstable equilibrium in symmetric systems relies on the presence of symmetry-invariant subspaces (Guckenheimer and Holmes, 1988). A sketch of geometry of the phase space of our system is shown on Fig. 19. The steady state $\mathrm{S}_{6}^{\mathrm{R} 1}$ 


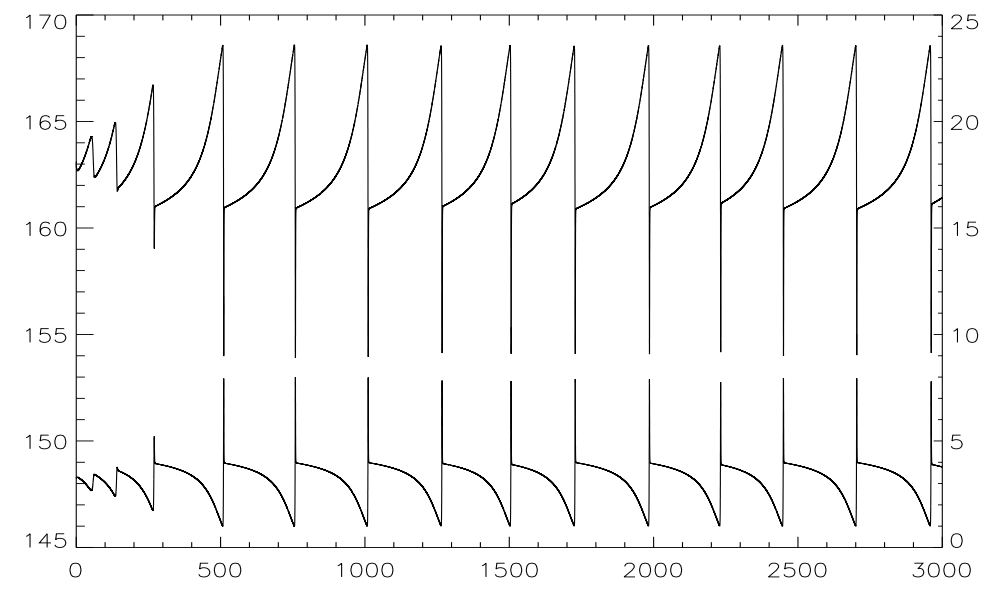

Figure 20: Kinetic (upper curve, left vertical axis) and magnetic (lower curve, right vertical axis) energies for $T a=506, \mathrm{P}_{3}^{\mathrm{R}} 1$ as a function of time (horizontal axis) for a trajectory starting at the steady state $\mathrm{S}_{5}^{\mathrm{R} 1}$, the MHD attractor for $T a=505$.

possesses a symmetry group isomorphic to $\mathbf{D}_{4} \ltimes \mathbf{Z}_{2}$. The plane represents the fixed point subspace for the steady state symmetry group, the vertical direction the antisymmetric complement. We describe the bifurcation starting from larger values of $T a$ slightly above the saddle-node bifurcation of $\mathrm{S}_{6}^{\mathrm{R} 1}$. Denote by $\tilde{\mathrm{S}}_{6}^{\mathrm{R} 1}$ the unstable counterpart of $\mathrm{S}_{6}^{\mathrm{R} 1}$; it belongs to $\operatorname{Fix}\left(\mathbf{D}_{4} \ltimes \mathbf{Z}_{2}\right)$. The one-dimensional unstable manifold of $\tilde{\mathrm{S}}_{6}^{\mathrm{R} 1}$ also belongs to this subspace. A part of the unstable manifold terminates on the stable $\mathrm{S}_{6}^{\mathrm{R} 1}$, another one on $R_{1}$ (this has been checked numerically), which is stable within this subspace. The connections from $\tilde{S}_{6}^{\mathrm{R} 1}$ to $\mathrm{S}_{6}^{\mathrm{R} 1}$ and $\mathrm{R}_{1}$ are structurally stable. The one-dimensional unstable manifold of $\mathrm{R}_{1}$ terminates on the stable $\mathrm{S}_{6}^{\mathrm{R} 1}$ (we have also checked this numerically).

At $T a=T a_{c} \mathrm{~S}_{6}^{\mathrm{R} 1}$ and $\tilde{\mathrm{S}}_{6}^{\mathrm{R} 1}$ collide, thus creating the structurally unstable heteroclinic cycle $\mathrm{S}_{6}^{\mathrm{R} 1} \rightarrow \mathrm{R}_{1} \rightarrow$ $\mathrm{S}_{6}^{\mathrm{R} 1}$ (it exists only for a single value of $T a$ ). For $T a<T a_{c}, \mathrm{~S}_{6}^{\mathrm{R} 1}$ disappears and we observe a periodic orbit in place of the heteroclinic cycle. The period of this orbit tends to infinity, as $T a$ approaches the point of bifurcation $T a_{c}$.

Kinetic and magnetic energies for the periodic orbit for $T a=506$, which is close to the point of the saddle-node bifurcation (hence the period of the orbit is large) is shown on Fig. 20. The minima of magnetic energy are attained when the trajectory is close to $R_{1}$; from these steady states it jumps rapidly to the former $\mathrm{S}_{6}^{\mathrm{R} 1}$ (kinetic energy becoming close to 161 ), from where it slowly moves back towards $\mathrm{R}_{1}$.

Notably, close to the critical Ta we observe not a periodic, but rather a chaotic behaviour (see Fig. 20). The non-periodicity (the behaviour is, loosely speaking, periodic, but "periods" significantly vary) can be caused by reasons of numerical nature, such as round-off errors or other numerical noise accumulating during the long periods. As noticed by Busse and Heikes (1980), round-off errors are comparable with the amplitude of the integrated field near stagnation point, significantly affecting trajectories approaching a heteroclinic cycle: Numerical noise can result in emergence of quasi-periodic regimes with randomly varying "periods" (Stone and Holmes, 1990), like we observe here, as well as strictly periodic cycles of a large period (Nore et al., 2003), or alter the amplitude of cycles (Stone and Armbruster, 1999). Numerical noise can be expected to affect similarly large-period orbits near the critical parameter value.

\subsection{MHD attractors emerging from $\mathrm{R}_{1}, 506<T a \leq 718$}

The primary steady state $\mathrm{S}_{6}^{\mathrm{R} 1}$, connected with the $\mathrm{S}_{5}^{\mathrm{R} 1}$ branch by the periodic orbit $\mathrm{P}_{3}^{\mathrm{R} 1}$, is different from $S_{5}^{\mathrm{R} 1}$ and other $\mathrm{S}^{\mathrm{R} 1}$ steady states considered above in that it is related not to the dominant but a subdominant magnetic mode of $R_{1}$. The symmetry group of the steady state $\mathrm{S}_{6}^{\mathrm{R} 1}$ is not a subgroup of the symmetry group of $\mathrm{S}_{2}^{\mathrm{R} 1}$ (coinciding with the symmetry group of the dominant magnetic mode) and it has a twice smaller period in the direction along the axis of rolls, indicating that $\mathrm{S}_{6}^{\mathrm{R} 1}$ is unrelated to the dominant magnetic mode (cf. Fig. 5 (c) and Fig. 11 (f)). We have computed the subdominant magnetic mode by 

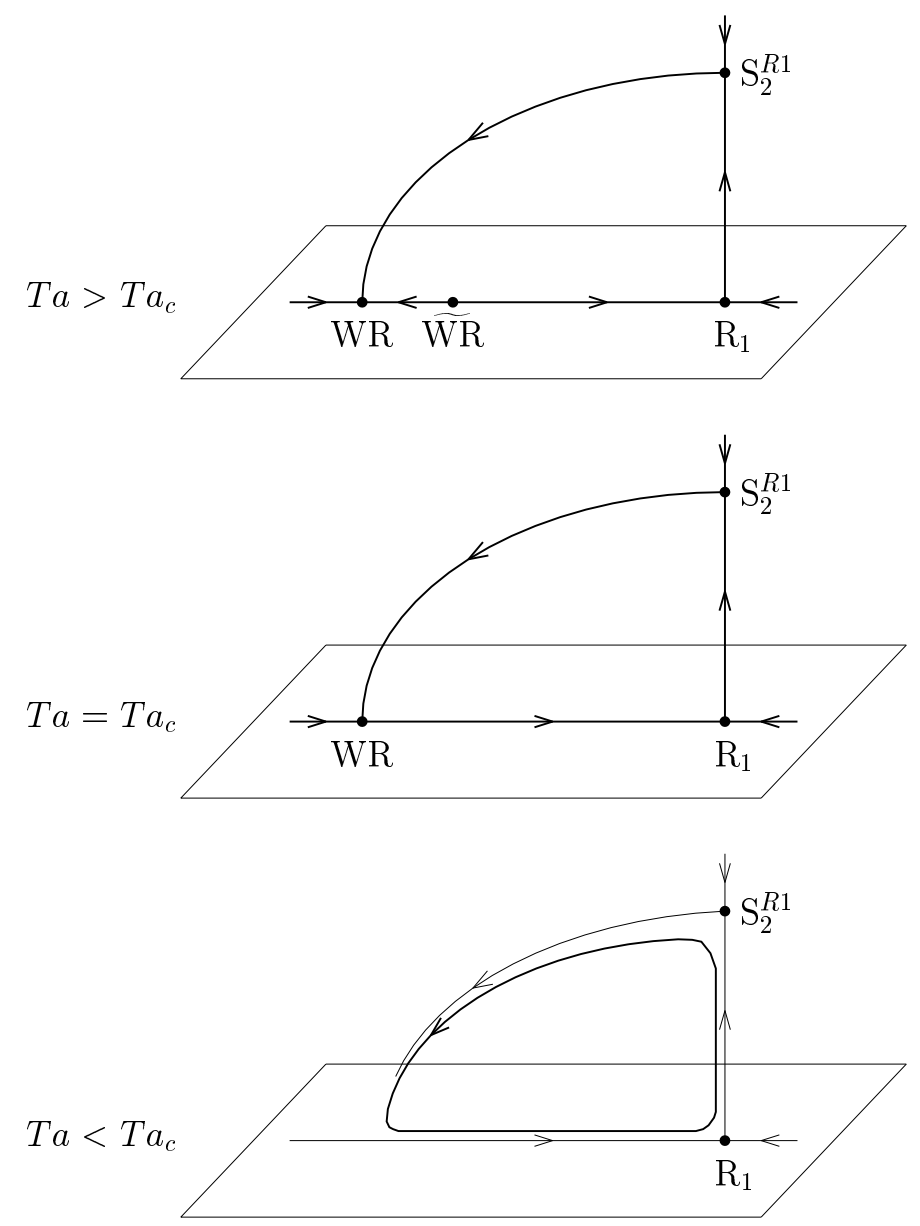

Figure 21: Phase portrait of the MHD system near $T a=T a_{c} \approx 718.16$.

restricting the kinematic dynamo problem for $\mathrm{R}_{1}$ on the subspace $\operatorname{Fix}\left(q \gamma_{L / 4}^{y}\right)$. The subdominant magnetic mode, which is dominant in $\operatorname{Fix}\left(q \gamma_{L / 4}^{y}\right)$, has the same group of symmetries as that of $\mathrm{S}_{6}^{\mathrm{R} 1}$ and a spatial structure similar to that of the magnetic field of $\mathrm{S}_{6}^{\mathrm{R} 1}$ (see Fig. 111(f)). The associated eigenvalue is real and positive in the interval $509 \leq T a \leq 682$ and admits the maximum 0.4 at $T a=598$. On increasing $T a, \mathrm{~S}_{6}^{\mathrm{R} 1}$ becomes unstable in a pitchfork bifurcation with emergence of the steady state $\mathrm{S}_{7}^{\mathrm{R} 1}$, becoming unstable in its turn in a subcritical pitchfork bifurcation at $T a=682.26$.

The symmetry group of $\mathrm{S}_{8}^{\mathrm{R} 1}$ is a subgroup of the symmetry group of $\mathrm{S}_{2}^{\mathrm{R} 1}$, indicating that the former state has possibly bifurcated from the latter one. On decreasing $T a, \mathrm{~S}_{8}^{\mathrm{R} 1}$ becomes unstable in a subcritical pitchfork bifurcation; in the interval $674 \leq T a \leq 682$ the steady state coexists with $\mathrm{S}_{7}^{\mathrm{R} 1}$. When $T a$ is increased, a stable periodic orbit $\mathrm{P}_{4}^{\mathrm{R} 1}$ emanates in a Hopf bifurcation from $\mathrm{S}_{8}^{\mathrm{R} 1}$. On increasing $T a$ further, this periodic orbit terminates on a structurally unstable heteroclinic cycle. We discuss this bifurcation in detail in the following subsection.

\subsection{MHD attractors emerging from $\mathrm{R}_{1}$, Ta near 718}

We have discussed in subsection 4.3 a periodic orbit terminating on a structurally unstable heteroclinic cycle involving connections between two steady states. At $T a=T a_{c} \approx 718.16$ a similar bifurcation takes place. The periodic orbit $\mathrm{P}_{4}^{\mathrm{R} 1}$ terminates on a structurally unstable heteroclinic cycle with three steady states involved, when one of the steady states undergoes a saddle-node bifurcation. 
A sketch of geometry of the phase space is shown on Fig. 21, The plane represents the hydrodynamic subspace invariant under the symmetry $q$, and the vertical direction the complementary magnetic subspace. We describe the bifurcation starting from the larger values of $T a$ slightly above the point $T a=T a_{c}$ of the saddle-node bifurcation of WR. For such $T a$, in the hydrodynamic subspace we observe $\mathrm{R}_{1}$, stable in the hydrodynamic subspace, stable WR and its unstable counterpart, WR. A part of the one-dimensional unstable manifold of $\tilde{W R}$ terminates on $R_{1}$, the other one on WR (since $\tilde{W R}$ emerges in a subcritical bifurcation from $R_{1}$ and it disappears in a saddle-node collision with WR). Connections from $\tilde{W R}_{\mathrm{R}}$ to $\mathrm{R}_{1}$ and WR are structurally stable, because $R_{1}$ and $W R$ are stable in the hydrodynamic subspace. The connection from $\mathrm{R}_{1}$ to $\mathrm{S}_{2}^{\mathrm{R} 1}$ belongs to $\operatorname{Fix}\left(q \gamma_{L / 2}^{y}\right)$ and it is structurally stable, because $\mathrm{S}_{2}^{\mathrm{R} 1}$ is stable in this subspace (this has been checked numerically). The unstable manifold of $\mathrm{S}_{2}^{\mathrm{R} 1}$ belongs to $\mathrm{Fix}\left(s_{2}\right)$ and it terminates on WR, stable in this subspace.

At $T a=T a_{c}, \mathrm{WR}$ and $\tilde{\mathrm{WR}}$ collide creating the heteroclinic cycle $\mathrm{WR} \rightarrow \mathrm{R}_{1} \rightarrow \mathrm{S}_{2}^{\mathrm{R} 1} \rightarrow \mathrm{WR}$ in the subspace $\operatorname{Fix}\left(s_{2}\right)$. The cycle is asymptotically stable within this subspace, but not in the entire phase space, because WR possesses a growing magnetic mode. The cycle is structurally unstable; it exists only for $T a=T a_{c}$. For $T a<T a_{c}$, WR disappears and we observe a periodic orbit in place of the heteroclinic cycle. The period of the orbit tends to infinity, as $T a$ approaches the point of bifurcation. Surprisingly, the orbit is asymptotically stable, despite it has bifurcated from an asymptotically unstable heteroclinic cycle.

We illustrate the behaviour described above by plots of kinetic and magnetic energies and discrepancies for the symmetries $s_{2}$ and $q \gamma_{L / 2}^{y}$ for a periodic orbit at $T a=717.9$ (Fig. 22), near the point of bifurcation, where the orbit is similar to the structurally unstable heteroclinic cycle. Plateaux of constant values of kinetic energy on Fig. 22 (such as $1870<t<1930$ and $1990<t<2060$ ) represent time intervals when the trajectory is near the steady states $\mathrm{R}_{1}$ and $\mathrm{S}_{2}^{\mathrm{R} 1}$, respectively. The inflection point near $t=2090$ shows where the trajectory is in the vicinity of the former steady state WR. Magnetic energy is small during the transition from the former $\mathrm{WR}$ to $\mathrm{R}_{1}$, attaining a minimum at $t=2110$. The symmetry $q \gamma_{L / 2}^{y}$ is present during the transition from $R_{1}$ to $S_{2}^{R 1}$. An exponential growth of the symmetry discrepancy takes place during the departure from $\mathrm{S}_{2}^{\mathrm{R} 1}$. The symmetry $s_{2}$ is slightly broken near the former WR.

The discrepancy of the symmetry $s_{2}$ is shown for an axis changing position in time (the position is optimised to minimise the discrepancy). The $y$ coordinate of the moving axis is plotted on Fig. 22 (c); the displacement in the $x$ direction is by several orders of magnitude smaller, and it is described by a function of a similar shape. The motion of the axis can be regarded as a drift of the cycle $\mathrm{P}_{3}^{\mathrm{R} 1}$ along a group orbit. In accordance with the theory of Krupa (1990), the axis is steady when the symmetry $s_{2}$ is present; it moves, when the discrepancy is maximal (for instance, at the time interval $2140<t<2160$ ).

For $\mathrm{Ta}=719$ a trajectory starting near $\mathrm{S}_{2}^{\mathrm{R} 1}$ visits the following steady states: $\mathrm{S}_{2}^{\mathrm{R} 1} \rightarrow \mathrm{WR} \rightarrow \mathrm{R}_{\mathrm{D}} \rightarrow$ $\mathrm{S}_{3}^{\mathrm{RD}} \rightarrow \mathrm{S}_{2}^{\mathrm{RD}}$.

\subsection{MHD attractors emerging from WR, $721 \leq T a \leq 725$}

The primary steady state $\mathrm{S}^{\mathrm{WR}}$ emerges from WR in a pitchfork bifurcation at $T a \approx 725.71$, when the magnetic growth rate becomes positive on decreasing $T a$. It is stable in a short interval of $T a$ and disappears in a saddle-node bifurcation at $T a \approx 720.25$.

\subsection{MHD attractors emerging from $\mathrm{R}_{\mathrm{D}}, 685 \leq T a \leq 787$}

We have found numerically that $R_{D}$ possesses two types of dominant magnetic modes (see Table 2 and Fig. 3). In the interval of $T a$ where $R_{D}$ exists there are two windows of kinematic dynamo action, resulting in two intervals of nonlinear dynamos; the lower one is $684 \leq T a \leq 787$.

The first (on increasing $T a$ ) attractor $\mathrm{S}_{1}^{\mathrm{RD}}$ is primary, but it is related to a subdominant magnetic mode. The mode has the same spatial structure as the respective nonlinear steady state displayed on Fig. 11 (k). The mode has the period $L / 3$ along the axes of rolls (in contrast with periods $L / 2$ and $L$ for two other magnetic modes, Fig. $11(\mathrm{~m})$ and $(\mathrm{n}))$. The associated eigenvalue is real and positive for $685 \leq T a \leq 711$, the maximum is 0.1 . 
(a)

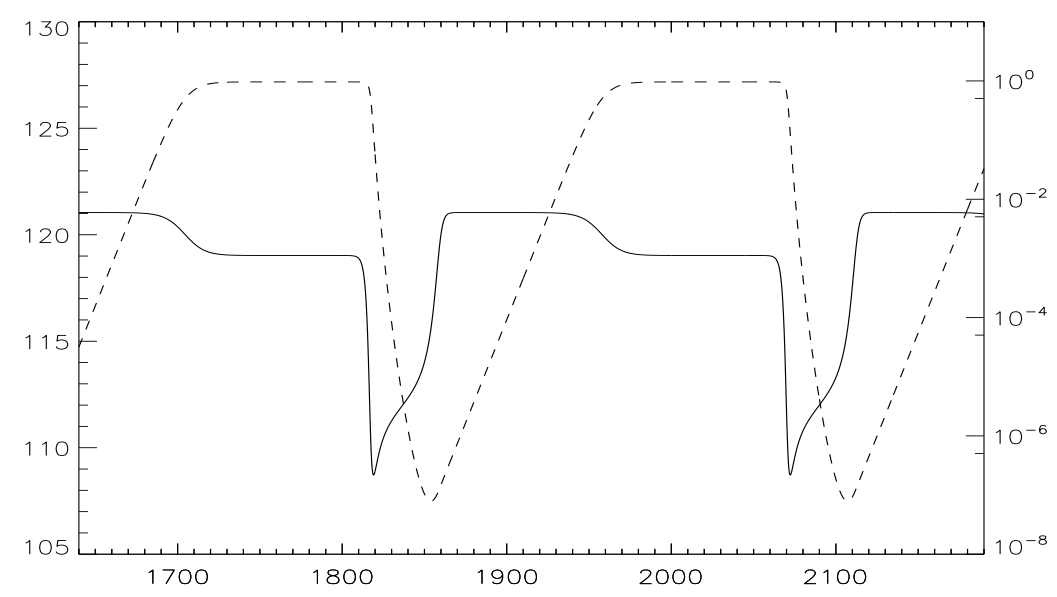

(b)

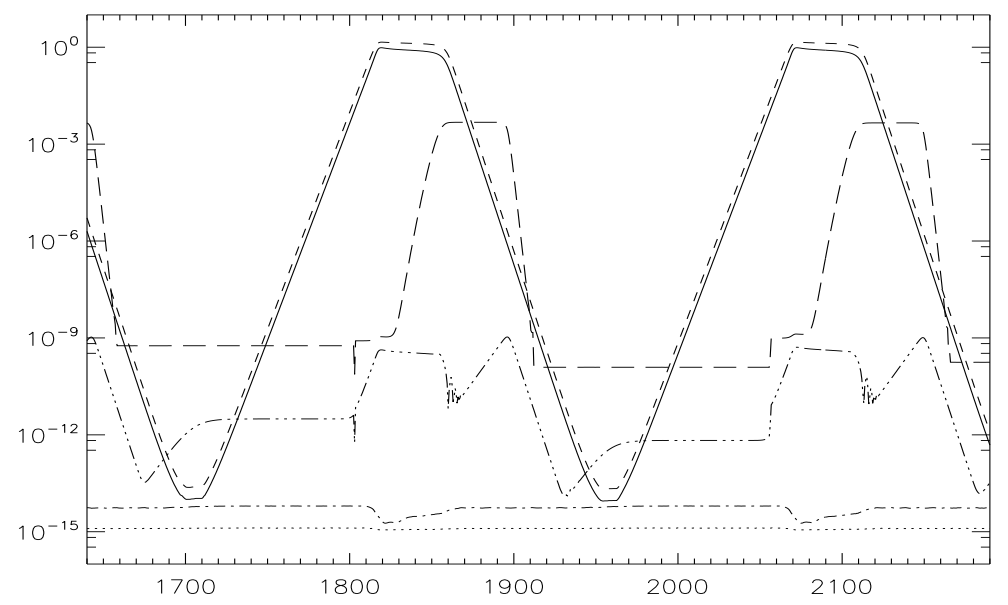

(c)

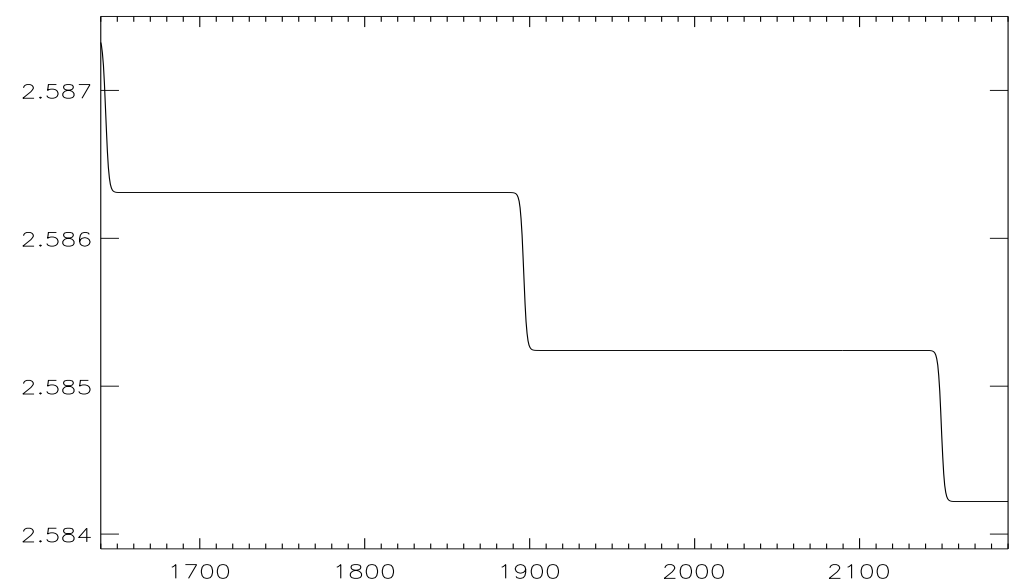

Figure 22: Kinetic (solid line) and magnetic (dashed line) energies (a), symmetry discrepancies (b) and the $y$ coordinate of the axis of the symmetry $s_{2}$ (c) versus time (horizontal axis) for $T a=717.9, \mathrm{P}_{4}^{\mathrm{R} 1}$. On (b), the following symmetry discrepancies are traced: in the flow, $\gamma_{L / 2}^{y}$ (solid line), $r \gamma_{L / 2}^{x}$ (dotted line), $s_{2}$ (dash-and-three-dots line); in magnetic field: $\gamma_{L / 2}^{y} q$ (short-dash line), $r \gamma_{L / 2}^{x}$ (dash-and-dot line), $s_{2}$ (long-dash line). Discrepancy of a symmetry $s$ in a field $\mathbf{f}$ is measured as $\sqrt{\int|\mathbf{f}-s(\mathbf{f})|^{2} d \mathbf{x} / \int|\mathbf{f}|^{2} d \mathbf{x}}$, where integration over a periodicity cell is assumed. 
Another primary MHD attractor is $\mathrm{S}_{3}^{\mathrm{RD}}$ bifurcating from $\mathrm{RD}$ in a pitchfork bifurcation when the respective eigenvalue of the kinematic dynamo problem crosses the imaginary axis. The two branches of primary steady states are connected by the branch $\mathrm{S}_{2}^{\mathrm{RD}}$, with a smaller symmetry group (cf. Fig. 11] (k), (l), (m)).

\subsection{MHD attractors emerging from $\mathrm{R}_{\mathrm{D}}, 1118 \leq T a \leq 1355$}

The third primary branch emanating from $\mathrm{R}_{\mathrm{D}}$, the steady state $\mathrm{S}_{4}^{\mathrm{RD}}$, is always stable when exists. Both ends of the branch terminate on $\mathrm{R}_{\mathrm{D}}$ at $T a=1117.47$ and $T a=1355.20$.

\section{Conclusion}

Our results demonstrate that the influence of rotation on magnetic field generation by thermal convection is non-monotonic and in no way simple. Its nature can only be understood by a careful investigation of attractors of the underlying dynamical system and bifurcations of branches of convective MHD regimes. Such an investigation is made more exciting by the presence of a large group of symmetries of the dynamical system. Even close to the critical point for the onset of convective motion, in the terms of the Taylor number, the bifurcation diagram is quite complex. There are several intervals of coexistence of two or three convective MHD attractors (also sometimes with a non-generating hydrodynamic one).

An overall picture that we have obtained fits well the usual beliefs, that up to a point an increase of the rate of rotation from zero benefits magnetic field generation both in linear and nonlinear regimes, and after attaining a maximum of the mean magnetic energy (in our simulations, $E_{m}=32.30$ in the regime $\mathrm{S}_{2}^{\mathrm{R} 1}$ for $T a=216$ ) on a further increase of the rotation rate $E_{m}$ gradually falls off till magnetic field generation ceases, and at still higher angular velocities the fluid flow is arrested. However, the presence of many individual branches and windows of nonlinear dynamo action (e.g., between the MHD steady states $\mathrm{S}_{3}^{\mathrm{RD}}$ and $\mathrm{S}_{4}^{\mathrm{RD}}$ ) adds a rich "small-scale structure" to this otherwise "smooth" general picture, on some intervals of $T a$ even reversing it. For instance, an increase of the rate of rotation from zero inhibits magnetic field generation in the periodic regime $\mathrm{P}^{\mathrm{TW}}$, rather than enhances it.

Apparently the conjecture, that increasing the Taylor number first helps, afterwards hinders and finally halts magnetic field generation, has not yet been proved, but it appears to be correct for all parameter values (provided the Rayleigh number is sufficiently large). In the absence of magnetic field the critical Rayleigh number for the onset of convection grows with the Taylor number (see Chandrasekhar, 1961); thus, in the hydrodynamic case fast rotation is guaranteed to arrest convective flows. If a magnetic field is present, the system is more complex and various phenomena - such as subcritical loss of stability of the basic state with the fluid at rest - are not ruled out for sufficiently strong initial magnetic fields; this can result in extension of the interval of the Taylor number, for which convective MHD states persist for the given other parameter values. However, it is unlikely that this interval becomes infinite even for $P_{m} \gg P$, since - in physical terms - magnetic field generation relies on advection by the fluid, and not vice versa. This question awaits further investigation.

We have observed a number of interesting bifurcations. To the best of our knowledge, two global bifurcations were not observed before. They are similar to the SNIC (saddle-node on invariant circle) bifurcation, and their peculiarity stems from the presence of a non-trivial symmetry group in the convective MHD system. In these bifurcations, at $T a \approx 506.07$ and $T a \approx 718.16$, a periodic orbit terminates not on a homoclinic (as in the SNIC), but a heteroclinic cycle, whose existence relies on the presence of the symmetry group. Among more common, albeit rather seldom observed in natural systems families, that we have encountered, an incomplete Feigenbaum sequence of "period doubling bifurcations of a torus", $\mathrm{F}^{\mathrm{TW}}$ (occurring between $T a=57$ and 80 ) is notable, as well as an intermittent sequence of chaotic and quasiperiodic regimes, $\mathrm{C}^{\mathrm{SW}}$ (occurring for $78 \leq T a \leq 126$ ), in which existence of the quasiperiodic regimes can be apparently linked with frequency locking.

Another unusual set of bifurcations observed on the interval $216<T a<224$ is associated with a pair of complex eigenvalues of the linearisation of the dynamical system, which are simultaneously vanishing. Numerical examination of eigenvalues of the supposed trivial steady state $\mathrm{S}_{2}^{\mathrm{R} 1}$ suggests that the observed bifurcations in our convective MHD system are not linked with a Takens-Bogdanov bifurcation occurring 
for nearby parameter values, but we do not rule out a finite perturbation of a bifurcation of this type. We plan to perform a further investigation of the bifurcation in order to gain a mathematical understanding of its nature.

We have found a number of branches of MHD steady states, which are parity-invariant (i.e. have the symmetry $\left.r s_{2}\right)$ or possessing the symmetry about a vertical axis, $s_{2}$. Certain periodic orbits $(T a=57$, the family $\mathrm{F}^{\mathrm{TW}} ; \mathrm{Ta}=123$, the family $\mathrm{C}^{\mathrm{SW}} ; 216.13 \leq T a \leq 223.3$, branch $\mathrm{P}_{1}^{\mathrm{R} 1}$ ) are not pointwise symmetric, but symmetric on average, i.e. the symmetry with a time shift by a half of temporal period is present. Possession of such a symmetry is an important property of attractors, since in convective MHD systems with this symmetry the global $\alpha$-effect is zero. Whilst in the presence of the global $\alpha$-effect the system is inherently unstable to large-scale perturbations, in its absence the instability, when present, develops on time scales of a larger order. In the latter case it is described by a highly complex nonlinear mixed system of PDE's of the second and third order (see Zheligovsky, 2009b), incorporating such physical effects, as combined eddy diffusivity and eddy advection. We are planning to follow this line of research.

For the chosen values of the Rayleigh and magnetic Prandtl numbers, which are not particularly high, the convective system is not far from the onset of convection and magnetic field generation, and the collection of hydrodynamic and magnetic structures that we encounter is not rich (in the MHD regimes the flows take the form of perturbed rolls, and magnetic field concentrates in half-ropes located near the boundaries). More complex structures are produced in a more vigorous convection for higher values of the Rayleigh and the magnetic Prandtl numbers.

A natural extension of our investigation consists of continuation of branches of regimes, that we have found, in other parameters, which would significantly contribute for gaining an insight into the mathematics and physics of convective dynamos (i.e., of the geometry of branches of attractors in the parameter space). This project is, obviously, quite resource demanding, but in our opinion this approach is more promising for the deeper understanding of convective MHD systems, than analysis of individual runs for extreme parameter values.

\section{Acknowledgements}

RC is supported by "Fundação para a Ciência e a Tecnologia" (Portugal), grant SFRH/BD/23161/2005. Part of this research was carried out during the visits of OP and VZ to the School of Engineering, Computer Science and Mathematics, University of Exeter (UK) in January - April 2008. OP and VZ are grateful to the Royal Society for their financial support. Research visits of OP and VZ to Observatoire de la Côte d'Azur (France) in the autumns of 2007 and 2008 were supported by the French Ministry of Education. OP and VZ are partially supported by the grants ANR-07-BLAN-0235 OTARIE from Agence nationale de la recherche (France) and 07-01-92217-CNRSL_a from the Russian foundation for basic research. Computations were carried out on the computer "Mésocentre SIGAMM (Simulations Interactives et Visualisation en Géophysique, Astronomie, Mathématique et Mécanique)" hosted by Observatoire de la Côte d'Azur, and on the supercomputer MILIPEIA at the Laboratory for Advanced Computing at the University of Coimbra (Portugal).

\section{References}

[1] A.P. Bassom, K. Zhang. Strongly nonlinear convection cells in a rapidly rotating fluid layer. Geophys. Astrophys. Fluid Dyn. 76 (1994) 223-238.

[2] J.P. Boyd. Chebyshev and Fourier spectral methods. Dover (2001).

[3] A. Brandenburg, K. Subramanian. Astrophysical magnetic fields and nonlinear dynamo theory. Physics Reports 417 1-209 (2005).

[4] F.H. Busse. Homogeneous dynamos in planetary cores and in the laboratory. Ann. Rev. Fluid Mech. 32 (2000) $383-408$.

[5] F.H. Busse, K.E. Heikes. Convection in a rotating layer: a simple case of turbulence. Science 208 (1980) 173-175.

[6] F. Busse, E. Dormy, R. Simitev, A. Soward. Dynamics of rotating fluids. In: Mathematical aspects of natural dynamos. E. Dormy, A.M. Soward (eds.) CRC Press, Roca Baton (2007) 119-198.

[7] F. Cattaneo, T. Emonet, N. Weiss. On the interaction between convection and magnetic field. Astrophysical J. 588 (2003) 1183-1198.

[8] F. Cattaneo, D.W. Hughes. Dynamo action in a rotating convective layer. J. Fluid Mech. 553 (2006) $401-418$. 
[9] S. Chandrasekhar. Hydrodynamic and hydromagnetic stability. Oxford Univ. Press (1961).

[10] S. Childress. $\alpha$-effect in flux ropes and sheets. Phys. Earth Planet. Inter. 20 (1979) 172-180.

[11] S. Childress, A.M. Soward. On the rapid generation of magnetic field. In: Chaos in astrophysics. J.R. Buchler (ed.) D. Reidel, Dordrecht (1985) 233-244.

[12] J. Christensen-Dalsgaard, M.J. Thompson. Observational results and issues concerning the tachocline. In: The solar tachocline. D.W. Hughes, R. Rosner, N.O. Weiss (eds.) Cambridge Univ. Press (2007) 53-85.

[13] P. Coullet. Stability of the scenarios toward chaos. In: Chaos and statistical methods. Y. Kuramoto (ed.) Springer-Verlag, Berlin (1984) 62-71.

[14] A. Demircan, N. Seehafer. Dynamo in asymmetric square convection. Geophys. Astrophys. Fluid Dyn. 96 (2002) $461-479$.

[15] Mathematical aspects of natural dynamos. E. Dormy, A.M. Soward (eds.) CRC Press, Roca Baton (2007).

[16] M.J. Feigenbaum. Quantitative universality for a class of nonlinear transformations. J. Stat. Phys. 19 (1978) $25-52$.

[17] D.J. Galloway, V.A. Zheligovsky. On a class of non-axisymmetric flux rope solutions to the electromagnetic induction equation. Geophys. Astrophys. Fluid Dyn. 76 (1994) 253-264.

[18] S.Ya. Gertsenshtein, V.A. Zheligovsky, O.M. Podvigina, R.A. Chertovskikh. Generation of a magnetic field by threedimensional convective flows of a conducting fluid in the rotating horizontal layer. Doklady Physics 52 (2007) 653-655.

[19] S.Ya. Gertsenshtein, R.A. Chertovskikh. Generation of a magnetic field by convective flows in a rotating horizontal layer. Fluid Dynamics 43 (2008) 248-256.

[20] A.V. Getling. Rayleigh-Bénard convection: structures and dynamics. World Scientific Publ., Singapore (1998).

[21] G.A. Glatzmaier, P.H. Roberts. A three-dimensional convective dynamo solution with rotating and finitely conducting inner core and mantle, Phys. Earth Planet. Inter. 91 (1995) 63-75.

[22] M. Golubitsky, I.N. Stewart, D. Schaeffer. Singularities and Groups in Bifurcation Theory. Volume 2. Appl. Math. Sci., vol. 69. Springer-Verlag, New York (1988).

[23] E. Grote, F.H. Busse. Dynamics of convection and dynamos in rotating spherical fluid shells. Fluid Dyn. Res. 28 (2001) 349-368.

[24] J. Guckenheimer, P. Holmes. Structurally stable heteroclinic cycles. Math. Proc. Cambridge Phil. Soc. 103 (1988) $189-192$.

[25] The solar tachocline. D.W. Hughes, R. Rosner, N.O. Weiss (eds.) Cambridge Univ. Press (2007).

[26] N. Ishinara, S. Kida. Dynamo mechanism in a rotating spherical shell: competition between magnetic field and convection vortices. J. Fluid Mech. 465 (2002) 1-32.

[27] E.M. Izhikevich. Dynamical systems in neuroscience: the geometry of excitability and bursting. MIT Press (2006).

[28] M. Krupa. Bifurcations in relative equilibria. SIAM J. Math. Anal. 21 (1990) 1453-1486.

[29] P.C. Matthews. Dynamo action in simple convective flows. Proc. R. Soc. 455 (1999) 1829-1840.

[30] M. Meneguzzi, A. Pouquet. Turbulent dynamos driven by convection. J. Fluid Mech. 205 (1989) 297-318.

[31] H.K. Moffatt. Magnetic field generation in electrically conducting fluids. Cambridge Univ. Press (1978).

[32] C. Nore, L.S. Tuckerman, O. Daube S. Xin. The 1:2 mode interaction in exactly counter-rotating von Kármán swirling flow. J. Fluid Mech. 477 (2003) 51-88.

[33] E. Ott. Chaos in dynamical systems. Cambridge Univ. Press (2002).

[34] E.N. Parker. Cosmical magnetic fields: Their origin and their activity. Clarendon Press (1979).

[35] R. Peyret. Spectral methods for incompressible viscous flow. Springer Verlag, Berlin (2002).

[36] E.R. Priest. Solar magnetohydrodynamics. D. Reidel, Dordrecht (1984).

[37] O.M. Podvigina. Magnetic field generation by convective flows in a plane layer. Eur. Phys. J. B 50 (2006) 639-652

[38] O.M. Podvigina. Magnetic field generation by convective flows in a plane layer: the dependence on the Prandtl number. Geophys. Astrophys. Fluid Dyn. 102 (2008) 409-433.

[39] O.M. Podvigina, P. Ashwin. Heteroclinic cycles in the $1: \sqrt{2}$ mode interaction with applications to Boussinesq convection. Physica D 234 (2007) 23-48.

[40] B. von Rekowski, W. Dobler, A. Shukurov, A. Brandenburg. Two-dimensional disk dynamos with vertical outflows into a halo. In: Dynamo and dynamics: a mathematical challenge. P. Chossat, D. Armbruster, I. Oprea (eds.) Kluwer, Dordrecht (2001) 305-312.

[41] P.H. Roberts, G.A. Glatzmaier. Geodynamo theory and simulations. Rev. Mod. Phys. 72 (2000) 1081-1123.

[42] D. Ruelle, F. Takens. On the nature of turbulence. Commun. Math. Phys. 20 (1971) 167-192.

[43] A.A. Ruzmaikin, A.M. Shukurov, D.D. Sokoloff. Magnetic fields of galaxies. Kluwer, Dordrecht (1988).

[44] A. Shukurov, D. Sokoloff. Astrophysical dynamos. In: Dynamos: Lecture notes of the Les Houches summer school 2007. Ph. Cardin, L.F. Cugliandolo (eds.) Elsevier (2008) 251-299.

[45] Fluid Dynamics and dynamos in astrophysics and geophysics. A.M. Soward, C.A. Jones, D.W. Hughes, N.O. Weiss (eds.) CRC Press, London (2005).

[46] E.J. Spence, K. Reuter, C.B. Forest. A spherical plasma dynamo experiment. Astrophys. J. 700 (2009) 470-478.

[47] M.G. St Pierre. The strong field branch of the Childress-Soward dynamo. In: Solar and planetary dynamos. M.R.E. Proctor, P.C. Matthews, A.M. Rucklidge (eds.) Cambridge Univ. Press (1993) 295-302.

[48] E. Stone, D. Armbruster. Noise and O(1) amplitude effects on heteroclinic cycles. Chaos. 9 (1999) 499-506.

[49] E. Stone, P. Holmes. Random perturbations of heteroclinic attractors. SIAM J. Appl. Math. 50 (1990) 726-743.

[50] F. Takahashi, M. Matsushima. Dynamo action in rotating spherical shell at high Rayleigh number. Phys. Fluids 17 (2005) 076601.

[51] S.M. Tobias, N.O. Weiss. The solar dynamo and the tachocline. In: The solar tachocline. D.W. Hughes, R. Rosner, N.O. Weiss (eds.) Cambridge Univ. Press (2007) 319-350.

[52] Ya.B. Zeldovich. The magnetic field in the two-dimensional motion of a conducting turbulent fluid. Journ. Exper. Theor. Phys. 31 (1956) 154-156; Engl. transl.: Sov. Phys. J.E.T.P. 4 (1957) 460-462. 
[53] V. Zheligovsky. Numerical solution of the kinematic dynamo problem for Beltrami flows in a sphere. J. Scient. Computing 8 (1993a) 41-68.

[54] V.A. Zheligovsky. A kinematic magnetic dynamo sustained by a Beltrami flow in a sphere. Geophys. Astrophys. Fluid Dyn. 73 (1993b) 217-254.

[55] V. Zheligovsky. Determination of a flow generating a neutral magnetic mode. Phys. Rev. E 80 (2009a) 036310 http://arxiv.org/pdf/0906.5431v1.

[56] V.A. Zheligovsky. Amplitude equations for weakly nonlinear two-scale perturbations of free hydromagnetic convective regimes in a rotating layer. Geophys. Astrophys. Fluid Dyn. 103 (2009b) 397-420 http://arxiv.org/abs/0809.1195v2].

[57] V.A. Zheligovsky. Generation of a symmetric magnetic field by thermal convection in a plane rotating layer. Magnetohydrodynamics, 46 (2010) 3-22 http://arxiv.org/abs/0906.5380v2]. 